PREPARED FOR THE U.S. DEPARTMENT OF ENERGY, UNDER CONTRACT DE-AC02-76CH03073

PPPL-3729

PPPL-3729

UC-70

Monte Carlo Sampling of Negative-temperature Plasma States

by

John A. Krommes and Sharadini Rath

July 2002

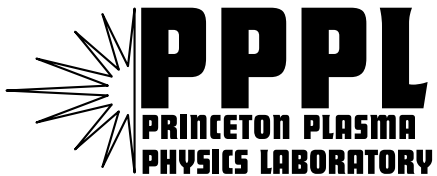

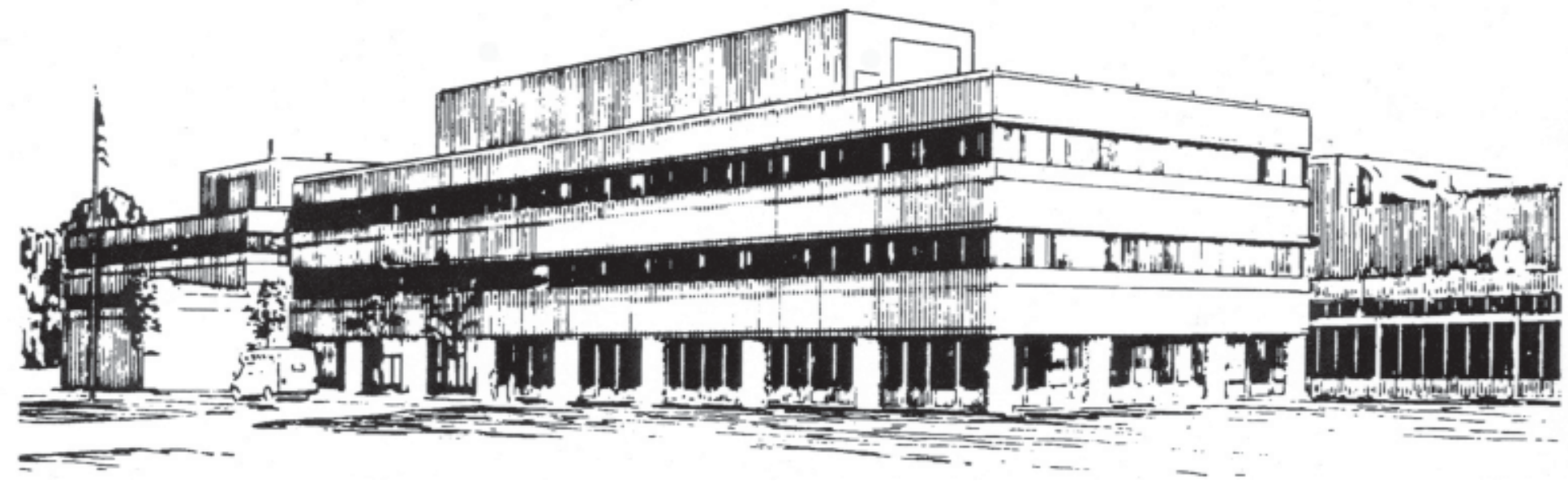

PRINCETON PLASMA PHYSICS LABORATORY PRINCETON UNIVERSITY, PRINCETON, NEW JERSEY 


\section{PPPL Reports Disclaimer}

This report was prepared as an account of work sponsored by an agency of the United States Government. Neither the United States Government nor any agency thereof, nor any of their employees, makes any warranty, express or implied, or assumes any legal liability or responsibility for the accuracy, completeness, or usefulness of any information, apparatus, product, or process disclosed, or represents that its use would not infringe privately owned rights. Reference herein to any specific commercial product, process, or service by trade name, trademark, manufacturer, or otherwise, does not necessarily constitute or imply its endorsement, recommendation, or favoring by the United States Government or any agency thereof. The views and opinions of authors expressed herein do not necessarily state or reflect those of the United States Government or any agency thereof.

\section{Availability}

This report is posted on the U.S. Department of Energy's Princeton Plasma Physics Laboratory Publications and Reports web site in Fiscal Year 2002. The home page for PPPL Reports and Publications is: http://www.pppl.gov/pub_report/

DOE and DOE Contractors can obtain copies of this report from:

U.S. Department of Energy

Office of Scientific and Technical Information

DOE Technical Information Services (DTIS)

P.O. Box 62

Oak Ridge, TN 37831

Telephone: (865) 576-8401

Fax: (865) 576-5728

Email: reports@adonis.osti.gov

This report is available to the general public from:

National Technical Information Service

U.S. Department of Commerce

5285 Port Royal Road

Springfield, VA 22161

Telephone: 1-800-553-6847 or

(703) 605-6000

Fax: (703) 321-8547

Internet: http://www.ntis.gov/ordering.htm 


\title{
Monte Carlo sampling of negative-temperature plasma states
}

\author{
John A. Krommes* and Sharadini Rath ${ }^{\dagger}$ \\ Plasma Physics Laboratory, Princeton University, P.O. Box 451, Princeton, New Jersey 08543
}

(Dated: August 12, 2002)

\begin{abstract}
A Monte Carlo procedure is used to generate $N$-particle configurations compatible with twotemperature canonical equilibria in two dimensions, with particular attention to nonlinear plasma gyrokinetics. An unusual feature of the problem is the importance of a nontrivial probability density function $\mathcal{P}_{0}(\varphi)$, the probability of realizing a set $\{\varphi\}$ of Fourier amplitudes associated with an ensemble of uniformly distributed, independent particles. This quantity arises because the equilibrium distribution is specified in terms of $\{\varphi\}$, whereas the sampling procedure naturally produces particles states $\Gamma ;\{\varphi\}$ and $\Gamma$ are related via a gyrokinetic Poisson equation, highly nonlinear in its dependence on $\Gamma$. Expansion and asymptotic methods are used to calculate $\mathcal{P}_{0}(\varphi)$ analytically; excellent agreement is found between the large- $N$ asymptotic result and a direct numerical calculation. The algorithm is tested by successfully generating a variety of states of both positive and negative temperature, including ones in which either the longest- or shortest-wavelength modes are excited to relatively very large amplitudes.
\end{abstract}

PACS numbers: $52.30 . \mathrm{Gz}, 52.65 . \mathrm{Pp}, 02.50 . \mathrm{Ng}, 05.10 . \mathrm{Ln}$

\section{INTRODUCTION}

In the present paper we will show how to generate $N$ particle configurations compatible with two-temperature canonical equilibria in two dimensions. The method could be used for testing nonlinear gyrokinetic simulations of plasmas; more generally, the problem raises interesting issues involving equilibrium statistical mechanics and asymptotic analysis.

Particle simulations (often called molecular-dynamics experiments in the literature on neutral fluids) are widely used for studying the equilibrium and nonequilibrium behavior of nonlinear systems [1-4]. Such methods are quite natural when the discreteness of the many-body system is important, but particle simulations have also been employed as viable alternatives to more conventional spectral or finite-difference approaches to the integration of partial differential equations in the continuum approximation. This is particularly true in plasma physics [3], where collective nonlinear, neutral-fluid-like phenomena - essentially independent of the details of the microscopic velocity distribution - are often driven by wave-particle interactions strongly dependent on such details. The conservative nature of the Vlasov or closely related gyrokinetic equation [5] is difficult to handle with conventional techniques because phase-space elements can be stretched and otherwise distorted to scales that are arbitrarily small (in the absence of collisions [6]). Particle simulation deals with this situation efficiently by following the particle or gyrocenter trajectories exactly in an electromagnetic field that is coarse-grained to a finite resolution in space.

\footnotetext{
*Electronic address: krommes@princeton.edu ${ }^{\dagger}$ Present address: 20 Akashganga, Ganeshkhind, Pune 411 007, India.
}

Recent interest in plasma physics has focused on nonequilibrium gyrokinetic phenomena [7, 8]. The nonlinear gyrokinetic equation [5], appropriate for lowfrequency, long-wavelength fluctuations, is derivable from the magnetized Vlasov equation by Hamiltonian transformations $[9]^{1}$ that analytically remove the rapid gyromotion in favor of appropriately defined gyrocenters whose characteristic equations of motion are the generalizations of the familiar drift equations to the case of finite ion gyroradius. In the quasineutrality condition that replaces Poisson's equation, a key role is played by the polarization charge density of the ions (see Appendix A), proportional to the $z$ component of the vorticity of the cross-field $\boldsymbol{E} \times \boldsymbol{B}$ velocity. The resulting nonlinear equations display phenomena closely related to the well-known, unusual behavior of two-dimensional (2D) neutral fluids [10]. For example, the HasegawaMima equation [8, 11], an important limit [9] of the gyrokinetic system that is appropriate for adiabatic electrons and vanishing ion temperature, possesses both an energy- and an enstrophy-related invariant and therefore displays all of the interesting phenomena associated with two-parameter canonical (Gibbsian) equilibria [10] of the (finitely truncated) set of Fourier amplitudes of the electrostatic potential $\varphi$ (or ion gyrocenter density, to within a simple wave-number-dependent scale factor). In the presence of dissipation or antidissipation (e.g., when nonadiabatic electron response is reinstated), those invariants are broken, but the tendency of the nonlinear terms to relax fluctuations to thermal equilibrium is still manifested through the dual cascades [12] — of energy $E$, toward the long wavelengths, and of (potential) enstrophy $\Omega$, toward the short wavelengths - that can be ex-

\footnotetext{
1 A review of some of the formal aspects of the derivation of the nonlinear gyrokinetic Poisson system can be found in App. C of Ref. [8].
} 
cited [13] for forcing at intermediate wave numbers.

A standard test in the development of gyrokinetic particle simulations should therefore be to check that the Hasegawa-Mima limit relaxes to the two-temperature canonical spectrum based on the initial values of energy and enstrophy. Somewhat surprisingly, this has not been done, although the analogous exercise is standard for Vlasov codes [3], where one verifies the thermalequilibrium spectrum $\left\langle\delta E^{2}\right\rangle(\boldsymbol{k}) / 8 \pi=\frac{1}{2} T /\left(1+k^{2} \lambda_{D}^{2}\right)$ ( $T$ being the temperature and $\lambda_{D}$ being the Debye length), and has also been performed for simulations of the full gyrokinetic equation with finite $k_{\|}$[14]. (The thermal-equilibrium fluctuation properties of the gyrokinetic system are discussed in Refs. [15-17].) One explanation for this omission involves the difficulty of achieving random initial particle distributions with specified $E$ and $\Omega$. It is, of course, possible to begin with an arbitrary initialization (e.g., particle positions that are completely independent and uniformly distributed, or alternatively a "quiet start" [18]), calculate the associated $E$ and $\Omega$ and the predicted equilibrium spectrum, then check for relaxation toward that equilibrium, and that certainly provides a nontrivial test of the code. However, reliance on just one or two standard initializations and the arbitrariness of any particular initial state imply that one has no systematic way of exploring the extremes of the $E$ $\Omega$ space, including in particular the interesting regimes of negative temperature. For example, it is noteworthy that the simplest random 2D initialization, in which the particle positions are sampled from a homogeneous, correlation-free distribution, has mean energy and enstrophy compatible with a canonical distribution with positive (and equal) temperatures. (We will derive this result in Sec. II C.)

In addition to the gyrokinetic initialization problem, the generation of negative-temperature particle states is interesting in its own right as a nontrivial problem in statistics. Therefore, in this paper we propose ${ }^{2}$ and theoretically analyze a method of generating $2 \mathrm{D}$ particlestate realizations of canonical equilibria for arbitrary values of the invariants (equivalently, for arbitrary temperatures). The (Monte Carlo) procedure employs the Markov-chain algorithm of Metropolis et al. [19, henceforth called $\mathrm{M}(\mathrm{RT})^{2}$ ] originally (and still [20]) used for investigating the thermodynamic properties of dense liquids. Although the present application is essentially straightforward, it does not appear to have been previously used in this particular context. The subtlety in the calculation is that in the present case the random variables that are canonically distributed are the Fourier amplitudes, whereas one desires realizations of particle positions, which are related to the Fourier amplitudes via a nontrivial nonlinear functional dependence. An

\footnotetext{
2 The initial account of this work was given by J. A. Krommes, Bull. Am. Phys. Soc. 37, 1590 (1992).
}

additional complication is that typically there are many more particles than retained Fourier amplitudes, so the relation between the particle states and the potentials is many-to-one. In the standard application to statistical mechanics, on the other hand, the natural variables of the Gibbs distribution are just the particle phase-space coordinates themselves; no functional relation is involved. Thus in conventional $\mathrm{M}(\mathrm{RT})^{2}$ the successive states of the Markov chain are used for the calculation of analytically intractable ensemble averages over a highly non-Gaussian distribution. In the present case the equilibrium spectrum is purely Gaussian in the Fourier amplitudes, so analytical calculations of arbitrary (static) moments of the amplitudes are straightforward; however, the determination of compatible particle realizations is nontrivial.

For notational convenience, we will denote by $\varphi$ both the field $\varphi(\boldsymbol{x})$ and the set of $\mathcal{M}$ retained Fourier amplitudes $\varphi \equiv\left\{\varphi_{\boldsymbol{k}_{\nu}} \mid \nu=1, \ldots, \mathcal{M}\right\}$.

The necessity for dealing simultaneously with two sets of random variables, namely the particle states $\Gamma$ and the Fourier amplitudes $\varphi$, means that a nontrivial transition probability enters the formalism. In standard Monte Carlo algorithms that deal just with particle probabilities, the basic function $T_{0}\left(\Gamma^{\prime} \mid \Gamma\right)$ describes the probability of proposing state $\Gamma^{\prime}$, given initial state $\Gamma$. Usually this function is just a constant. However, in the present application we begin with a particle state $\Gamma$ [to which corresponds the set of Fourier amplitudes $\varphi(\Gamma)]$ and, effectively, propose an amplitude state $\varphi^{\prime}\left(\Gamma^{\prime}\right)$. The relevant transition probability $\mathcal{T}_{0}\left(\varphi^{\prime} \mid \varphi\right)$ can be related to the probability density function $(\mathrm{PDF}) \mathcal{P}_{0}(\varphi)$ of achieving a particular set of Fourier amplitudes by distributing particles uniformly on a lattice; this function depends nontrivially on $\varphi$. We devote considerable effort to calculating $\mathcal{P}_{0}$ asymptotically and to analyzing its role in the appropriately modified Monte Carlo algorithm.

The organization of this paper is as follows. We devote Sec. II to a review of the form and properties of the standard two-temperature canonical equilibria. In Sec. III we review the algorithm of $\mathrm{M}(\mathrm{RT})^{2}$ and describe how to generalize it for application to the initialization problem. In Sec. IV we generate a variety of two-temperature particle states in order to demonstrate the viability of the method. We summarize and discuss our results in Sec. V. Various details are relegated to appendices. In Appendix A we present for completeness a brief derivation of the Hasegawa-Mima equation using gyrokinetic methods. In Appendix B we derive a formula for $\mathcal{P}_{0}(\varphi)$ and present a few exact results. Finally, we devote Appendix $\mathrm{C}$ to a somewhat lengthy presentation of various approximate calculations of $\mathcal{P}_{0}$ valid for a large number of particles.

\section{TWO-TEMPERATURE EQUILIBRIA}

In essence, we are concerned with statistical sampling from a particular, somewhat unusual canonical probabil- 
ity distribution. In this section we provide the necessary background material. Our conventions for Fourier transforms are introduced in Sec. II A (see also Appendix A of Ref. [8]). Then in Sec. II B we introduce the twotemperature equilibria relevant to the Hasegawa-Mima problem and review their properties.

\section{A. Fourier transform conventions}

We work in a $2 \mathrm{D}$ box of sides $L_{x}$ and $L_{y}$, area $V=L_{x} L_{y}$, on which periodic boundary conditions are imposed. (These are the standard boundary conditions for many particle simulations.) The electrostatic potential is resolved on a rectangular lattice of $M_{\text {tot }}=M_{x} \times M_{y}$ points; e.g., $x_{j}=j \Delta x, \Delta x \doteq L_{x} / M_{x}$, $j=0,1, \ldots, M_{x}-1$ (we use the symbol $\doteq$ for definitions). In practice we consider a square box with $L_{x}=L_{y}=L, M_{x}=M_{y}$; however, we sometimes retain the $x$ or $y$ labels for pedagogical purposes. The area of the fundamental cell is $\Delta V=\Delta x \Delta y$. The associated Fourier components obey, e.g., $k_{x}=n_{x} \delta k_{x}$, where $\delta k_{x} \equiv k_{\min } \doteq 2 \pi / L_{x}$. Wave-number magnitudes are denoted as $k \doteq|\boldsymbol{k}|=\left(k_{x}^{2}+k_{y}^{2}\right)^{1 / 2}$. For arbitrary function $A(\boldsymbol{x})$, the fundamental Fourier conventions are

$$
\begin{aligned}
A_{\boldsymbol{k}} & =V^{-1} \int d \boldsymbol{x} e^{-i \boldsymbol{k} \cdot \boldsymbol{x}} A(\boldsymbol{x}), \\
A(\boldsymbol{x}) & =\sum_{\boldsymbol{k}} e^{i \boldsymbol{k} \cdot \boldsymbol{x}} A_{\boldsymbol{k}} .
\end{aligned}
$$

For points $\boldsymbol{x}_{\boldsymbol{j}}$ on the lattice, we interpret

$$
\int d \boldsymbol{x} \ldots \equiv \sum_{j} \Delta V \ldots
$$

and the pair (1) becomes the discrete Fourier transform, with $\boldsymbol{k}_{\boldsymbol{n}} \cdot \boldsymbol{x}_{\boldsymbol{j}}=2 \pi\left(n_{x} j_{x} / M_{x}+n_{y} j_{y} / M_{y}\right)$. We sometimes write $A_{\boldsymbol{k}_{n}} \equiv A_{n}$; one has

$$
A_{-n}=A_{M-n}=A_{n}^{*},
$$

the last equality holding for functions that are real in $\boldsymbol{x}$ space. Although all operations on the lattice are discrete, we often find the integral form of Eq. (2) to be a convenient shorthand.

\section{B. Two-temperature canonical equilibria}

We consider a conservative nonlinear system of coupled Fourier modes $\psi_{\boldsymbol{k}}$. In the derivation of such equations from continuum equations locally nonlinear in $\boldsymbol{x}$ space, the mode coupling arises from the Fourier convolution theorem and thus extends to $\infty$ in $\boldsymbol{k}$ space. We consider instead a system truncated to a finite number $\mathcal{M}$ of Fourier modes. This corresponds to the actual situation in the simulations and is also required theoretically in order that a conventional statistical dynamics can be introduced [10]. Generally the truncation is spherical, $k_{\min } \leq k \leq k_{\max }$, so $\mathcal{M}<M_{\text {tot }}$. It is assumed that under such truncations two constants of the motion, the energy $\widehat{E}$ and the enstrophy $\widehat{\Omega}$, are preserved. The hat denotes a function of the underlying random Fourier amplitudes: $\widehat{E} \equiv \widehat{E}(\psi), \widehat{\Omega} \equiv \widehat{\Omega}(\psi)$, where the braces denote the collection of all retained modes: $\psi \equiv\left\{\psi_{\boldsymbol{k}_{\nu}} \mid \nu=1, \ldots, \mathcal{M}\right\}$. Here $\nu$ denotes an arbitrary labeling of the modes. Functions without hats will denote the ensemble average, e.g., $E \doteq\langle\widehat{E}\rangle$. It is then well known $[8,10,21]$ that the real and imaginary parts of the $\psi_{\boldsymbol{k}}$ (labeled by the subscripts $r$ and $i$, respectively) can be used as independent variables in standard statistical-mechanics arguments that predict relaxation of arbitrary perturbations to realizations drawn from a microcanonical ensemble. In practice, the Gibbs distribution is used more frequently:

$$
P(\psi)=Z^{-1} \exp [-\alpha \widehat{E}(\psi)-\beta \widehat{\Omega}(\psi)],
$$

where $Z$ is the appropriate normalization integral. The parameters $\alpha$ and $\beta$ serve as inverse temperatures for energy and enstrophy, respectively, and are functions of the ensemble-averaged $E$ and $\Omega$. For some ratios of $\Omega / E$, either $\alpha$ or $\beta$ can be negative, as we will review in detail shortly. In states of negative $\alpha$, the longest-wavelength modes are excited to relatively large levels; in states of negative $\beta$, the shortest-wavelength modes are so excited.

In the application to Hasegawa-Mima dynamics, we may choose $\psi$ to be the appropriately dimensionless electrostatic potential $\varphi$. The Hasegawa-Mima equation [11] is briefly rederived for completeness in Appendix A; it is

$$
\left(1-\nabla_{\perp}^{2}\right) \frac{\partial}{\partial t} \varphi(\boldsymbol{x}, t)+V_{*} \frac{\partial \varphi}{\partial y}+\boldsymbol{V}_{E} \cdot \nabla\left(-\nabla_{\perp}^{2} \varphi\right)=0,
$$

where $V_{*}$ is the diamagnetic drift velocity (considered to be a constant in this approximation), $\boldsymbol{V}_{E} \doteq c \boldsymbol{E} \times$ $\widehat{\boldsymbol{z}} / B, \boldsymbol{E} \doteq-\boldsymbol{\nabla} \varphi$, and the perpendicular direction is with respect to the constant magnetic field $\boldsymbol{B}=B \widehat{\boldsymbol{z}}$. Upon Fourier transformation in space, Eq. (5) becomes

$$
\begin{aligned}
(1+ & \left.k^{2}\right) \frac{\partial \varphi_{\boldsymbol{k}}}{\partial t}+i \omega_{*}\left(k_{y}\right) \varphi_{\boldsymbol{k}} \\
& =\frac{1}{2} \sum_{\boldsymbol{k}+\boldsymbol{p}+\boldsymbol{q}=\mathbf{0}} \widehat{\boldsymbol{z}} \cdot(\boldsymbol{p} \times \boldsymbol{q})\left(\frac{\chi_{\boldsymbol{q}}^{*}-\chi_{\boldsymbol{p}}^{*}}{1+\chi_{\boldsymbol{k}}}\right) \varphi_{\boldsymbol{p}}^{*} \varphi_{\boldsymbol{q}}^{*}
\end{aligned}
$$

where $\boldsymbol{k} \equiv \boldsymbol{k}_{\perp}, \omega_{*}\left(k_{y}\right) \doteq k_{y} V_{*}$, and $\chi_{\boldsymbol{k}} \doteq k_{\perp}^{2}$. This equation is conservative. The more physically relevant model, in which both $\omega_{*}$ and $\chi$ are replaced by complex quantities including dissipative effects, is called the Terry-Horton equation [22, 23]. See Sec. V for further remarks on that equation.

The quadratic invariants of the Hasegawa-Mima equation are

$$
\left(\begin{array}{l}
\widehat{E}(\varphi) \\
\widehat{\Omega}(\varphi)
\end{array}\right)=\frac{1}{2} \sum_{\boldsymbol{k}}\left(\begin{array}{c}
1 \\
k^{2}
\end{array}\right) \widehat{E}_{\boldsymbol{k}}
$$


where

$$
\widehat{E}_{\boldsymbol{k}} \doteq\left(1+k^{2}\right)\left|\widehat{\Phi}_{\boldsymbol{k}}\right|^{2} .
$$

The term 1 in the factor $\left(1+k^{2}\right)$ describes the adiabatic response of the electrons, which stream rapidly along the magnetic field lines and tend to short out charge fluctuations. If that term is ignored (equivalently, if one considers the short-wavelength limit ${ }^{3}$ ), the resulting equation is formally identical to the two-dimensional Navier-Stokes equation, with $\nabla_{\perp}^{2} \varphi$ playing the role of the $z$ component $\omega_{z}$ of the vorticity.

The quantities (7) are invariant when $\boldsymbol{k}$ is summed over all modes out to $\infty$. They remain invariant if one removes from the convolution sum in Eq. (6) all triad interactions with the magnitude of any leg larger than some $k_{\max }$; that corresponds to the spherical truncation mentioned earlier. Later we will consider other truncations and/or weightings. All of those can be embraced by introducing a non-negative weight function $w_{\boldsymbol{k}}$ and generalizing Eq. (7) to

$$
\left(\begin{array}{l}
\widehat{E}(\varphi) \\
\widehat{\Omega}(\varphi)
\end{array}\right)=\frac{1}{2} \sum_{\boldsymbol{k}} w_{\boldsymbol{k}}\left(\begin{array}{c}
1 \\
k^{2}
\end{array}\right) \widehat{E}_{\boldsymbol{k}} .
$$

With this definition, the effective number of modes is

$$
\mathcal{M}=\sum_{k} w_{\boldsymbol{k}}
$$

For modes interior to the boundaries of the truncated $\boldsymbol{k}$ space, we consider only $w_{\boldsymbol{k}}=1$; however, the weightings of the edge or corner points may differ from 1 , as we will discuss later.

The thermal-equilibrium wave-number spectrum can now be shown to be

$$
E_{\boldsymbol{k}} \doteq\left\langle\widehat{E}_{\boldsymbol{k}}\right\rangle=\frac{1}{\alpha+\beta k^{2}} .
$$

It is worth giving the derivation of this result in detail in order to discuss and justify an annoying factor of 2 . By definition,

$$
\begin{aligned}
& \left\langle\widehat{E}_{\boldsymbol{k}}\right\rangle=\int\left(\prod_{\nu=1}^{\mathcal{M}} d \varphi_{r}^{(\nu)} d \varphi_{i}^{(\nu)}\right)\left(1+k^{2}\right)\left|\varphi_{\boldsymbol{k}}\right|^{2} \\
& \times Z^{-1} \exp \left[-\sum_{\bar{k}}^{\prime} w_{\overline{\boldsymbol{k}}}^{\prime}\left(1+\bar{k}^{2}\right)\left(\alpha+\beta \bar{k}^{2}\right)\left|\varphi_{\overline{\boldsymbol{k}}}\right|^{2}\right]
\end{aligned}
$$

of course, $\left|\varphi_{\boldsymbol{k}}\right|^{2}=\varphi_{k, r}^{2}+\varphi_{k, i}^{2}$. We have observed that $\varphi_{\boldsymbol{k}}$ and $\varphi_{-\boldsymbol{k}}$ are not independent, since by reality of $\varphi(\boldsymbol{x})$ one has $\varphi_{-\boldsymbol{k}}=\varphi_{\boldsymbol{k}}^{*}$. Therefore, the factor of $\frac{1}{2}$ in the definition (7) of the invariants has been eliminated in the

\footnotetext{
${ }^{3}$ For some related discussion of the short-wavelength limit, see M. Ottaviani and J. A. Krommes, Phys. Rev. Lett. 69, 2923 (1992).
}

exponent of Eq. (12) by replacing the unrestricted wavenumber sum in Eq. (7) by the appropriate $\sum_{k}^{\prime}$ over the half space of independent modes. (We allow for the possibility that the appropriate weight function $w_{\boldsymbol{k}}^{\prime}$ for $\sum_{\boldsymbol{k}}^{\prime}$ may differ from $w_{\boldsymbol{k}}$ on the boundary of the $\boldsymbol{k}$ space.) The result (11) then follows readily, at least for the interior modes, by performing a simple Gaussian integral. (Note that the partition function $Z$ factors.)

The discrete nature of the Fourier transform introduces subtlety into the evaluation of the invariants. It is numerically convenient to work with a square truncation, i.e., to sum over all retained modes, as this eliminates a time-consuming test to determine whether a mode should be retained. However, because of the symmetry properties $(3)$ the special modes $\left(n_{x}, n_{y}\right) \in$ $\left\{(0,0),\left(\frac{1}{2} M_{x}, 0\right),\left(0, \frac{1}{2} M_{y}\right),\left(\frac{1}{2} M_{x}, \frac{1}{2} M_{y}\right)\right\}$ are real. Since we are interested in fluctuations, we exclude the $(0,0)$ mode; however, the other modes must be counted appropriately. Now since the imaginary parts of those modes vanish identically, those parts are not available as independent coordinates for the canonical distribution. That thus has the schematic form (with $x$ and $y$ referring to the real and imaginary parts of any $E_{\boldsymbol{k}}$ )

$$
\mathcal{P}^{(\mathcal{M})} \sim \exp \left[-\left(\frac{x_{1}^{2}+y_{1}^{2}}{2 \sigma_{1}^{2}}\right)-\left(\frac{x_{2}^{2}}{2 \sigma_{2}^{2}}\right)\right],
$$

where the subscript 1 refers to an interior mode, 2 refers to a boundary mode, $x_{1}^{2}+y_{1}^{2}=\widehat{E}_{\boldsymbol{k}_{1}}, \sigma_{1}^{2} \doteq\left[2\left(\alpha+\beta k_{1}^{2}\right)\right]^{-1}$, and $\sigma_{2}$ must be determined such that the spectrum $E_{\boldsymbol{k}}$ is a smooth function of $\boldsymbol{k}$. Since $E_{\boldsymbol{k}_{1}}=\left\langle\left(x_{1}^{2}+y_{1}^{2}\right)\right\rangle=2 \sigma_{1}^{2}$ and $E_{\boldsymbol{k}_{2}}=\left\langle x_{2}^{2}\right\rangle=\sigma_{2}^{2}$, one deduces that the real modes must be weighted by a factor of $\frac{1}{2}$ in the summation $\sum^{\prime}$ that defines the invariants of the finite, discrete system. Thus in Fig. 1 the independent modes are indicated by squares and triangles; the triangles must be weighted by $\frac{1}{2}$. The corresponding weight function $w_{\boldsymbol{k}}^{\prime}$ is thus asymmetric when considered over the entire square; however, the reflection symmetries on the lattice guarantee that the four corner points are identical and that edge points are identical to appropriate points on the facing edge. Theoretically, then, one can replace $\sum_{k}^{\prime} w_{k}^{\prime}$ by $\frac{1}{2} \sum_{\boldsymbol{k}} w_{\boldsymbol{k}}$, where $w_{\boldsymbol{k}}$ corresponds to a weighting of the interior modes by 1 , the corner points by $\frac{1}{4}$, and the other edge points by $\frac{1}{2}$ (see Fig. 2). We will call this the weighted truncation. Note that numerically it is more efficient to employ the asymmetric weights $w_{\boldsymbol{k}}^{\prime}$; however, the $w_{\boldsymbol{k}}$ 's are easier to work with theoretically.

The spectrum (11) is identical to that for the 2D neutral fluid, which has been discussed in depth by Kraichnan [24]. For our later work in Sec. III, it is necessary to record here ${ }^{4}$ the portion of Kraichnan's analysis concerning the allowable values of $\alpha$ and $\beta$ and the relation

\footnotetext{
${ }^{4}$ A more concise version of this discussion can be found in Sec. 3.7.2 of Ref. [8].
} 


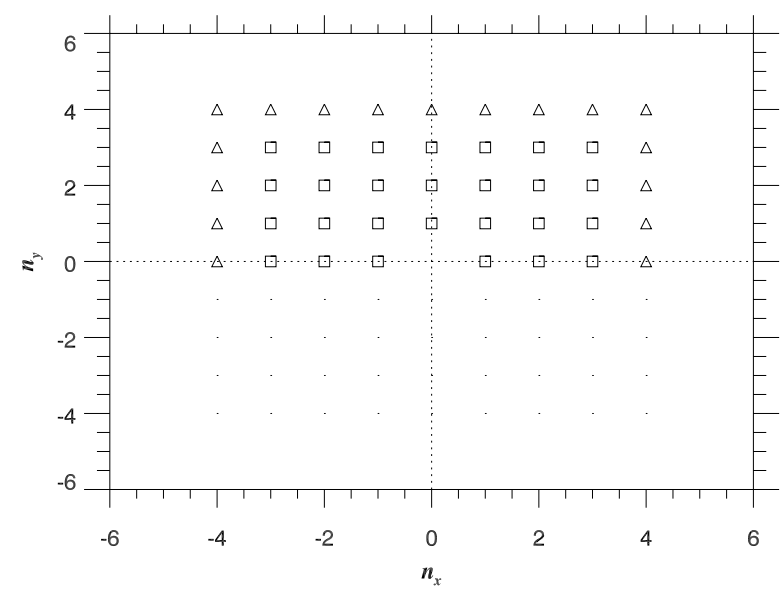

FIG. 1: Representative mode space for $M_{x}=3$. Independent modes are indicated by the open $n$-gons. The squares are weighted by 1 , the triangles are weighted by $\frac{1}{2}$.

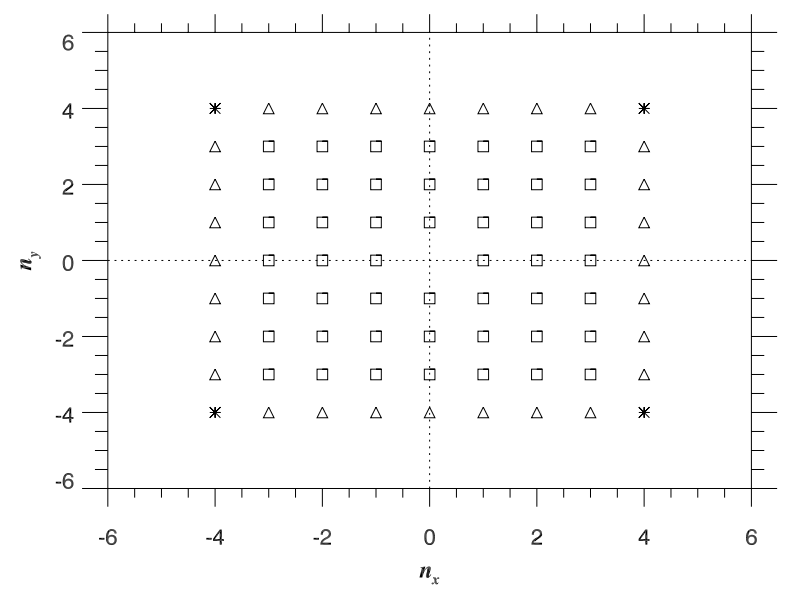

FIG. 2: The same mode space as in Fig. 1, but with symmetrized weightings. The corner points (asterisks) are weighted by $\frac{1}{4}$.

between $\{E, \Omega\}$ and $\{\alpha, \beta\}$ in a notation that emphasizes the discrete nature of the spectrum and allows for arbitrary weight functions $w_{\boldsymbol{k}}$. It is useful to couch the relations in terms of the energy and enstrophy per mode,

$$
\bar{E} \doteq E / \mathcal{M}, \quad \bar{\Omega} \doteq \Omega / \mathcal{M}
$$

It is also useful to define the dimensionless parameters

$$
\bar{\alpha} \doteq \alpha \bar{E}, \quad \bar{\beta} \doteq \beta \bar{E},
$$

and the ratio

$$
\widehat{\alpha} \doteq \alpha / \beta=\bar{\alpha} / \bar{\beta} .
$$

(This new use of the hat should cause no confusion in context.) One can then write the definitions (7) in the form

$$
\left(\frac{\bar{E}}{\Omega}\right)=\frac{1}{2 \beta}\left\langle\left(\begin{array}{c}
1 \\
k^{2}
\end{array}\right)\left(\frac{1}{\widehat{\alpha}+k^{2}}\right)\right\rangle_{k},
$$

where the notation $\langle\ldots\rangle_{\boldsymbol{k}}$ denotes the average over the discrete, truncated wave-number spectrum:

$$
\langle A\rangle_{\boldsymbol{k}} \doteq \frac{1}{\mathcal{M}} \sum_{\boldsymbol{k}} w_{\boldsymbol{k}} A_{\boldsymbol{k}} .
$$

It is useful to define the ratio of enstrophy to energy, a dimensionless quantity that should be thought of as the square of a (dimensionless) wave number $\kappa$ :

$$
\kappa^{2} \doteq \Omega / E=\bar{\Omega} / \bar{E} .
$$

Then, upon adding and subtracting $\widehat{\alpha}$ to the numerator of the expression (17) for $\bar{\Omega}$, one finds the convenient expression

$$
\kappa^{2}=(2 \bar{\beta})^{-1}-\widehat{\alpha},
$$

where

$$
\bar{\beta}=\frac{1}{2}\left\langle\frac{1}{\widehat{\alpha}+k^{2}}\right\rangle_{k} .
$$

Equation (20) can be used to prove an important constraint that will be very useful in the subsequent Monte Carlo calculations. Define

$$
\bar{U} \doteq 2(\alpha \bar{E}+\beta \bar{\Omega})
$$

[twice the average of the exponent of the Gibbs distribution $\mathcal{P}(\varphi) \propto \exp (-\alpha \widehat{E}-\beta \widehat{\Omega})]$. One has

$$
\begin{aligned}
\bar{U} & =2 \beta \bar{E}(\alpha / \beta+\bar{\Omega} / \bar{E}) \\
& =2 \bar{\beta}\left(\widehat{\alpha}+\kappa^{2}\right) .
\end{aligned}
$$

Upon using Eq. (20), one finally finds

$$
\bar{U}=1 .
$$

This is a simple generalization of the result that for a $1 \mathrm{D}$ PDF of the form $P(x) \propto \exp \left(-\alpha x^{2}\right)$, one has $2 \alpha\left\langle x^{2}\right\rangle=1$. Its importance is that for a general non-Gaussian distribution one will have $\bar{U} \neq 1$, so the approach of $\bar{U}$ to 1 can be used as a convenient (and in practice very sensitive) diagnostic of the convergence of the Monte Carlo Markov chain to the desired asymptotic equilibrium distribution. (See Sec. III B.)

The parameter space can now be analyzed by demanding that $\bar{E}, \bar{\Omega}$, and $E_{k}$ be non-negative. Considered as a function of $\widehat{\alpha}, E_{k}$ is singular at $\widehat{\alpha}=-k_{\min }^{2}$ and $\widehat{\alpha}=-k_{\max }^{2}$, and one can determine that the region $-k_{\max }^{2}<\widehat{\alpha}<-k_{\min }^{2}$ is forbidden since one or more of the $E_{\boldsymbol{k}}$ would be negative. To analyze the behavior in the vicinity of $\widehat{\alpha}=-k_{\min }^{2}$, we write $\widehat{\alpha}=-k_{\min }^{2}+\epsilon / \mathcal{M}$. Then $\bar{\beta} \sim \epsilon^{-1} \rightarrow+\infty$ as $\epsilon \rightarrow 0_{+}$. For fixed $\bar{E}$, which we always assume in considering the various limiting cases, one sees that also $\beta \rightarrow+\infty$. The behavior of $\bar{\alpha} \doteq \alpha \bar{E}$ follows from $\bar{\alpha}=\widehat{\alpha} \bar{\beta}=\left(-k_{\min }^{2}+\epsilon / \mathcal{M}\right) \bar{\beta} \approx-k_{\min }^{2} \bar{\beta} \rightarrow-\infty$. Also, $\kappa^{2} \rightarrow-\widehat{\alpha} \rightarrow k_{\min }^{2}$. Symmetrical behavior ensues in the vicinity of $\widehat{\alpha}=-k_{\max }^{2}-\epsilon / \mathcal{M}$, with the roles of $\alpha$ 
and $\beta, k_{\min }$ and $k_{\max }$ reversed. The other interesting points are $\widehat{\alpha}=0$ and $\widehat{\alpha}= \pm \infty$. Define the special wave numbers $k_{a}$ and $k_{b}$ according to

$$
\begin{aligned}
k_{a}^{2} & \doteq\left\langle k^{-2}\right\rangle_{\boldsymbol{k}}^{-1}, \\
k_{b}^{2} & =\left\langle k^{2}\right\rangle_{\boldsymbol{k}} .
\end{aligned}
$$

[That $k_{b} \geq k_{a}$ is a consequence of a Schwartz inequality applied to the identity $\left\langle\left(k^{2}\right)\left(k^{-2}\right)\right\rangle=1$.] Then at $\widehat{\alpha}=0$ one finds $\bar{E}=\left(2 \beta k_{a}^{2}\right)^{-1}, \bar{\Omega}=(2 \beta)^{-1}$, and $\kappa^{2}=k_{a}^{2}$. As $\widehat{\alpha} \rightarrow+\infty$ one obtains $\bar{E}=(2 \alpha)^{-1}, \bar{\Omega}=(2 \alpha)^{-1} k_{b}^{2}$, and $\kappa^{2}=k_{b}^{2}$. Since for fixed $\bar{E} \alpha$ remains finite, we see that $\beta \rightarrow 0_{+}$as $\widehat{\alpha} \rightarrow+\infty$. The point $\widehat{\alpha}=-\infty$ is obtained continuously from $\widehat{\alpha}=+\infty$ as $\beta$ passes continuously through 0 from above. One thus identifies three regimes:

$$
\begin{aligned}
\text { Regime I: } k_{\min }^{2} & \leq \kappa^{2} \leq k_{a}^{2}, \\
-k_{\min }^{2} & \leq \widehat{\alpha} \leq 0, \\
-\infty & \leq \bar{\alpha} \leq 0, \\
\infty & \geq \bar{\beta} \geq k_{a}^{-1} ; \\
\text { Regime II: } \quad k_{a}^{2} & \leq \kappa^{2} \leq k_{b}^{2}, \\
0 & \leq \widehat{\alpha}<\infty, \\
0 & \leq \bar{\alpha} \leq 1 / 2, \\
k_{a}^{-1} & \geq \bar{\beta} \geq 0 ; \\
k_{b}^{2} & \leq \kappa^{2} \leq k_{\max }^{2}, \\
-\infty & <\widehat{\alpha} \leq-k_{\max }^{2}, \\
1 / 2 & \leq \bar{\alpha} \leq \infty, \\
0 & \geq \bar{\beta} \geq-\infty .
\end{aligned}
$$

The qualitative features of this behavior are summarized in Figs. 3 and 4. Those figures should not be used for precise quantitative work, since they actually plot the approximation obtained by assuming that the spectrum is dense and spherically truncated [24],

$$
\begin{gathered}
\mathcal{M} \approx \pi\left(k_{\max }^{2}-k_{\min }^{2}\right), \\
\bar{\beta} \approx \frac{1}{2} \ln \left(\frac{\widehat{\alpha}+k_{\max }^{2}}{\bar{\alpha}+k_{\min }^{2}}\right) /\left(k_{\max }^{2}-k_{\min }^{2}\right), \\
k_{a}^{2} \approx \frac{k_{\max }^{2}-k_{\min }^{2}}{\ln \left(k_{\max }^{2} / k_{\min }^{2}\right)}, \\
k_{b}^{2} \approx \frac{1}{2}\left(k_{\min }^{2}+k_{\max }^{2}\right) .
\end{gathered}
$$

Regime II is the most intuitively familiar regime of positive temperatures, bounded on the left by the enstrophyequipartition state $\widehat{\alpha}=0, \Omega_{k}=\bar{\Omega}=(2 \beta)^{-1}=k_{a}^{2} \bar{E}$ and on the right by the energy-equipartition state $\beta=0$, $E_{k}=\bar{E}=(2 \alpha)^{-1}=\bar{\Omega} / k_{b}^{2}$. Regime I corresponds to negative- $\alpha$ states; symmetrically, regime III corresponds

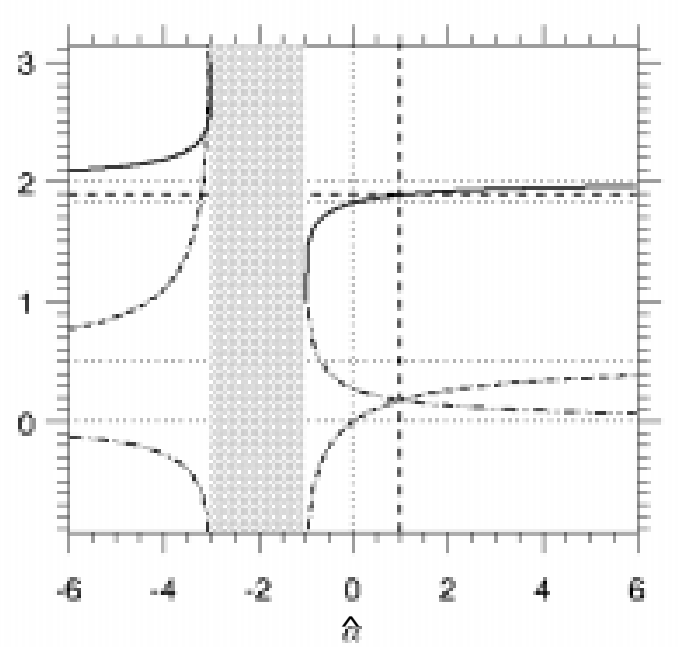

FIG. 3: Important quantities for two-temperature equilibria, plotted vs $\widehat{\alpha} \doteq \alpha / \beta$. The gray region is the forbidden zone $-k_{\max }^{2}<\widehat{\alpha}<-k_{\min }^{2}$. Solid line, $\kappa^{2}(\widehat{\alpha}) ;$ dash-dotted line, $\bar{\alpha}(\widehat{\alpha})$; triple dash-dotted line, $\bar{\beta}(\widehat{\alpha})$. The dashed lines indicate the special noise case $\widehat{\alpha}=1, \kappa^{2}=\kappa_{1}^{2}$. The horizontal dotted lines indicate, from bottom to top, the special cases $\kappa^{2}=$ $\left(0, \frac{1}{2}, k_{a}^{2}, k_{b}^{2}\right)$.

to negative- $\beta$ states. States with highly negative $\alpha$ have the longest wavelength modes excited to very high levels. For states with highly negative $\beta$, the excitation is concentrated at the shortest wavelength modes.

The precise numerical values of the characteristic wave numbers $k_{a}$ and $k_{b}$ are not accessible from the continuum approximations (26); they must be determined numerically as a function of the number of discrete modes. We generally consider $M_{x}=M_{y}=2^{m}$. In Tables 1-3 we tabulate the values of $k_{a}, k_{b}$, and the additional important wave number $\kappa_{1}$ [defined later; see Eq. (39b)] normalized to $k_{\min }$ for representative $m$ 's and the truncations defined by

$$
\begin{aligned}
& \text { Spherical: } w_{\boldsymbol{k}}= \begin{cases}1 & 1 \leq k \leq M_{x} / 2 \\
0 & \text { otherwise, }\end{cases} \\
& \text { Square: } w_{\boldsymbol{k}}= \begin{cases}1 & \begin{array}{l}
-M_{x} / 2 \leq k_{x} \leq M_{x} / 2 \\
-M_{y} / 2 \leq k_{y} \leq M_{y} / 2 \\
\boldsymbol{k} \neq 0
\end{array} \\
0 & \text { otherwise, }\end{cases} \\
& \text { Weighted: } w_{\boldsymbol{k}}= \begin{cases}d_{\boldsymbol{k}} & \begin{array}{l}
-M_{x} / 2 \leq k_{x} \leq M_{x} / 2 \\
-M_{y} / 2 \leq k_{y} \leq M_{y} / 2 \\
\boldsymbol{k} \neq 0
\end{array} \\
0 & \text { otherwise, }\end{cases}
\end{aligned}
$$

where

$$
d_{k} \doteq \begin{cases}1 & \text { (interior point) } \\ 1 / 2 & \text { (edge but not corner) } \\ 1 / 4 & \text { (corner) }\end{cases}
$$




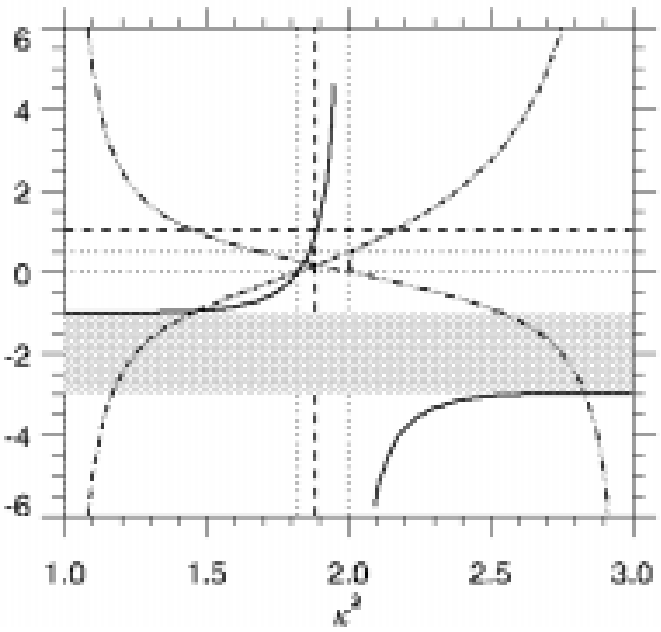

FIG. 4: Important quantities for two-temperature equilibria, plotted vs $\kappa^{2} \doteq \Omega / E$. Gray region: forbidden zone $-k_{\max }^{2}<\widehat{\alpha}<-k_{\min }^{2}$. Solid line, $\widehat{\alpha}\left(\kappa^{2}\right)$; dash-dotted line, $\bar{\alpha}\left(\kappa^{2}\right)$; triple dash-dotted line, $\bar{\beta}\left(\kappa^{2}\right)$. The dashed lines indicated the special noise case $\widehat{\alpha}=1, \kappa^{2}=\kappa_{1}^{2}$. The vertical dotted lines indicate, from left to right, $k_{a}^{2}$ and $k_{b}^{2}$. The horizontal dotted lines indicate, from bottom to top, the special cases $\bar{\beta}\left(k_{b}^{2}\right)=0$ and $\bar{\alpha}\left(k_{a}^{2}\right)=\frac{1}{2}$.

For the spherical truncation one has $k_{\max } / k_{\min }=\frac{1}{2} M_{x}=$ $2^{m-1}$; for the other truncations $k_{\max }$ is $\sqrt{2}$ times larger.

For given $\bar{E}$ and $\bar{\Omega}$, the associated $\alpha$ and $\beta$ are determined as follows. We replace the set $\{\bar{E}, \bar{\Omega}\}$ by $\left\{\bar{E}, \kappa^{2}\right\}$. The relation (20),

$$
\kappa^{2}=\kappa^{2}(\widehat{\alpha})=[2 \bar{\beta}(\widehat{\alpha})]^{-1}-\widehat{\alpha},
$$

can be inverted (numerically, in practice) to give $\widehat{\alpha}\left(\kappa^{2}\right)$. The function $\bar{\beta}\left(\kappa^{2}\right)$ [Eq. (21)] is then known from Eq. (20) as

$$
\bar{\beta}\left(\kappa^{2}\right)=\frac{1}{2}\left[\widehat{\alpha}\left(\kappa^{2}\right)+\kappa^{2}\right]^{-1} .
$$

The function $\bar{\alpha}\left(\kappa^{2}\right) \doteq \alpha \bar{E}$ follows from

$$
\bar{\alpha}\left(\kappa^{2}\right)=\widehat{\alpha}\left(\kappa^{2}\right) \bar{\beta}\left(\kappa^{2}\right) .
$$

Finally, the absolute inverse temperatures follow from Eqs. (15).

Instead of specifying $\bar{E}$ and $\bar{\Omega}$, it is often desirable in the applications to specifically select a particular temperature regime, e.g., a state with highly negative $\alpha$. Then one first specifies $\kappa$ instead of determining it by computing the ratio of known values of $\bar{E}$ and $\bar{\Omega}$; one then proceeds as before to determine $\bar{\alpha}$ and $\bar{\beta}$. The ratio $\bar{\Omega} / \bar{E}=\Omega / E$ is immediately given by $\kappa^{2}$; if one requires absolute values of $E, \Omega, \alpha$, or $\beta$, either $\bar{E}$ or $\bar{\Omega}$ must be additionally specified.

\section{Equal-temperature equilibrium}

One situation in which $\bar{E}$ and $\bar{\Omega}$ are known arises when one considers the noise in the common initialization in which a finite number $N$ of particles are distributed uniformly and independently in the box. The particle density as a function of continuous position $\boldsymbol{x}$ is $n(\boldsymbol{x})=$ $\sum_{\ell} \delta\left(\boldsymbol{x}-\boldsymbol{x}^{(\ell)}\right)$, where on the lattice one must interpret $\delta\left(\boldsymbol{x}_{\boldsymbol{j}}\right)$ as $\boldsymbol{\Delta}\left(\boldsymbol{x}_{\boldsymbol{j}}\right), \boldsymbol{\Delta}(\boldsymbol{x})$ being the periodic delta function $\boldsymbol{\Delta}\left(\boldsymbol{x}_{\boldsymbol{j}}\right) \doteq V^{-1} \sum_{\boldsymbol{k}} e^{i \boldsymbol{k} \cdot \boldsymbol{x}_{\boldsymbol{j}}}$ obeying $\boldsymbol{\Delta}(0)=\Delta V^{-1}$. The mean particle density is $\bar{n}=V^{-1} \int d \boldsymbol{x} n(\boldsymbol{x})=N / V$, and the power spectrum of the density fluctuation $\delta n_{\boldsymbol{k}} \doteq$ $n_{k}-\bar{n}$ is readily determined to be

$$
\left\langle\left|\delta n_{\boldsymbol{k}} / \bar{n}\right|^{2}\right\rangle= \begin{cases}N^{-1} & (\boldsymbol{k} \neq 0) \\ 0 & (\boldsymbol{k}=0) .\end{cases}
$$

In subsequent formulas it will be understood that we consider only $\boldsymbol{k} \neq 0$.

In Hasegawa-Mima dynamics the above density can be identified with the density of ion gyrocenters. From the gyrokinetic Poisson equation for adiabatic electrons (Appendix A),

$$
\left(1-\nabla^{2}\right) \varphi=n(\boldsymbol{x}) / \bar{n},
$$

one finds $\left\langle\left|\delta \varphi_{\boldsymbol{k}}\right|^{2}\right\rangle=\left(1+k^{2}\right)^{-2} N^{-1}$, so the quantity $E_{\boldsymbol{k}} \doteq$ $\left(1+k^{2}\right)\left\langle\left|\delta \varphi_{\boldsymbol{k}}\right|^{2}\right\rangle[$ see Eq. (8)] is

$$
E_{k}=\left(\frac{1}{1+k^{2}}\right) \frac{1}{N}
$$

Upon comparing the result (33) with Eq. (11), one sees that the spectrum of uniform random particle noise has energy and enstrophy compatible with a canonical (and Gaussian) equilibrium potential distribution with equal and positive inverse temperatures

$$
\alpha_{1}=\beta_{1}=N .
$$

(Here the 1 subscript reminds one that for this state $\widehat{\alpha}=\alpha_{1} / \beta_{1}=1$.) However, it is very important to realize that the full PDF of this noise is noncanonical and non-Gaussian. That is, the probability density $\mathcal{P}_{0}(\varphi)$ of realizing a set of Fourier amplitudes $\{\varphi\}$ is not proportional to

$$
\mathcal{P}_{0}^{(\mathrm{G})} \propto \exp \left[-\sum_{\boldsymbol{k}} N\left(1+k^{2}\right) \widehat{E}_{\boldsymbol{k}}\right]
$$

even though the mean $E_{\boldsymbol{k}}$ (proportional to the second moment of the potential) is correctly predicted by the Gibbsian and Gaussian result. The true $\mathcal{P}_{0}$ is much more complicated because of higher-order correlations arising from the complicated nonlinear form of the Poisson equation considered as a function of the particle positions, and this observation has important consequences for our subsequent application of the Monte Carlo method. The correct $\mathcal{P}_{0}(\varphi)$ is computed in detail in Appendixes B and $\mathrm{C}$. Here we merely point out that the difference 


\begin{tabular}{|c|c|c|c|c|c|c|c|c|c|c|c|}
\hline \multirow{2}{*}{$\frac{m}{2}$} & \multicolumn{2}{|c|}{ \# of modes } & \multicolumn{2}{|c|}{$k_{a}$} & \multicolumn{2}{|c|}{$k_{1}$} & \multicolumn{2}{|r|}{$\bar{\alpha}$} & \multicolumn{2}{|c|}{$k_{b}$} & \multirow{2}{*}{$\frac{k_{\max }}{2}$} \\
\hline & 12 & $(-0.2146)$ & 1.309 & $(0.12355)$ & 1.517 & $(0.038896)$ & 0.477 & $(-0.0036536)$ & 1.528 & $(0.035098)$ & \\
\hline 3 & 48 & $(-0.018252)$ & 2.069 & $(0.12404)$ & 2.764 & $(0.033887)$ & 0.431 & $(-0.0094577)$ & 2.828 & $(0.030776)$ & 4 \\
\hline 4 & 196 & $(0.0097976)$ & 3.546 & $(0.097497)$ & 5.219 & (0.017103) & 0.318 & $(-0.012419)$ & 5.617 & $(0.014927)$ & 8 \\
\hline 5 & 796 & $(0.0064147)$ & 6.312 & $(0.074295)$ & 9.496 & $(0.0075945)$ & 0.172 & $(-0.0098883)$ & 11.270 & $(0.0058683)$ & 16 \\
\hline 6 & 3208 & $(0.0018233)$ & 11.477 & $(0.058483)$ & 16.768 & $(0.0032807)$ & 0.072 & $(-0.0055911)$ & 22.603 & $(0.0015546)$ & 32 \\
\hline 7 & 12852 & $(0.00099766)$ & 21.157 & $(0.048752)$ & 29.489 & $(0.0020646)$ & 0.026 & $(-0.003904)$ & 45.230 & $(0.00066221)$ & 64 \\
\hline 8 & 51432 & $(0.00071381)$ & 39.437 & $(0.041868)$ & 52.501 & $(0.001531)$ & 0.008 & $(-0.0030035)$ & 90.476 & $(0.00039774)$ & 128 \\
\hline
\end{tabular}

TABLE I: Important parameters for a discrete spectrum in the spherical truncation. The parenthesized numbers are the relative differences between the discrete results and the continuum approximation described by Eqs. (26). All $k$ 's are normalized to $k_{\min }$.

\begin{tabular}{|c|c|c|c|c|c|c|c|c|c|c|c|}
\hline \multirow{2}{*}{$\frac{m}{2}$} & \multicolumn{2}{|c|}{ \# of modes } & \multicolumn{2}{|c|}{$k_{a}$} & \multicolumn{2}{|c|}{$k_{1}$} & \multicolumn{2}{|r|}{$\bar{\alpha}$} & \multicolumn{2}{|c|}{$k_{b}$} & \multirow{2}{*}{$\frac{k_{\max }}{2.828}$} \\
\hline & 24 & $(-0.5)$ & 1.624 & $(-0.094167)$ & 2.028 & $(-0.22162)$ & 0.478 & $(0.017304)$ & 2.041 & $(-0.2254)$ & \\
\hline 3 & 80 & $(-0.25)$ & 2.505 & $(-0.071519)$ & 3.588 & $(-0.19649)$ & 0.438 & $(0.045849)$ & 3.674 & $(-0.20651)$ & 5.657 \\
\hline 4 & 288 & $(-0.125)$ & 4.149 & $(-0.061972)$ & 6.472 & $(-0.15684)$ & 0.343 & $(0.1001)$ & 6.940 & $(-0.17857)$ & 11.314 \\
\hline 5 & 1088 & $(-0.0625)$ & 7.216 & $(-0.060305)$ & 11.487 & $(-0.1246)$ & 0.204 & $(0.16034)$ & 13.472 & $(-0.15858)$ & 22.627 \\
\hline 6 & 4224 & $(-0.03125)$ & 12.960 & $(-0.062585)$ & 20.013 & $(-0.10732)$ & 0.093 & $(0.19825)$ & 26.536 & $(-0.14688)$ & 45.255 \\
\hline 7 & 16640 & $(-0.015625)$ & 23.768 & $(-0.066453)$ & 34.858 & $(-0.10105)$ & 0.035 & $(0.21727)$ & 52.664 & $(-0.14058)$ & 90.510 \\
\hline 8 & 66048 & $(-0.0078125)$ & 44.214 & $(-0.07068)$ & 61.545 & $(-0.1)$ & 0.012 & $(0.22781)$ & 104.920 & $(-0.13732)$ & 181.019 \\
\hline
\end{tabular}

TABLE II: Important parameters for a discrete spectrum in the square truncation.

between $\mathcal{P}_{0}$ and a Gaussian distribution is intimately related to the difference between the random-phase approximation and the assumption of Gaussian statistics. That is, the characteristic function (moment generating function, or Fourier transform of the $\mathrm{PDF}^{5}$ ) for the random variable $\psi \doteq \sin \theta$, where $\theta$ is distributed uniformly on the interval $[0,2 \pi)$, is $P_{k}=J_{0}(k)$, where $J_{0}$ is the ordinary Bessel function of the first kind. Such a variable has variance $\left\langle\delta \psi^{2}\right\rangle=\frac{1}{2}$. The characteristic function for a Gaussian with variance $\frac{1}{2}$ is $\exp \left(-\frac{1}{4} k^{2}\right)$. Both this function and $J_{0}(k)$ behave as $1-\frac{1}{4} k^{2}+O\left(k^{4}\right)$ for small $k$, but differ in the terms of $O\left(k^{4}\right)$ and higher. Put another way, the logarithm of $P_{k}$ is the cumulant generating function [25]:

$$
\ln P_{k}=\sum_{l=1}^{\infty} \frac{(-i k)^{l}}{l !} C_{l}
$$

One readily obtains, for example, $C_{2}=\frac{1}{2}$ and $C_{4}=-\frac{3}{8}$; in general, the random-phase approximation has nonvanishing cumulants of all even orders. If $\psi$ were Gaussian, on the other hand, all cumulants higher than the second would vanish. Unfortunately, "random phase" is often used synonymously with "Gaussian" in many plasmaphysics discussions. Although in some cases this does not matter, in general it is quite misleading. In the present application, the difference is crucial.

\footnotetext{
5 An introduction (with references) to statistical methods can be
} found in Ref. [8].
The precise energy per mode,

$$
\bar{E}=\frac{1}{N}\left\langle\frac{1}{1+k^{2}}\right\rangle_{\boldsymbol{k}},
$$

must be computed numerically as a function of the wavenumber cutoffs, but it is important to note that the normalized quantities

$$
\bar{\alpha}_{1}=\bar{\beta}_{1}=\left\langle\frac{1}{1+k^{2}}\right\rangle_{k}
$$

are of order unity (independent of $N$ ). The associated $\kappa$ corresponds to that point $\kappa_{1}$ in Fig. 3 where the curves $\bar{\alpha}\left(\kappa^{2}\right)$ and $\bar{\beta}\left(\kappa^{2}\right)$ intersect, namely,

$$
\begin{gathered}
\widehat{\alpha}=1 \\
\kappa_{1}^{2}=\frac{1}{2}\left\langle\frac{1}{1+k^{2}}\right\rangle_{k}^{-1}-1 .
\end{gathered}
$$

The values of $\kappa_{1}$ and the associated $\bar{\alpha}_{1}=\bar{\beta}_{1}=[2(1+$ $\left.\left.\kappa_{1}^{2}\right)\right]^{-1}$ are tabulated in Tables I-III as functions of $m$.

\section{DETERMINING AND SAMPLING FROM THE PARTICLE PROBABILITY DISTRIBUTION}

By definition, generating a particle state means sampling from the $N$-particle PDF $P^{(N)}(\Gamma)$. Two questions arise: (1) What is $P^{(N)}(\Gamma)$ ? (2) Given $P^{(N)}(\Gamma)$, how does one construct an appropriate sampling algorithm? As a trivial example, suppose that the particles are to be distributed independently and uniformly in a 


\begin{tabular}{|c|c|c|c|c|c|c|c|c|c|c|c|}
\hline \multirow{2}{*}{$\frac{m}{2}$} & \multicolumn{2}{|c|}{ \# of modes } & \multicolumn{2}{|c|}{$k_{a}$} & \multicolumn{2}{|c|}{$k_{1}$} & \multicolumn{2}{|r|}{$\bar{\alpha}$} & \multicolumn{2}{|c|}{$k_{b}$} & \multirow{2}{*}{$\frac{k_{\max }}{2.828}$} \\
\hline & 15 & $(-0.2)$ & 1.421 & $(0.034988)$ & 1.777 & $(-0.11148)$ & 0.483 & $(0.0070936)$ & 1.789 & $(-0.11612)$ & \\
\hline 3 & 63 & $(-0.047619)$ & 2.298 & $(0.012321)$ & 3.270 & $(-0.11822)$ & 0.448 & $(0.0239)$ & 3.343 & $(-0.12785)$ & 5.657 \\
\hline 4 & 255 & $(-0.011765)$ & 3.952 & $(-0.015118)$ & 6.151 & $(-0.1128)$ & 0.353 & $(0.066577)$ & 6.570 & $(-0.13232)$ & 11.314 \\
\hline 5 & 1023 & $(-0.0029326)$ & 7.031 & $(-0.035521)$ & 11.201 & $(-0.10224)$ & 0.210 & $(0.12659)$ & 13.083 & $(-0.13355)$ & 22.627 \\
\hline 6 & 4095 & $(-0.0007326)$ & 12.785 & $(-0.049804)$ & 19.765 & $(-0.096126)$ & 0.095 & $(0.17423)$ & 26.137 & $(-0.13387)$ & 45.255 \\
\hline 7 & 16383 & $(-0.00018312)$ & 23.603 & $(-0.059945)$ & 34.638 & $(-0.095342)$ & 0.035 & $(0.20303)$ & 52.261 & $(-0.13395)$ & 90.510 \\
\hline 8 & 65535 & $(-4.5777 \mathrm{e}-05)$ & 44.057 & $(-0.06739)$ & 61.344 & $(-0.097068)$ & 0.012 & $(0.22002)$ & 104.514 & $(-0.13397)$ & 181.019 \\
\hline
\end{tabular}

TABLE III: Important parameters for a discrete spectrum in the weighted truncation.

box of volume $V=L^{d}$. Independence means $P^{(N)}(\Gamma)=$ $\prod_{\ell} P^{(1)}\left(\boldsymbol{x}^{(\ell)}\right)$, and homogeneity implies $P^{(1)}(\boldsymbol{x})=V^{-1}$, so $P^{(N)}(\Gamma)=V^{-N}$. A straightforward sampling algorithm consists of initializing each Cartesian component of the $\boldsymbol{x}^{(\ell)}$ with random numbers drawn from a distribution uniform on $[0, L)$.

Unfortunately, as we have remarked, the present problem differs from the conventional one of equilibrium statistical mechanics in that we are given $\mathcal{P}^{(\mathcal{M})}(\varphi)$ (the PDF of the Fourier components) rather than $P^{(N)}(\Gamma)$ (the PDF of the particles). The $\varphi_{\boldsymbol{k}}$ 's and $\boldsymbol{x}^{(\ell)}$ 's are related via the Fourier transform of the gyrokinetic Poisson equation (32),

$$
\left(1+k_{\nu}^{2}\right) \varphi_{\boldsymbol{k}_{\nu}}=\frac{1}{N} \sum_{\ell=1}^{N} e^{-i \boldsymbol{k}_{\nu} \cdot \boldsymbol{x}^{(\ell)}} \quad(\nu=1, \ldots, \mathcal{M}),
$$

where the factor of $N^{-1}$ is equal to $(\bar{n} V)^{-1}$, the $V$ arising from the Fourier transform convention (1a). This complicated nonlinear relation is a system of $2 \mathcal{M}$ real equations involving $d N$ Cartesian positions, where $d=2$ is the number of spatial dimensions. For $2 \mathcal{M}=d N$ or $\mathcal{M}=N$ one expects that it should be possible in general to invert this relation and thus determine $P^{(N)}(\Gamma)$ in terms of the Jacobian of the transformation (40). However, it is very unusual that $\mathcal{M}=N$; usually the desire for low sampling noise dictates $N \gg \mathcal{M}$, so the system is underdetermined; many particle (micro-)states are compatible with a given set of Fourier amplitudes (macrostate). Furthermore, even if the inversion were possible, the resulting $P^{(N)}(\Gamma)$ would be extremely complicated, so a suitable sampling algorithm would probably not be apparent.

As we have suggested, it is possible to avoid these difficulties by employing a Monte Carlo technique. However, before turning to that we wish to discuss an alternative possible procedure that, although flawed, provides additional insight and motivation.

\section{A. An impractical but instructive procedure}

The idea is based on two observations: (i) usually $N \gg \mathcal{M}$; (ii) $\mathcal{P}^{(\mathcal{M})}(\varphi)$ is Gaussian. Consider dividing the particle population into $G$ independent groups of
$\mathcal{M}$ particles each, choosing $N$ such that $N=G \mathcal{M}$. Then consider the $G$ systems

$$
\begin{aligned}
\left(1+k_{\nu}^{2}\right) \psi_{\boldsymbol{k}_{\nu}}^{(g)}= & \frac{1}{N} \sum_{\ell \in g} e^{-i \boldsymbol{k}_{\nu} \cdot \boldsymbol{x}^{(\ell)}} \\
& (\nu=1, \ldots, \mathcal{M} ; g=1, \ldots, G),
\end{aligned}
$$

where $\sum_{\ell \in g}$ means sum over the $\mathcal{M}$ particles in group $g$. If one constructs

$$
\varphi_{\boldsymbol{k}}=\sum_{g=1}^{G} \psi_{\boldsymbol{k}}^{(g)}
$$

then $\varphi_{\boldsymbol{k}}$ obeys

$$
\begin{aligned}
\left(1+k^{2}\right) \varphi_{\boldsymbol{k}} & =\sum_{g=1}^{G}\left(\frac{1}{N} \sum_{\ell \in g} e^{-i \boldsymbol{k} \cdot \boldsymbol{x}^{(\ell)}}\right) \\
& =\frac{1}{N} \sum_{\ell} e^{-i \boldsymbol{k} \cdot \boldsymbol{x}^{(\ell)}} .
\end{aligned}
$$

Therefore, if the $\boldsymbol{x}^{(\ell)}$ exist the Poisson equation will be satisfied. Furthermore, the variance of $\varphi_{\boldsymbol{k}}$ is related to the variance $\sigma_{k}^{2} \doteq\left\langle\left|\psi_{\boldsymbol{k}}\right|^{2}\right\rangle$ by

$$
\begin{aligned}
\left\langle\left|\varphi_{\boldsymbol{k}}\right|^{2}\right\rangle & =\sum_{g, g^{\prime}}\left\langle\psi_{\boldsymbol{k}}^{(g)} \psi_{\boldsymbol{k}}^{\left(g^{\prime}\right) *}\right\rangle \\
& =\sum_{g}\left\langle\left|\psi_{\boldsymbol{k}}^{(g)}\right|^{2}\right\rangle \\
& =G \sigma_{\boldsymbol{k}}^{2},
\end{aligned}
$$

where we exploited the assumption that the systems are independent. Therefore the variance of $\psi_{\boldsymbol{k}}$ is related in a simple way to the desired variance of $\varphi_{\boldsymbol{k}}$.

The proposal is now to sample, for each $g$, a collection of $\mathcal{M} \psi_{\boldsymbol{k}}$ 's from an appropriate PDF (independent of $g$ ) whose variance is $\sigma_{\boldsymbol{k}}^{2}$. (Appropriate care must be taken to satisfy the reality conditions.) This is easy with the aid of standard numerical software packages. Then solve the nonlinear system to obtain $\mathcal{M} \boldsymbol{x}^{(\ell)}$ 's. Those $\boldsymbol{x}^{(\ell)}$ 's are guaranteed to be compatible with the given variance of the $\varphi_{\boldsymbol{k}}$.

If the PDF for the $\psi_{\boldsymbol{k}}^{(g)}$ were Gaussian, then since the sum of Gaussian random variables is again Gaussian the $\varphi_{\boldsymbol{k}}$ would be Gaussian and we would have constructed a 
valid particle realization. Unfortunately, Gaussian $\psi_{\boldsymbol{k}}^{(g)}$ are not permitted. The modulus of Eq. (41) obeys

$$
\left(1+k^{2}\right)\left|\psi_{\boldsymbol{k}}^{(g)}\right|=N^{-1}\left|\sum_{\ell \in g} \exp \left(-i \boldsymbol{k} \cdot \boldsymbol{x}^{(\ell)}\right)\right| \leq G / N .
$$

If $\psi_{\boldsymbol{k}}^{(g)}$ were sampled from a Gaussian distribution, there would be a finite probability of obtaining a $\psi$ such that $|\psi|>G / N$. For such $\psi$, the solution of Eq. (40) will not exist. One must therefore sample from a bounded PDF. If that obeys appropriate constraints, one can appeal to the central limit theorem to argue that $\varphi_{\boldsymbol{k}}$ is asymptotically Gaussian for $G \gg 1$. In practice, this may not be the regime of interest, since we often consider relatively small numbers of particles. Furthermore, practical difficulties are certain to ensue. For example, the usual methods for solving nonlinear systems such as Eq. (41) involve some sort of functional iteration. However, there are no guarantees that such iteration will converge unconditionally. Nonconvergence may occur because of a poor initial guess, the existence of multiple solutions, and/or degeneracies associated with the regular nature of the wave-number lattice. Although one might think of solutions for each of these difficulties, it is clear that the proposed method is cumbersome at best and difficult to fully automate. Moreover, it suffers from the conceptual disadvantage that the realizations it generates are statistically only approximately valid for fixed, finite $N$.

Fortunately, the deficiencies of the procedure suggest a more fruitful line of approach. What is needed is a way of selecting $N$-particle states that are as random as possible consistent with the desired Gaussian statistics on the Fourier amplitudes while guaranteeing unconditional convergence to an acceptable sequence of states. These criteria are satisfied by the Monte Carlo algorithm of $\mathrm{M}(\mathrm{RT})^{2}$.

\section{B. The $\mathrm{M}(\mathrm{RT})^{2}$ algorithm}

Monte Carlo procedures [20, 26, 27] are often introduced from the point of view of the evaluation of multidimensional integrals. Although we will not need to evaluate such integrals explicitly, the application of computing integrals does provide fundamental motivation. Thus an integral $I \doteq \int_{\mathcal{D}} d \boldsymbol{x} g(\boldsymbol{x})$ over a domain $\mathcal{D}$ whose volume is $V \doteq \int_{\mathcal{D}} d \boldsymbol{x}$ can be interpreted as an ensemble average over a PDF $f(\boldsymbol{x})$ that is uniform over the domain: $f(\boldsymbol{x})=V^{-1}, I=V \int_{\mathcal{D}} d \boldsymbol{x} g(\boldsymbol{x}) f(\boldsymbol{x})=V\langle g\rangle$, where $\langle g\rangle \approx n^{-1} \sum_{i=1}^{n} g\left(\boldsymbol{x}_{i}\right)$, the $\boldsymbol{x}_{i}$ being sampled from $f(\boldsymbol{x})$. In statistical mechanics the prototypical application is to the computation of the ensemble average of some quantity $Q(\Gamma)$ in the canonical ensemble:

$$
\langle Q\rangle=Z^{-1} \int d \Gamma Q(\Gamma) \exp [-H(\Gamma) / T],
$$

where $Z \doteq \int d \Gamma \exp [-H(\Gamma) / T]$. The difficulties with straightforward Monte Carlo evaluation of this integral by sampling from a uniform distribution, i.e., by identifying $g(\boldsymbol{x}) \rightarrow Q(\Gamma) \exp (-H / T)$, are twofold. First, the integrand $g$ varies rapidly with $\Gamma$ and will be small for almost all random points, so a possibly prohibitively large number of points would have to be sampled to ensure accuracy. Second, the method requires the explicit numerical value of the partition function $Z$, which can be very large and difficult to evaluate.

A better procedure is to devise a way of sampling directly from the canonical distribution $P(\Gamma)=$ $Z^{-1} \exp [-H(\Gamma) / T]$; then $\langle Q\rangle \approx n^{-1} \sum_{i=1}^{n} Q\left(\boldsymbol{x}_{i}\right)$. (This is a special case of so-called importance sampling, as defined and discussed, for example, in Ref. [26].) The algorithm of $\mathrm{M}(\mathrm{RT})^{2}$ accomplishes this by defining a Markov chain that is guaranteed to converge asymptotically to $P(\Gamma)$.

In reviewing the algorithm, we follow the lucid exposition of Kalos and Whitlock [26]. The elegant technique of $\mathrm{M}(\mathrm{RT})^{2}$ is based on the fundamental ChapmanKolmogorov equation for Markov processes, which can be written for a PDF $f$ depending on a discrete timelike variable $n$ and a continuous spacelike coordinate or set of (abstract or generalized) coordinates $X$ as

$$
f_{n+1}(X)=\int d \bar{X} T(X \mid \bar{X}) f_{n}(\bar{X}) .
$$

Here $T$ is an arbitrary conditional probability density. If we introduce the transition probability $S$ according to

$$
\begin{aligned}
& T\left(X \mid X^{\prime}\right)=S\left(X \mid X^{\prime}\right) \\
& \quad+\left(1-\int d Y S(Y \mid X)\right) \delta\left(X-X^{\prime}\right),
\end{aligned}
$$

one is led to the conventional master equation [28]

$$
\begin{aligned}
f_{n+1}(X)= & \int d Y S(X \mid Y) f_{n}(Y) \\
& +\left(1-\int d Y S(Y \mid X)\right) f_{n}(X) .
\end{aligned}
$$

Here the term $S(X \mid Y)$ describes the probability of leaving the state $Y$; the parenthesized term is the conditional probability of remaining in the state. Note that $S$ is not a true conditional probability density since $\int d Y S(Y \mid X) \neq 1$.

It is easy to see that if an asymptotic distribution exists, namely $f_{n+1}=f_{n}=f$, then

$$
\int d Y S(X \mid Y) f(Y)=\int d Y S(Y \mid X) f(X) .
$$

This is satisfied by the detailed-balance condition

$$
S(X \mid Y) f(Y)=S(Y \mid X) f(X) .
$$

The $\mathrm{M}(\mathrm{RT})^{2}$ algorithm and its variants correspond to particular convenient choices of $S(X \mid Y)$.

Specifically, the algorithm proceeds by proposing a transition from state $Y$ to a new state $Y^{\prime}$ generated from 
an arbitrary conditional probability $T_{0}\left(Y^{\prime} \mid Y\right)$. The proposed state is then tested against an acceptance criterion $q$ and conditionally accepted $\left(X=Y^{\prime}\right)$ or rejected $(X=Y)$ in such a way that detailed balance is satisfied. One has $[26]$

$$
S(X \mid Y)=A(X \mid Y) T_{0}(X \mid Y),
$$

where $A$ is the acceptance probability. We will follow $\mathrm{M}(\mathrm{RT})^{2}$ in choosing

$$
A(X \mid Y)=\min (1, q(X \mid Y))
$$

where

$$
q(X \mid Y) \doteq \frac{T_{0}(Y \mid X) f(X)}{T_{0}(X \mid Y) f(Y)} .
$$

Usually an algorithm is chosen such that

$$
T_{0}(X \mid Y)=T_{0}(Y \mid X)
$$

(although we will discuss a generalization in Sec. III C 1). In that case

$$
q(X \mid Y) \rightarrow f(X) / f(Y)
$$

One can then summarize the algorithm as follows. When the given probability density at the new proposed state is larger than that at the old state $\left[q\left(Y^{\prime} \mid Y\right)>1\right]$, the new state is accepted unconditionally. Otherwise, the state is accepted with probability $q=f\left(Y^{\prime}\right) / f(Y)$. If one writes

$$
f(Y) \propto e^{-W(Y)},
$$

which is always possible for real $W$ since $f(Y)$ is a PDF and hence positive, then

$$
q=e^{-\Delta W}
$$

where $\Delta W \doteq W\left(Y^{\prime}\right)-W(Y)$; thus proposed states with lower "energy," $\Delta W<0$, are accepted unconditionally. It is easily shown that the choice (53) satisfies detailed balance, and asymptotic theorems on Markov chains guarantee that $f_{n}$ converges to $f$ for reasonable $T_{0}$.

In practice, the proposed state is usually generated by first selecting one particle randomly, then examining the consequences of changing its position by a random amount $\Delta \boldsymbol{x}=\lambda \boldsymbol{\xi}$, where the Cartesian components of $\boldsymbol{\xi}$ are sampled from a distribution uniform on $\left(-\frac{1}{2}, \frac{1}{2}\right)$ and $\lambda(\leq L)$ is a parameter that is arbitrary, in principle. That is,

$$
T_{0}(X \mid Y)= \begin{cases}\lambda^{-d} & \left(\left|x_{i}-y_{i}\right| \leq \lambda\right) \\ 0 & \text { (otherwise) }\end{cases}
$$

(The average acceptance probability and thus the rate of convergence depend on $\lambda$; see later discussion.) The effective energy $W$ is then evaluated at the proposed state $Y^{\prime}$ and the increment $\Delta W$ is computed. If $\Delta W<0$, then the proposed state is accepted as the next state in the
Markov chain. Otherwise, another random number $p$ is drawn from a distribution uniform on $[0,1)$. If $p<q$, where $q$ is defined by Eq. (58), the state is accepted $\left(X=Y^{\prime}\right)$; otherwise, the old state becomes the next state in the chain $(X=Y)$. The role of $p$ is to ensure that states with $q<1$ are accepted under a long-time average with probability $q$.

\section{Application of $\mathrm{M}(\mathrm{RT})^{2}$ to particle initialization}

The application of the $\mathrm{M}(\mathrm{RT})^{2}$ algorithm to the particle initialization problem introduces both theoretical and computational nuances.

\section{Theoretical considerations}

We will use the simple and efficient procedure described in the last paragraph of Sec. IIIB to generate a sequence of particle states $\Gamma_{i}$ (and associated Fourier amplitudes $\{\varphi\}_{i}$ ). However, because the target PDF $\mathcal{P}^{(\mathcal{M})}(\varphi)$ is couched in terms of the Fourier amplitudes, not the particle state directly, one must be cautious. In particular, although for generating particle states one may choose $X=\Gamma, f(X)=P^{(N)}(\Gamma)$, the assertion $P^{(N)}(\Gamma)=\mathcal{P}^{(\mathcal{M})}(\varphi(\Gamma))$ is not correct because it overlooks the nontrivial, nonlinear, many-to-one relation between the random variables $\Gamma$ and $\varphi$. We will now explain how to take that relation into account. For notational brevity, we will henceforth write $P^{(N)}(\Gamma) \equiv P(\Gamma)$, $\mathcal{P}^{(\mathcal{M})}(\varphi) \equiv \mathcal{P}(\varphi)$. The underlying transition probability associated with $\Gamma$ states will be written $T_{0}$, while the one associated with $\varphi$ states will be written $\mathcal{T}_{0}$.

It is important to realize that the particle states that are generated by the Markov chain have no dynamical significance. For example, they do not contain the specific pair correlations that are associated with the Coulomb interaction and that arise from the dynamical relaxation on the fast time scale during which Debye shielding is set up. There is no physical significance to the averages of arbitrary thermodynamic quantities over the $\Gamma_{i}$. Because the only information built into the calculation is (the single-time) $\mathcal{P}(\varphi)$, one is allowed to average only functions of $\varphi$ itself. Let $\mathcal{A}(\varphi)$ be an arbitrary such function. Most directly, one has

$$
\langle\mathcal{A}\rangle=\int d \varphi \mathcal{P}(\varphi) \mathcal{A}(\varphi) .
$$

Alternatively, for a compatible $P(\Gamma)$ one can write

$$
\langle\mathcal{A}\rangle=\int d \Gamma P(\Gamma) \mathcal{A}(\varphi(\Gamma)) .
$$

The fundamental constraint relating $\mathcal{P}(\varphi)$ and $P(\Gamma)$ is that

$$
\begin{aligned}
\mathcal{P}(\varphi) & =\langle\delta(\varphi-\varphi(\Gamma))\rangle \\
& =\int d \Gamma P(\Gamma) \delta(\varphi-\varphi(\Gamma))
\end{aligned}
$$


Since our only goal is to determine particle states compatible with $\mathcal{P}(\varphi)$ (but not necessarily the result of physically realizable dynamics), we have wide latitude in choosing $P(\Gamma)$. We will argue that a reasonable choice is

$$
P(\Gamma)=\frac{1}{C} \frac{\mathcal{P}(\varphi)}{\mathcal{P}_{0}(\varphi)},
$$

where $C$ is a normalizing factor (the volume of the $\Gamma$ space) and $\mathcal{P}_{0}$ is the probability density of realizing the value $\varphi$ from a uniformly distributed, statistically independent collection of particles. Note that the explicit normalization $C$ is never needed in the $\mathrm{M}(\mathrm{RT})^{2}$ algorithm.

To arrive at Eq. (63), we argue that since only $\varphi$ averages are of interest, one can choose $P(\Gamma)$ such that it depends on $\Gamma$ only through $\varphi: P(\Gamma)=F(\varphi)$ for some function $F$. In the spirit of information theory [29], this is the unique choice compatible with the lack of any further information or constraints. Without loss of generality, we can write

$$
P(\Gamma)=\mathcal{P}(\varphi) / \mathcal{Q}(\varphi),
$$

where $\mathcal{Q}(\varphi)$ is to be determined. Upon inserting the representation (64) into Eq. (62b), one obtains

$$
\begin{aligned}
\mathcal{P}(\varphi) & =\int d \Gamma \frac{\mathcal{P}(\varphi(\Gamma))}{\mathcal{Q}(\varphi(\Gamma))} \delta(\varphi-\varphi(\Gamma)) \\
& =\frac{\mathcal{P}(\varphi)}{\mathcal{Q}(\varphi)} C \int d \Gamma \frac{1}{C} \delta(\varphi-\varphi(\Gamma)),
\end{aligned}
$$

or, upon cancelling $\mathcal{P}(\varphi)$ from both sides and rearranging,

$$
\mathcal{Q}(\varphi)=C\langle\delta(\varphi-\varphi(\Gamma))\rangle_{0},
$$

where $\langle\ldots\rangle_{0}$ means the average over the $\operatorname{PDF} P_{0}(\Gamma)=$ $C^{-1}$, i.e., over a distribution of uniformly distributed, statistically independent particles. Thus $\mathcal{Q}(\varphi)=$ $C \mathcal{P}_{0}(\varphi)$ and one recovers Eq. (63).

The form (63) is a generalization of the well-known result that if $y(x)$ is a monotonically increasing function of $x$, then $P_{X}(x) d x=P_{Y}(y) d y$, or $P_{X}(x)=$ $P_{Y}(y) /|d x / d y|$. This can be written as $P_{X}(x)=$ $P_{Y}(y) / P_{0}(y)$, where $P_{0}(y)=|d x(y) / d y|$. By setting $P_{X}(x)=$ const, one sees that $P_{0}(y)$ is the PDF of $y$ associated with a uniform $x$ distribution. In the present application one may identify $x \rightarrow \Gamma, y \rightarrow \varphi$; however, one cannot simply introduce the Jacobian $\partial(\Gamma) / \partial(\varphi)$ because the relation between $\varphi$ and $\Gamma$ is not one-to-one. The form (63) reflects a particular, minimally constrained way of handling the underdeterminism.

So far we have concentrated on generating particle states $\Gamma_{i}$ that are compatible with the given $\varphi$ distribution. An alternate approach that leads one to the same PDF (63) is to directly consider a Markov chain of $\varphi$ states. Now we identify $X \rightarrow \varphi, f(X) \rightarrow \mathcal{P}(\varphi)$. One has

$$
q\left(\varphi^{\prime} \mid \varphi\right)=\frac{\mathcal{T}_{0}\left(\varphi \mid \varphi^{\prime}\right) \mathcal{P}\left(\varphi^{\prime}\right)}{\mathcal{T}_{0}\left(\varphi^{\prime} \mid \varphi\right) \mathcal{P}(\varphi)}
$$

where $\mathcal{T}_{0}\left(\varphi^{\prime} \mid \varphi\right)$ is the conditional probability of achieving $\varphi^{\prime}$, given $\varphi$, that is associated with the underlying algorithm for generating new $\Gamma$ states. The function $\mathcal{T}_{0}\left(\varphi^{\prime} \mid \varphi\right)$ is nontrivial. However, one may use the definition of conditional probability to write in complete generality

$$
\mathcal{T}_{0}\left(\varphi^{\prime} \mid \varphi\right)=\mathcal{T}_{0}\left(\varphi^{\prime}, \varphi\right) / \mathcal{P}_{0}(\varphi),
$$

where $\mathcal{P}_{0}(\varphi)$ is the $\operatorname{PDF}$ for realizing $\varphi$ at any step in the chain. The great appeal of the $\mathrm{M}(\mathrm{RT})^{2}$ method is that the joint probability $\mathcal{T}_{0}\left(\varphi^{\prime}, \varphi\right)$ need never be computed explicitly since the ratio of the $\mathcal{T}_{0}$ 's required in Eq. (54) can be written as

$$
\frac{\mathcal{T}_{0}\left(\varphi \mid \varphi^{\prime}\right)}{\mathcal{T}_{0}\left(\varphi^{\prime} \mid \varphi\right)}=\frac{\mathcal{T}_{0}\left(\varphi, \varphi^{\prime}\right) / \mathcal{P}_{0}\left(\varphi^{\prime}\right)}{\mathcal{T}_{0}\left(\varphi^{\prime}, \varphi\right) / \mathcal{P}_{0}(\varphi)}=\frac{\mathcal{P}_{0}(\varphi)}{\mathcal{P}_{0}\left(\varphi^{\prime}\right)} .
$$

Thus Eq. (67) becomes

$$
q\left(\varphi^{\prime} \mid \varphi\right)=\frac{\mathcal{P}\left(\varphi^{\prime}\right) / \mathcal{P}_{0}\left(\varphi^{\prime}\right)}{\mathcal{P}(\varphi) / \mathcal{P}_{0}(\varphi)}
$$

where all the potentials are to be computed in terms of the random particle positions.

From the point of view of generating $\varphi$ statistics, $\mathcal{P}_{0}$ is not unique; one must provide some information about how the underlying $\Gamma$ states are generated. The arguments leading to Eq. (66) show that the minimally biased choice for $\mathcal{P}_{0}$ is the PDF associated with an independent, uniform distribution of particles. This important function is considered in Appendixes B and C. There asymptotic methods are used to show that for large $N$

$$
\mathcal{P}_{0}(\varphi) \propto \exp [-N \Psi(\varphi)],
$$

where the exponent $\Psi$ satisfies

$$
\Psi(\varphi) \approx \sum_{\boldsymbol{k}}\left(\bar{\epsilon}_{\boldsymbol{k}}+\frac{1}{4} \bar{\epsilon}_{\boldsymbol{k}}^{2}\right)
$$

for $\bar{\epsilon}_{\boldsymbol{k}} \doteq\left(1+k^{2}\right)^{2}\left|\varphi_{\boldsymbol{k}}\right|^{2} \ll 1$ [see Eq. (C16)] and is determined from an implicit algorithm for larger fluctuation levels [see Eqs. (C115)]. In the limit $N \rightarrow \infty$, for which the noise level approaches $0, \bar{\epsilon}$ is very small, the quartic (in $|\varphi|$ ) correction in Eq. (72) is negligible, and one sees that the result reduces to the Gaussian approximation (35); however, for finite $N$ the result is substantially more complicated. Note that even though $\bar{\epsilon}_{\boldsymbol{k}} \ll 1$, $N \bar{\epsilon}_{\boldsymbol{k}}=O(1)$, so $\mathcal{P}_{0}$ is a nontrivial function that is not well approximated by 1 . (See Fig. 15 for a numerical confirmation of this remark.)

Although the true form of $\Psi$ is involved, its qualitative role in the Monte Carlo algorithm can be understood by considering the lowest-order result

$$
\mathcal{P}_{0}(\varphi) \propto \exp \left(-\sum_{\boldsymbol{k}} N\left(1+k^{2}\right) \widehat{E}_{\boldsymbol{k}}\right) .
$$


From the point of view of the general method of $\mathrm{M}(\mathrm{RT})^{2}$, which attempts to converge to a distribution function $f(X)$, the effective PDF in the present problem is

$$
\begin{aligned}
f(X) \propto \mathcal{P}(\varphi) / \mathcal{P}_{0}(\varphi) & \\
\approx \exp \left(-\sum_{\boldsymbol{k}}\right. & {\left[\left(\alpha+\beta k^{2}\right)\right.} \\
& \left.\left.\quad-N\left(1+k^{2}\right)\right] \widehat{E}_{\boldsymbol{k}}\right) .
\end{aligned}
$$

For the special case of uniform, independent states, for which we have shown in Sec. II C that $\alpha=\beta=N$, the lowest-order contribution from $\mathcal{P}_{0}$ cancels the $\left(\alpha+\beta k^{2}\right)$ term, leaving one with $f(X) \approx$ const and $q=1$. In this approximation all states are accepted, which demonstrates a necessary consistency: to the extent that the particle states can be considered to be Gaussian ( $N$ sufficiently large), the algorithm need "do no work."

\section{Computational algorithm}

In addition to the appearance of the reference distribution $\mathcal{P}_{0}$, the unusual elements in the present application are that the potentials are spatially nonlocal functions of the microscopic particle state $\Gamma$ and that the Fourier spectrum is resolved only to a finite $k_{\max }$ whereas the particles may occupy positions distributed continuously in $V$. Given a proposed state $\Gamma^{\prime}$, we proceed (in principle) as follows. Following standard practice in plasma particle simulations, the particles are collected onto the nearest lattice point for the purpose of computing the potentials. The resulting density distribution is (discrete-)Fouriertransformed and the potential is determined from the solution of Poisson's equation (which is trivial in Fourier space). The invariants $\widehat{E}\left(\Gamma^{\prime}\right)$ and $\widehat{\Omega}\left(\Gamma^{\prime}\right)$ are then computed, as is the change

$$
\Delta W=\left[\widehat{W}\left(\Gamma^{\prime}\right)-\widehat{W}_{0}\left(\Gamma^{\prime}\right)\right]-\left[\widehat{W}(\Gamma)-\widehat{W}_{0}(\Gamma)\right]
$$

where $\widehat{W}_{0}(\Gamma) \doteq N \Psi(\varphi(\Gamma))$ and

$$
\widehat{W}(\Gamma) \doteq \alpha \widehat{E}(\Gamma)+\beta \widehat{\Omega}(\Gamma) .
$$

Finally, the state $\Gamma^{\prime}$ is accepted or rejected according to the $\mathrm{M}(\mathrm{RT})^{2}$ criterion with $q=\exp (-\Delta W)$.

The procedure as just described is not yet optimal for machine computations since it seems to require a timeconsuming 2D Fourier transform at each step in the chain (which can be very long). That is unnecessary, however, since only one particle is moved per step and Fourier transformation is a linear operation. Because we use a nearest-grid-point algorithm to collect the particles onto the spatial lattice, it is clear that on the lattice the density of the proposed state $\Gamma^{\prime}$ will either be identical to that of $\Gamma$ or will differ from it by a deficiency of one particle at the original point $\boldsymbol{x}_{\boldsymbol{j}}$ and an excess of one particle at $\boldsymbol{x}_{\boldsymbol{j}}^{\prime}$. We can therefore calculate $\varphi_{\boldsymbol{k}}^{\prime}$ by adding to $\varphi_{\boldsymbol{k}}$ the potential associated with a test particle at $\boldsymbol{x}_{\boldsymbol{j}}^{\prime}$ and subtracting that associated with a test particle at $\boldsymbol{x}_{\boldsymbol{j}}$. The potential increment due to a test particle at $\boldsymbol{x}_{\boldsymbol{j}}$ is [cf. Eq. (40)]

$$
\varphi_{\boldsymbol{k}}^{(\boldsymbol{j})}=\left[\left(1+k^{2}\right) N\right]^{-1} \exp \left(-i \boldsymbol{k} \cdot \boldsymbol{x}_{\boldsymbol{j}}\right)
$$

thus the calculation of $\varphi_{\boldsymbol{k}}^{\prime}$ involves the computation of just two complex exponentials, or two cosines and two sines. In principle, evaluation of such quantities must be done at each step. However, run time can be decreased at the expense of memory by computing all of the possible potentials (77) once at the beginning of the run and storing them. Since there are $M_{x}\left(\frac{1}{2} M_{y}+1\right)$ independent complex Fourier modes and $M_{\text {tot }}$ lattice points, one must store approximately $Y=2\left(\frac{1}{2} M_{\text {tot }}\right)\left(M_{\text {tot }}\right)=M_{\text {tot }}^{2}$ real numbers. Usually we consider $M_{x}=2^{m}$ for reasonably small $m$, so $Y(m)=2^{4 m}$. One has, for example, $Y(3)=4 K, Y(4)=64 K, Y(5)=1 M, Y(6)=16 M$, $Y(7)=256 M$. Thus runs with $m=6$, or a $64 \times 64$ lattice, reside comfortably on desktop workstations. For the goal of testing gyrokinetic codes there is no reason to work with larger grids.

In designing a satisfactory Monte Carlo run, it is important that the ratio of acceptances to rejections be neither too small nor too large. If the ratio is small, so that almost all states are rejected, then one gains very little new information at each step, the steps are highly correlated, and the rate of convergence to the asymptotic distribution may be prohibitively slow. The same remarks pertain to the other extreme where almost all states are accepted. Common lore suggests an acceptance rate of about $50 \%$. In principle, this rate can be calculated analytically as a function of the parameters, e.g., $\{\mathcal{M}, N, \lambda, \kappa, \bar{E}\}$. Those results are somewhat tedious in detail, being expressable as infinite Fourier integrals over the characteristic function, which itself is known only as an infinite series of Bessel harmonics; our work in this area is incomplete. In practice, we proceed as follows. First we choose a temperature regime by specifying an appropriate $\kappa$. Next, we set the overall height of the target spectrum, e.g., by forcing the intensity of the longestor shortest-wavelength mode to be a specified percentage of the reference noise level. Finally, with the aid of several short trial runs we adjust $\lambda$, the size of the basic cell for the transition probability $T_{0}$, such that the rejection rate is approximately $50 \%$.

\section{EXAMPLES OF INITIALIZATION}

The preceding considerations have been implemented in a computer code whose input consists of the parameters $\{m, N, \lambda, \kappa, \bar{E}\}$ and whose output consists of a succession of particle states that sample the canonical ensemble (11).

The initial particle state is arbitrary, in principle. In addition to the uniform, independent initialization al- 
ready mentioned, we also consider the well-known Fibonacci "quiet start" defined by ([18], and references therein)

$$
\begin{aligned}
& x_{r}=\left(\frac{2 r+1}{2 N}\right) L, \\
& y_{r}=\alpha_{n-1} x_{r} \bmod L,
\end{aligned}
$$

where $r=0,1, \ldots, N-1 ; n>1$ is an arbitrary integer parameter; $\alpha_{n}$ is the $n$th Fibonacci number defined by

$$
\begin{aligned}
\alpha_{0} & =0 \\
\alpha_{1} & =1 \\
\alpha_{n} & =\alpha_{n-1}+\alpha_{n-2}
\end{aligned}
$$

and $N=\alpha_{n}$. To expedite easy comparison of the random and quiet starts, we generally choose $N$ to be a Fibonacci number. Unless we specifically state otherwise, we use $n=17(N=1597)$.

The spectrum is resolved on a lattice corresponding to $m=4\left(M_{x}=M_{y}=16\right)$. This number of modes reasonably approximates a continuum in wave-number magnitude, but is sufficiently small that desktop workstation CPU time is not exorbitant. The results presented here correspond to a box size of $L=43.3$, or $k_{\min } \approx 0.145$, $k_{\max } \approx 1.64$. These numbers are representative of other medium-size simulations of the Hasegawa-Mima and similar equations, but their precise values are not critical for the application of testing relaxation to appropriate equilibria.

We present spectral information on 2D graphs of $E_{\boldsymbol{k}}$ vs $k \doteq|\boldsymbol{k}|$. On these graphs the noise level for the special uniform, independent particle state "1" - i.e., $E_{\boldsymbol{k}, 1} \doteq$ $\left[N\left(1+k^{2}\right)\right]^{-1}$ —is shown by a dashed line; the theoretically expected spectrum, $E_{\boldsymbol{k}}=\left(\alpha+\beta k^{2}\right)^{-1}$, is shown by a solid curve. At any step in the Markov chain, the instantaneous levels $\widehat{E}_{\boldsymbol{k}}$ are indicated by a scatter plot of small plus signs. The averages of the $\widehat{E}_{\boldsymbol{k}}$ 's over the chain up to that point are indicated by a scatter plot of larger squares that in some cases is superimposed over the instantaneous data. It is convenient to measure time in units of cycles, where a cycle is defined to be $N$ steps. (On the average, each particle is moved once in the course of one cycle.)

It should be noted that if the number of particles $N$ is too small relative to the number of retained Fourier amplitudes, it may not be possible to successfully generate arbitrary Fourier spectra (consider $N=1$, for example). In such cases the Monte Carlo algorithm fails to converge, or converges to unusual spectra with $\bar{U} \neq 1$. The time dependences of $U$ and the running time average $\bar{U}$ are sensitive monitors of the convergence of the algorithm.

\section{A. The reference noise spectrum}

In the first experiment we verify that the code properly converges to the preferred noise spectrum with $\alpha=\beta=$

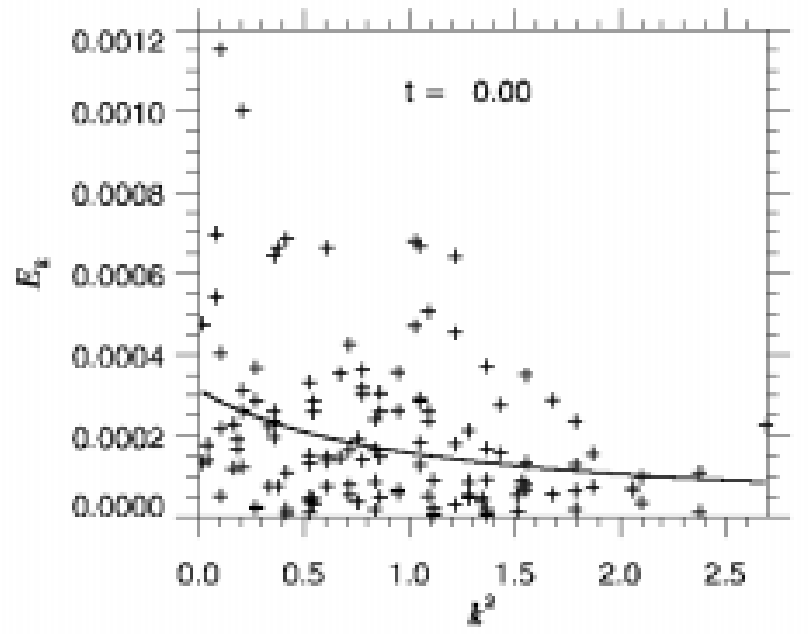

FIG. 5: Initial spectrum for a random start with $\alpha=\beta=N$ (linear ordinate).

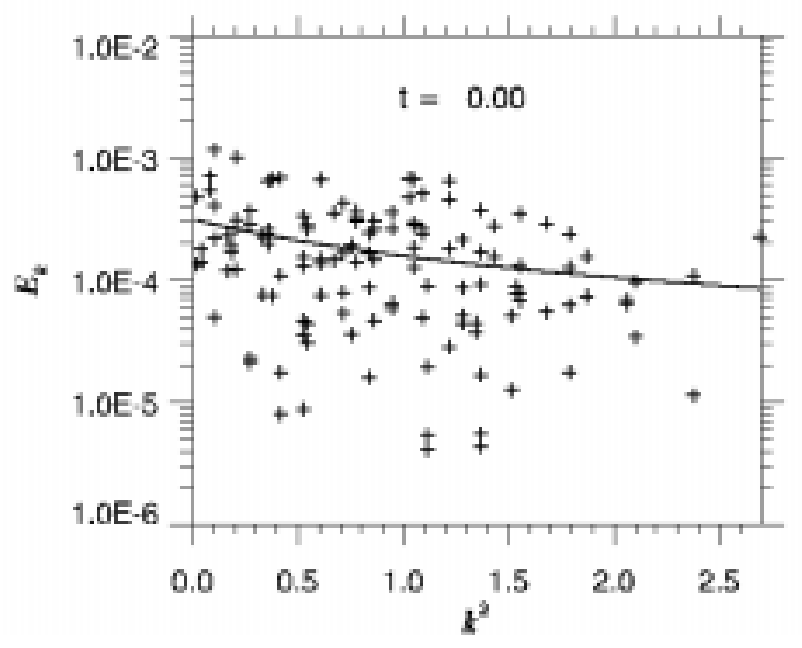

FIG. 6: Same as Fig. 5, but logarithmic ordinate.

$N$, specified by $\kappa_{1}$. (Upon referring to Table III, we see that $\kappa_{1}=6.151$ for the present parameters.) In Figs. 58 , we show the initial random Fourier intensities for both a random start (linear ordinate, Fig. 5; logarithmic ordinate, Fig. 6) and a Fibonacci start (Figs. 7 and 8). In Figs. 9-12, we show snapshots from a Fibonacci start of the Markov chain at $t \approx 1,20$, and 200. Convergence to the appropriate distribution is clearly seen, as is the expected $\sqrt{t}$ rate of convergence. The gross behavior of the instantaneous amplitudes in the final state is qualitatively similar to the initial scatter plot for the random start (Figs. 5 and 6), as it should be.

Convergence to the proper value $\bar{U}=1$ is illustrated in Figs. 13 and 14. Convergence is already recognizable from the fluctuating data in times of the order of several cycles. For this particular case, the running time average 


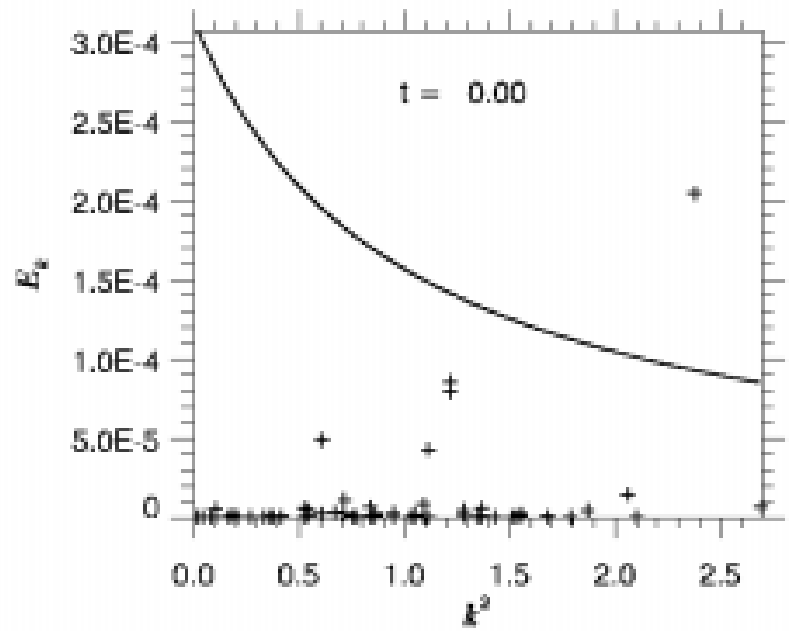

FIG. 7: Initial spectrum for a Fibonacci start with $\alpha=\beta=N$ (linear ordinate).

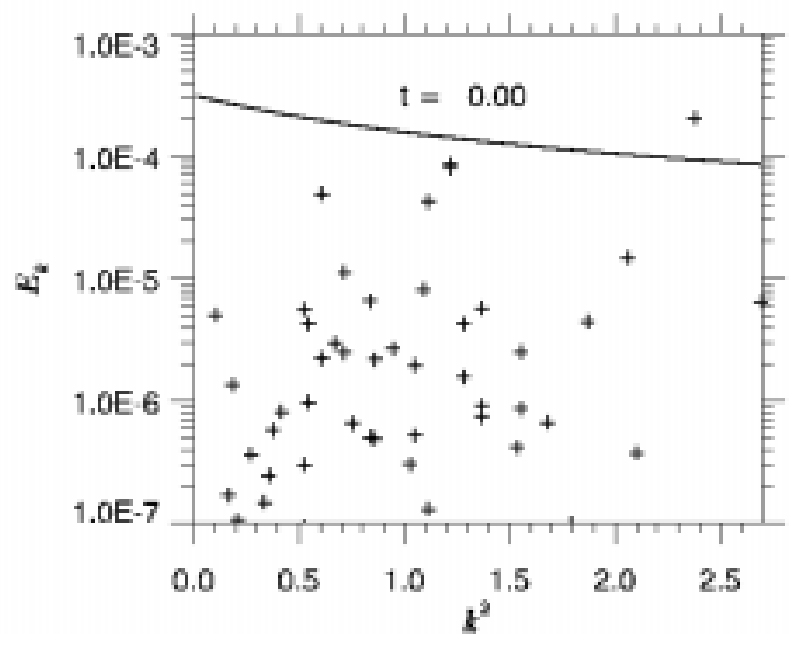

FIG. 8: Same as Fig. 7, but logarithmic ordinate.

has saturated to its correct value after about 10 cycles. Convergence within about 10 to 25 cycles is typical for the runs presented here.

Although we do not display the graphics here, it is easy to check that the particle positions have been thoroughly mixed after a small number of cycles. (Colorcoding can be used to emphasize that the particles have not just moved slightly from their initial positions.) A comparison of the final particle states with a typical set of random initial conditions shows no qualitative differences. The collected statistics verify that all states were accepted for this case, in agreement with the argument presented at the end of Sec. III C 1.

A scatter plot of the exponents $U_{0}$ for the previous run is shown in Fig. 15. Because the values are $O(1)$ but are not all equal, this figure emphasizes that $\mathcal{P}_{0}(\varphi)$ is a

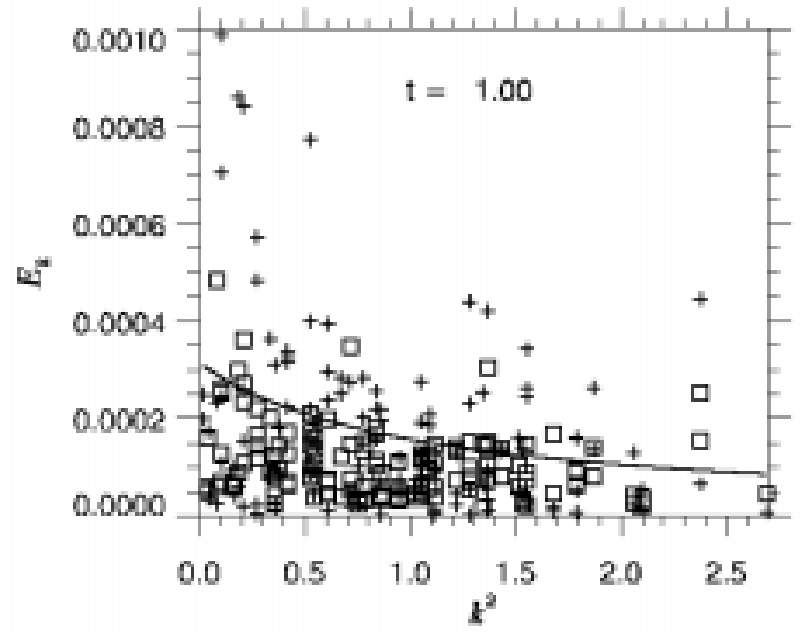

FIG. 9: Reference-case spectra for $t=1$ (linear ordinate).

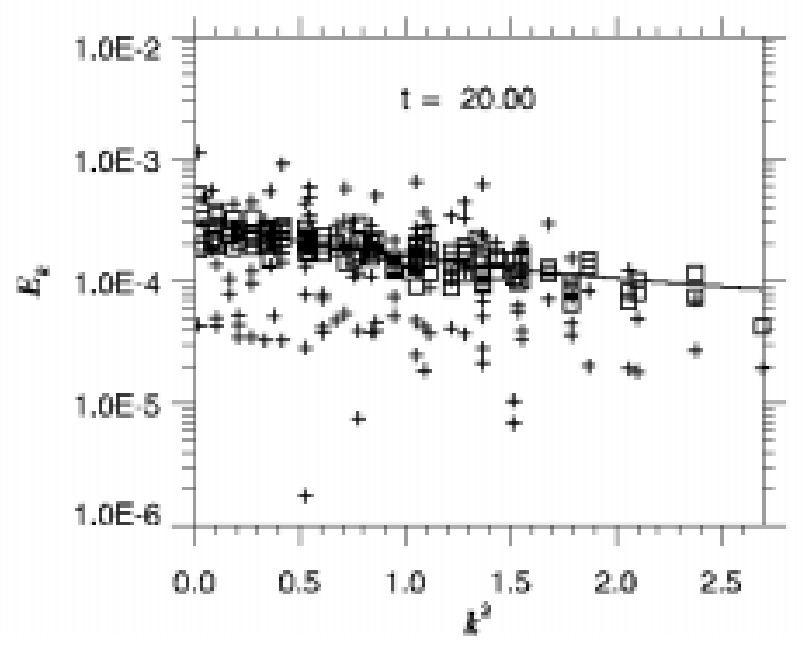

FIG. 10: Reference-case spectra for $t=20$ (logarithmic ordinate).

nontrivial function, as was remarked after Eq. (72).

In Fig. 16 we show that the algorithm has no trouble generating equal-temperature states with intensity onetenth of the reference noise level.

\section{B. Enstrophy equipartition}

Another reference case of importance is the enstrophyequipartition case $\widehat{\alpha}=0\left(\kappa=k_{a}\right)$. Although this is not a negative-temperature state (it corresponds to $\alpha=0$ or an infinite energy temperature $T_{E}$ ), it is qualitatively similar to states of negative $\alpha$ (regime I) in that the longwavelength modes are excited to relatively high levels. Working with a marginal state such as this affords a good consistency check of the software routines that relate $\alpha$, 


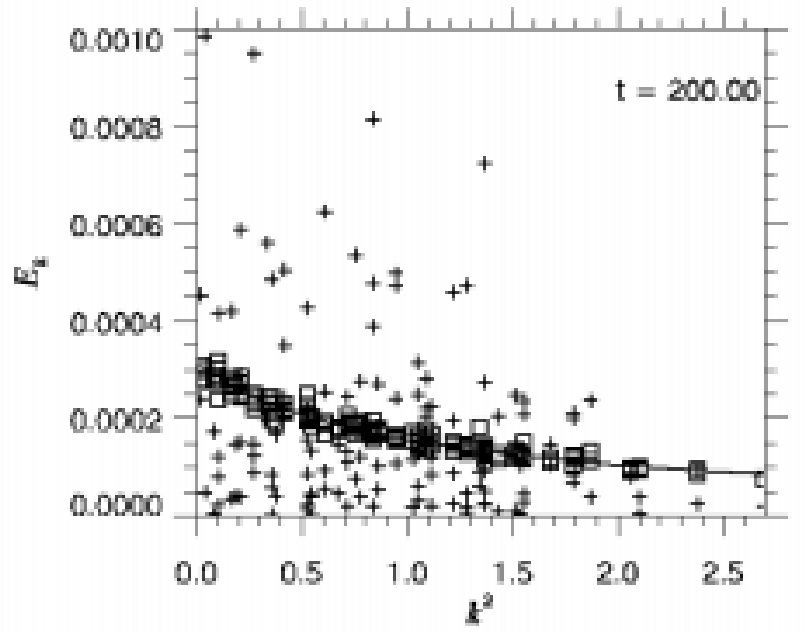

FIG. 11: Reference-case spectra for $t=200$ (linear ordinate).

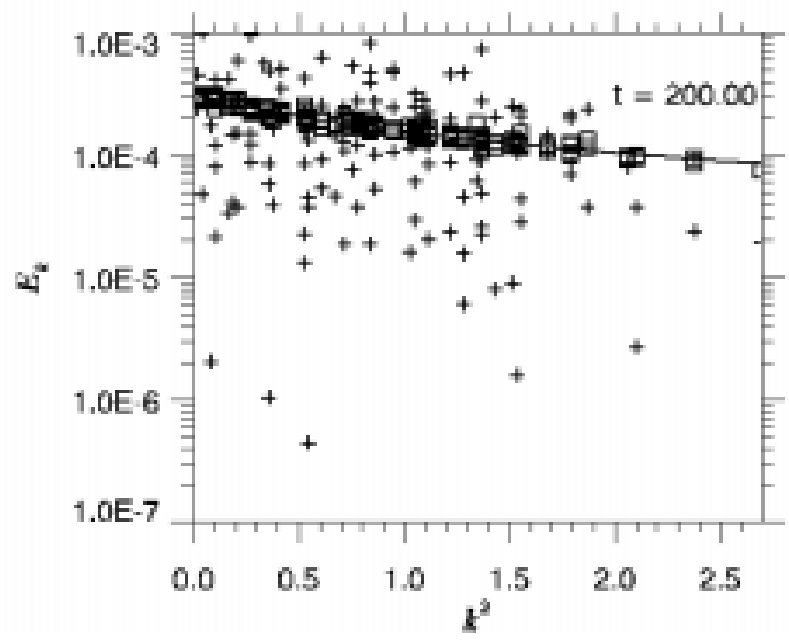

FIG. 12: Reference-case spectra for $t=200$ (logarithmic ordinate).

$\beta$, and $\kappa$. Specifying a $\kappa$ of 3.952 as indicated in Table III should lead to an $\alpha$ of 0 , as indeed it does to within numerical roundoff error. For this case, we consider two absolute levels. Define the parameter $f$ to be the ratio between the target intensity and the reference noise level for the longest-wavelength mode. We first consider $f=5$. The state after 400 cycles is shown in Figs. 17 and 18 . As one expects, convergence is dominated by the time for the longest-wavelength modes to achieve equilibrium. Note that this case demonstrates that there is no difficulty exciting some modes to a superthermal level while suppressing others to a subthermal level.

Next we consider $f=10$, thereby doubling the target intensity from the previous case. As shown in Fig. 19, this run achieves a quasi-steady state that well approximates the expected result (Fig. 20); however, after about

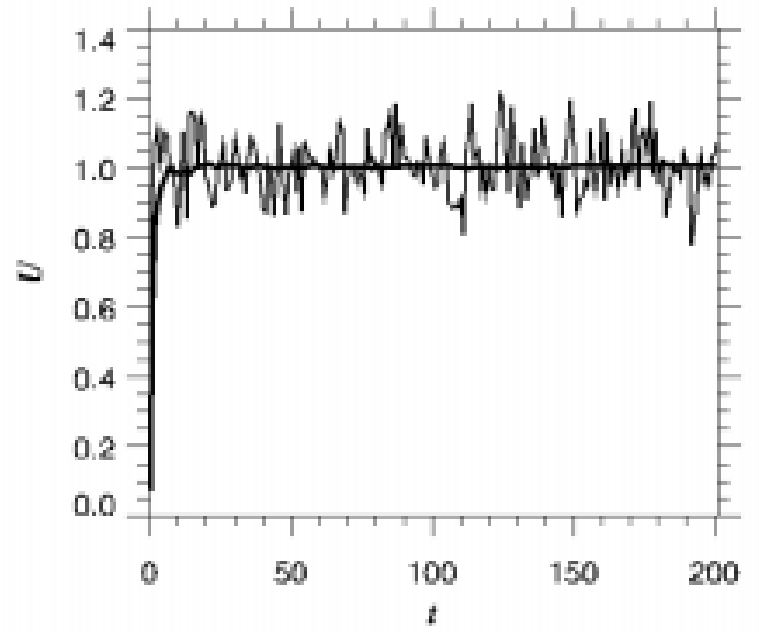

FIG. 13: Convergence to the noise state for a run with $t_{\max }=$ 200. Thin solid line, data sampled every 1.0 cycle; thick solid line; running time average.

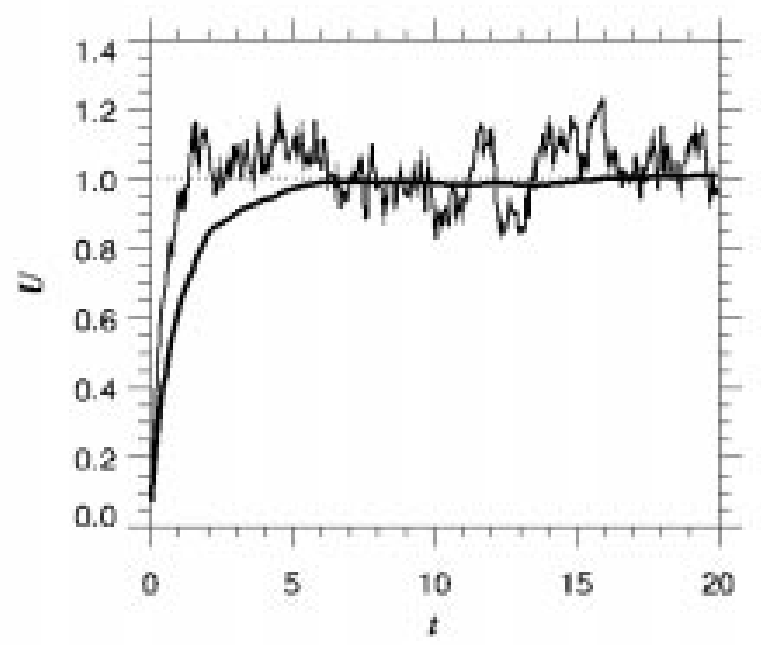

FIG. 14: Initial behavior of the convergence to the noise state. Thin solid line, data sampled every 0.05 cycles; thick solid line; running time average.

15 cycles an instability sets in. That this instability is of the longest-wavelength modes can be seen in Fig. 21.

Experience shows that such instabilities are associated with an inadequate number of particles. For lack of space, we will not show the successful result of stabilizing the present run by increasing $N$, but similar behavior is manifested by the runs presented in Secs. IV C and IV D. Note that cases with too small $N$ need not always be unstable; sometimes the chain converges, but to states with $\bar{U} \neq 1$. 


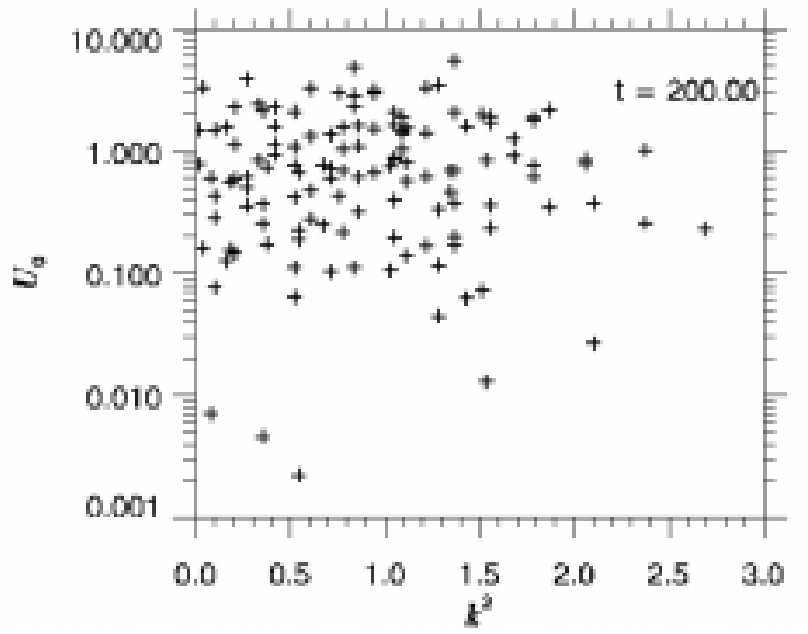

FIG. 15: Scatter plot of the exponents $U_{0}$ for the reference case plotted in Figs. 9-14.

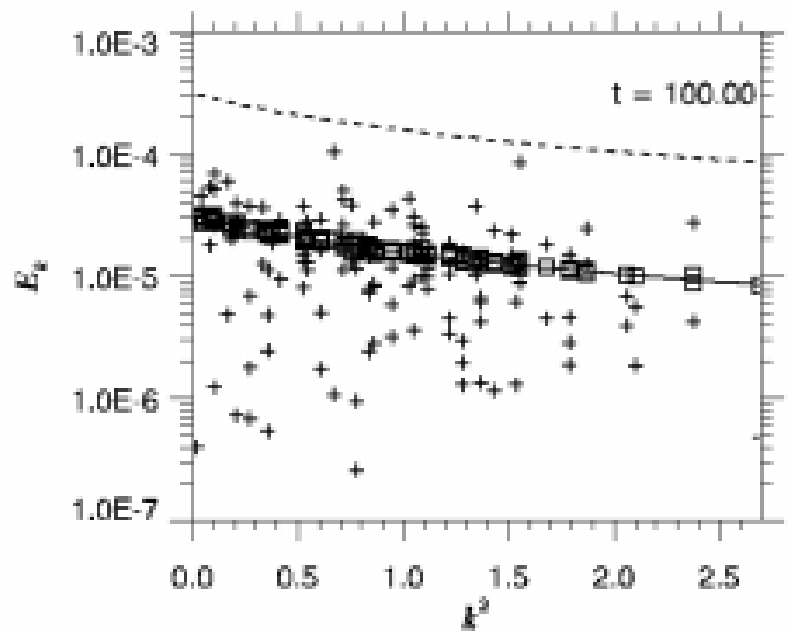

FIG. 16: Equal-temperature states with intensity one-tenth of the reference noise level (dashed line).

\section{Negative $\alpha$}

Now we generate a true negative-temperature state by somewhat arbitrarily choosing $\kappa=1.5$, a value deep in the negative- $\alpha$ regime I. Such equilibria have the longestwavelength modes excited to relatively very large levels, such as would (qualitatively) result from an inverse energy cascade. The spectrum after ten cycles is shown in Fig. 22.

\section{Negative $\beta$}

In the final experiment, we generate a state of negative $\beta$ (regime III). We arbitrarily choose $\kappa=10$. For the standard parameters $m=4, n=17$, the algorithm

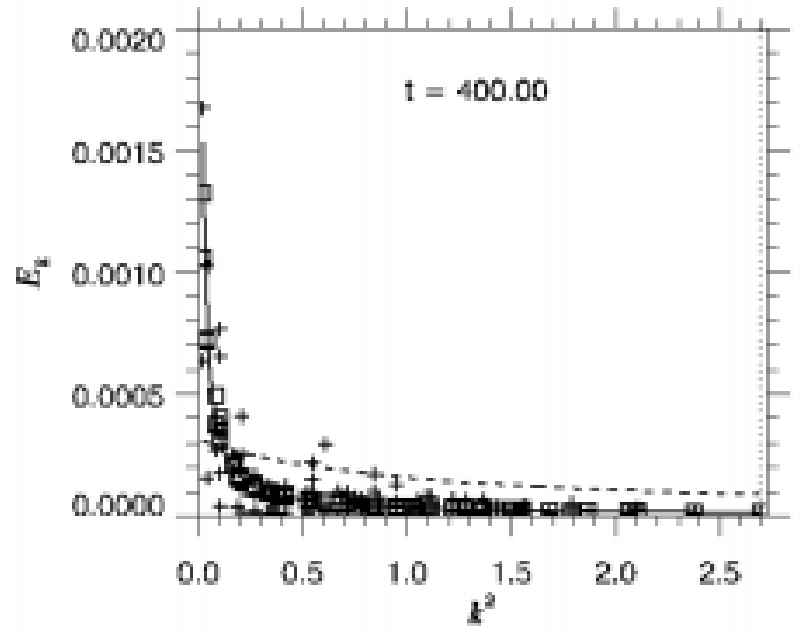

FIG. 17: Enstrophy-equipartition run $(\alpha=0)$ with $f=0.05$ (snapshot at $t=400$, linear ordinate).

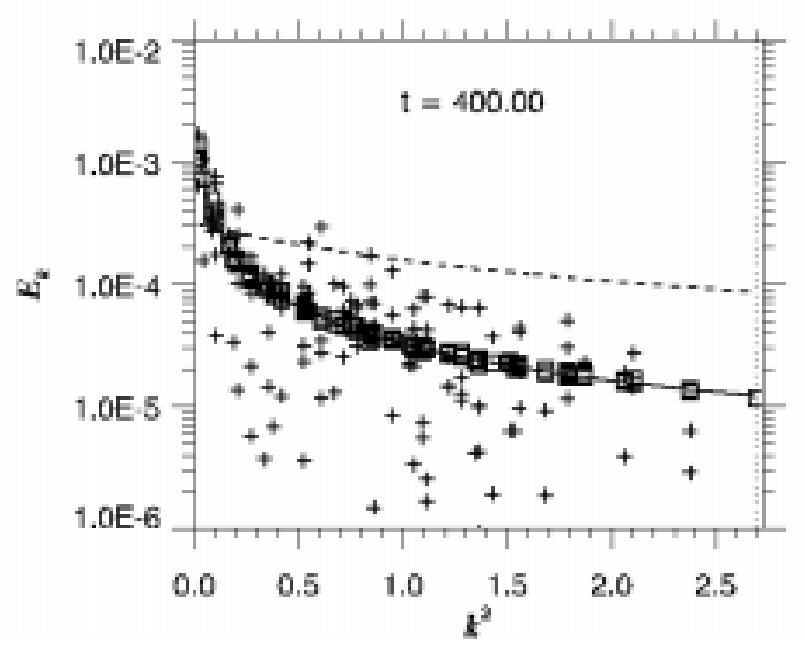

FIG. 18: Same as previous figure, but logarithmic ordinate.

appears to be well converged at $t=100$, but subsequently exhibits an instability of the shortest-wavelength mode. Increasing $n$ to 19 removes the instability; a wellconverged spectrum is shown in Fig. 23.

\section{DISCUSSION}

The calculations presented here meld two of the principal avenues to the study of nonlinear phenomena in plasma systems that exhibit strongly fluidlike behavior: (gyro)fluid simulation with Fourier amplitudes, and kinetic simulation with particles (or gyrocenters). Each has its strengths, but the two approaches should agree exactly in the inviscid, undriven limit in which collisional dissipation and the Landau resonance are ignored. Then 


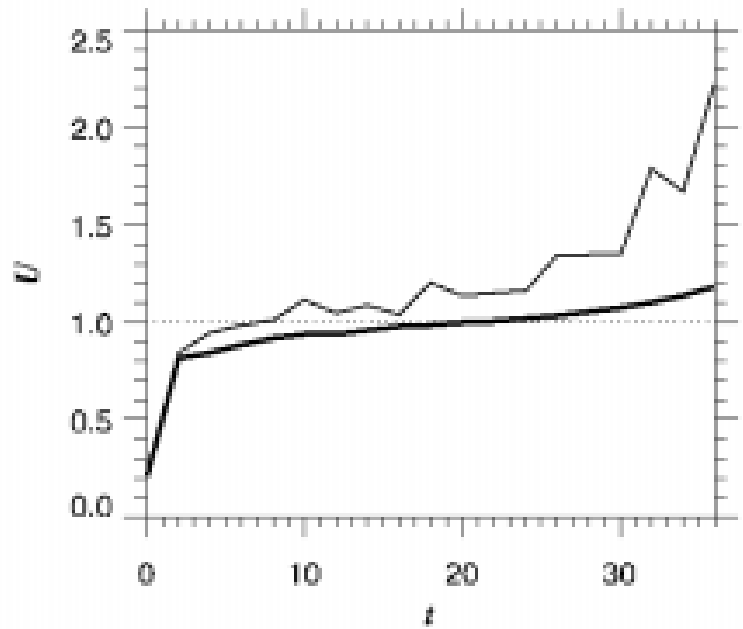

FIG. 19: Enstrophy-equipartition run with $f=0.15$, demonstrating quasi-saturation but a long-term instability.

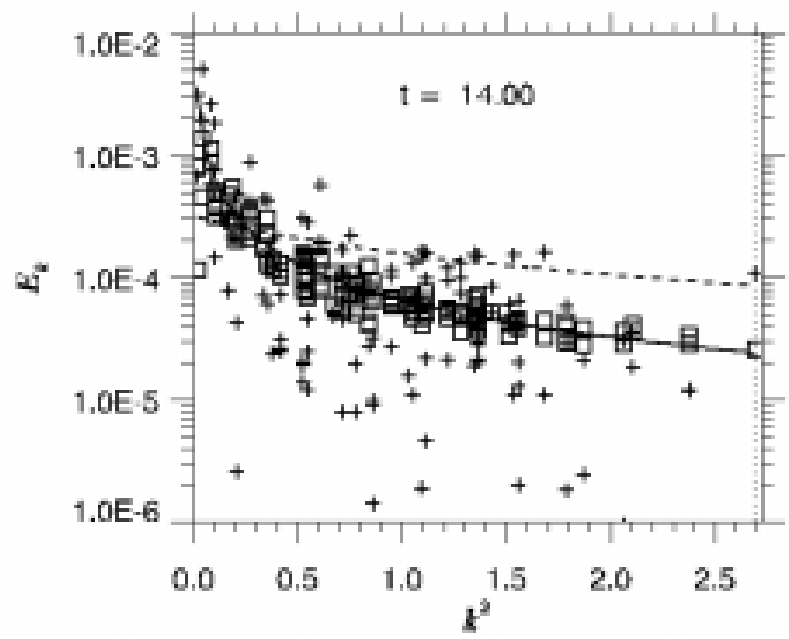

FIG. 20: Enstrophy-equipartition spectra in the quasisaturated regime of Fig. 19.

the spectrum predicted by either approach should nonlinearly relax to the prediction of the appropriate canonical ensemble. The Hasegawa-Mima equation is arguably the simplest nonlinear equation with relevance to magnetized plasma physics. Nevertheless, its canonical behavior (for truncated Fourier spectra) is entirely nontrivial, including the existence of negative-temperature states. In this paper we showed how to construct particle realizations compatible with those Fourier spectra by using a generalization of the well-known Monte Carlo algorithm of Metropolis et al. [19]. The numerical aspects of the calculation are straightforward. However, the calculation of the nontrivial PDF $P_{0}(\varphi)$ (the probability density of achieving the set of Fourier amplitudes $\{\varphi\}$ from a uniform distribution of statistically independent gyrocen-

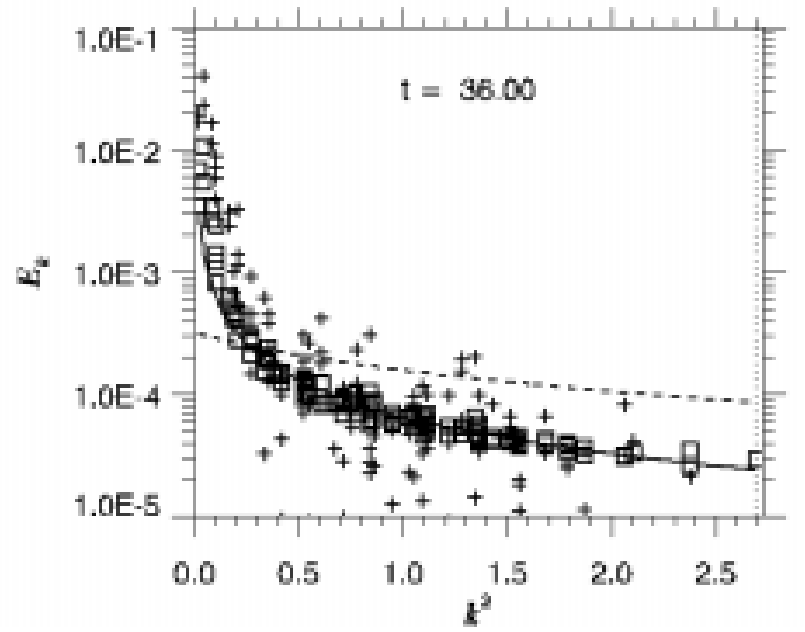

FIG. 21: Long-time spectra for the unstable case corresponding to Fig. 19.

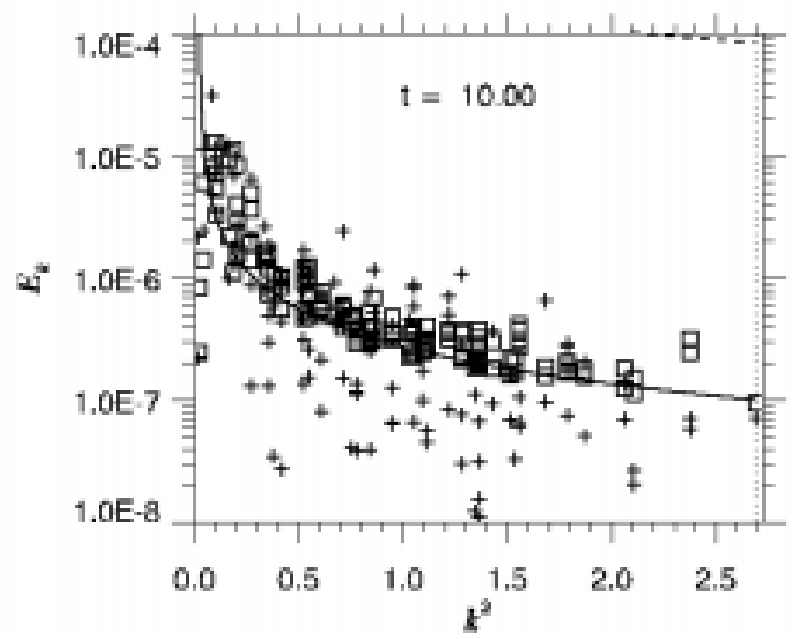

FIG. 22: A state of negative $\alpha . \quad \kappa=1.5 ; f=10 ; n=19$ $(N=4181)$.

ters) presents an interesting problem in asymptotics, as described in Appendix C.

\section{A. Application to particle simulations}

In the proposed application to particle simulations, issues arise concerning both the interpretation of averaging procedures as well as the practical implementation of the required fluid limit.

\section{Averaging procedures}

We first consider the interpretation of averages over the chain of states. In particular, we pose the following 


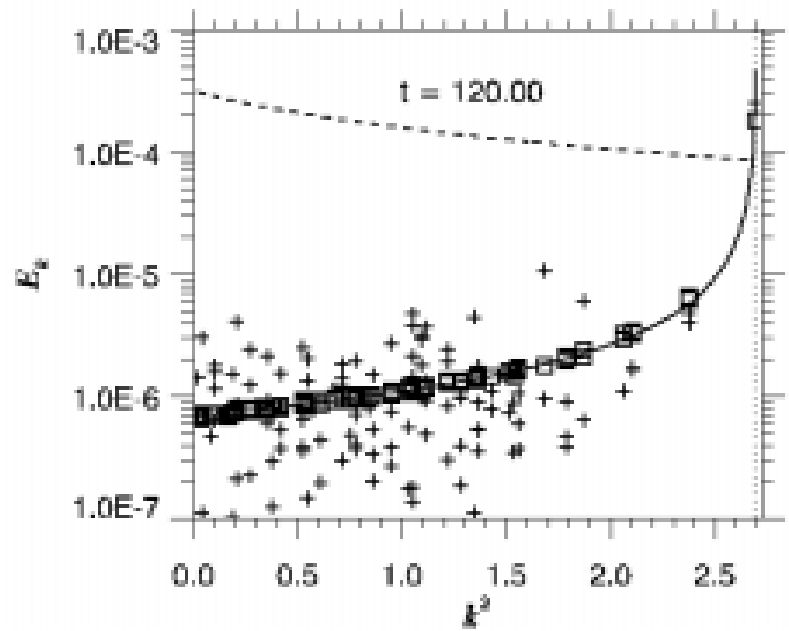

FIG. 23: A state of negative $\beta . \kappa=10 ; n=19(N=4181)$.

questions: (i) What is the role of a single microstate? (ii) Does an ensemble average yield any additional information?

To answer these questions, one must be precise about the distinction between time and ensemble averages. Let $r$ label one of $R$ realizations, each initialized by a random sample drawn from the canonical distribution with specified $E$ and $\Omega$. Of course, $\widehat{E}_{\boldsymbol{k}}^{(r)}(t=0) \doteq \widetilde{E}_{\boldsymbol{k}}^{(r)} \neq E_{\boldsymbol{k}}$. Each realization will evolve conserving its own $\widehat{E}=\widetilde{E}$ and $\widehat{\Omega}$, and one expects that the action of the nonlinear terms will (on the average) readjust $\widehat{E}_{\boldsymbol{k}}(t)$ to the canonical spectrum corresponding to $\widehat{E}$ (not $E)$. Within one realization, the only sensible average to perform is the time average; one expects

$$
\begin{aligned}
& \overline{E_{\boldsymbol{k}}^{(r)}(t)}=\widetilde{E}_{\boldsymbol{k}}^{(r)}, \\
& \overline{\widehat{E}^{(r)}(t)}=\widetilde{E}^{(r)} .
\end{aligned}
$$

Demonstration of correct time-averaged relaxation to a variety of positive- and negative-temperature canonical states is probably the most stringent test that can be performed on nonlinear simulation modules. Note that it is unnecessary to perform a new Monte Carlo run before each test of a simulation code or sequence of code updates. A few representative cases can be computed once and stored in disk files; they can then be used repeatedly in relaxation tests. Although those will be restricted in the numbers of particles $N$ and Fourier amplitudes $\mathcal{M}$, that is probably not crucial because it is difficult (though not impossible) to imagine software bugs that would not show up with a randomly chosen $N$ and $\mathcal{M}$.

In order to demonstrate convergence to the specified $E$, a true ensemble average must be performed:

$$
E=\langle\widetilde{E}\rangle \approx \frac{1}{R} \sum_{r=1}^{R} \widetilde{E}^{(r)} .
$$

To reduce the variance of this calculation, one should not identify realizations with successive states along the Markov chain but rather with states separated by at least one correlation length. It is unlikely that such an experiment will be performed routinely because it involves many simulation runs and it is improbable that a bug causing erroneous convergence at this stage would not have been caught by the tests on individual realizations.

\section{Practical considerations}

The present paper presents the theory of generating particle microstates compatible with the nontrivial thermal equilibria of particular nonlinear fluid equations. Of course, a complete particle simulation does not approximately integrate a fluid equation, but rather a nonequilibrium kinetic equation. Therefore, achieving the inviscid, undriven fluid limit may not be trivial in practice.

In general, the physics contained in kinetic equations includes wave-particle interactions (Landau damping). To achieve the fluid limit, the Landau resonance must be turned off by setting the parallel wave number $k_{\|}$to zero. That is easy in unsheared slab geometry but not necessarily trivial in the presence of magnetic shear, which must be set to zero.

If one is to achieve the Hasegawa-Mima limit, one must enforce adiabatic electrons and $T_{i}=0$. Strictly speaking, adiabatic electron response is not compatible with $k_{\|}=$ 0 , so one cannot merely employ a two-species code with $k_{\|}=0$. Rather, the electron response must be built into the gyrokinetic Poisson equation (as in Appendix A), and only the ions should be integrated explicitly. Usually those ions are initialized by sampling from a Maxwellian velocity distribution. Setting $T_{i}=0$ in that distribution may cause problems; however, it should be adequate to simply use a small but nonzero ratio of $T_{i} / T_{e}$.

One should also note that thermal-equilibrium spectra apply only to homogeneous simulations (with, of course, no macroscopic linear drive or damping). Turning off linear drive is easy in two-scale formulations that incorporate the effects of background profile variations into constant or slowly varying parameters; it may be more difficult for global simulations with nonperiodic boundary conditions. Note that such simulations are not homogeneous.

Finally, in modern simulation practice the full gyrokinetic equation is not solved directly. Rather, the socalled $\delta f$ algorithm is employed, in which only the deviation $\delta f$ from a Maxwellian is integrated explicitly (by the method of characteristics). A description of that intrinsically low-noise method, references to the original work, and some theoretical discussion of sampling noise can be found in Ref. [30]. Although the basic method would seem to be well suited to simulations of thermalequilibrium fluid noise, the long-time behavior of $\delta f$ simulations may be unstable in the collisionless limit (which is required for the Hasegawa-Mima spectra discussed in 
the present paper). The basic problem with that limit (unbounded increase of an entropylike function) was elucidated in Ref. [31], and a possible solution (involving the use of a numerical "thermostat") was advanced in Ref. [6]. However, further research on both theory and technique is required; therefore, we leave the demonstration of collisionless Hasegawa-Mima thermal-equilibrium spectra in a $\delta f$ simulation to future work.

\section{B. Final remarks}

Although our original motivation was the flexible initialization and robust testing of gyrokinetic simulation codes, the physics and algorithms we have discussed may be of more general interest. The $\delta f$ algorithm mentioned in Sec. VA 2 is itself at core a Monte Carlo sampling technique [30] that can be used for integrating a variety of continuous partial differential equations, possibly unrelated to plasma physics. Gyrocenter motion has much in common with 2D turbulence as well as the dynamics of point vortices moving in two dimensions [32], which can be treated both with particle-simulation techniques [33, 34], fluid approaches [35, and references therein], and specially designed laboratory experiments $[36$, and references therein]. Finally, the way of handling the many-toone relation between the particles and the Fourier amplitudes, as well as the statistics issues surrounding the determination of the basic PDF $P_{0}(\varphi)$, may be of interest in a variety of contexts, including the use of maximumentropy methods in pattern recognition [37].

\section{Acknowledgments}

We are grateful to W. W. Lee and J. Reynders for many informative discussions about the theory and numerical implementation of gyrokinetics.

This work was supported by U. S. Department of Energy Contract No. DE-AC02-76-CHO-3073.

\section{APPENDIX A: GYROKINETIC DERIVATION OF THE HASEGAWA-MIMA EQUATION}

In an attempt to make the manuscript reasonably self-contained, we present here a brief derivation of the Hasegawa-Mima equation using the gyrokinetic formalism [9]. Further details and discussion of the equation can be found in the original references $[11,22]$, in Bowman's dissertation [23], and in Ref. [38].

In a set of dimensionless variables in which density is normalized to the mean density $\bar{n}$, lengths are normalized to the "sound radius" $\rho_{\mathrm{s}} \doteq c_{\mathrm{s}} / \omega_{c i}$ [where $c_{\mathrm{s}}$ is the sound speed $\left(T_{e} / m_{i}\right)^{1 / 2}$ and $\omega_{c i}$ is the ion gyrofrequency $\left.q_{i} B / m_{i} c\right]$, times are normalized to $L_{n} / c_{\mathrm{s}}$, where $L_{n}$ is the density scale length, and the electrostatic poten- tial is normalized to $\left(T_{e} / e\right)\left(\rho_{\mathrm{s}} / L_{n}\right)$, the continuity equation for the perturbed ion density is

$$
\frac{\partial n_{i}}{\partial t}+V_{*} \frac{\partial \varphi}{\partial y}+\boldsymbol{V}_{E} \cdot \nabla n_{i}=0
$$

Here the diamagnetic velocity $V_{*} \doteq\left(c T_{e} / e B\right) L_{n}^{-1}$ is unity in the present units, but is written symbolically for emphasis; the $\boldsymbol{E} \times \boldsymbol{B}$ velocity is $\boldsymbol{V}_{E} \doteq c \widehat{\boldsymbol{z}} \times \boldsymbol{\nabla} \varphi / B$. The potential is determined by the quasineutrality condition, appropriate for low-frequency, long-wavelength fluctuations:

$$
\nabla_{\perp}^{2} \varphi=-\left(n_{i}^{G}-n_{e}^{G}\right) .
$$

Here $n_{i}^{G}$ and $n_{e}^{G}$ are the gyrocenter densities; one has $n_{e}^{G} \approx n_{e}$ because the electron gyroradius is very small. The Laplacian term describes the ion polarization charge density $\rho^{\text {pol }}$. [The conventional Laplacian in Poisson's original equation is $O\left(\lambda_{D}^{2} / \rho_{\mathrm{s}}^{2}\right)$; this is small in the gyrokinetic ordering [15] and is neglected in the approximation of quasineutrality.] This is defined by the continuity equation

$$
\partial_{t} \rho^{\mathrm{pol}}+\nabla \cdot \boldsymbol{j}^{\mathrm{pol}}=0,
$$

where the ion polarization current is $\boldsymbol{j}^{\text {pol }}=n_{i} q_{i} \boldsymbol{V}^{\text {pol }}$, with [39]

$$
\boldsymbol{V}^{\mathrm{pol}}=\frac{1}{\omega_{c i}} \frac{\partial}{\partial t}\left(\frac{c \boldsymbol{E}_{\perp}}{B}\right) .
$$

In the approximation of Hasegawa and Mima, the electron response is assumed to be adiabatic ${ }^{6}$ :

$$
n_{e}=\varphi
$$

One then obtains the simplest form of the gyrokinetic Poisson equation:

$$
\left(1-\nabla_{\perp}^{2}\right) \varphi=n_{i}^{G} .
$$

If one substitutes this expression for $n_{i}$ into the continuity equation (A1), one is led immediately to Eq. (5) of the text.

When the more realistic case of nonadiabatic electron response is considered, both the linear and nonlinear terms are modified. The resulting equation is called the Terry-Horton equation [22]. It conserves just one invariant $\left[\left\langle\left(\delta n_{i}^{G}\right)^{2}\right\rangle\right.$, essentially the sum of the energy and enstrophy], so its equilibrium statistical mechanics differs from that of the Hasegawa-Mima equation and, in fact, is quite nontrivial. Nevertheless, we feel that our

\footnotetext{
${ }^{6}$ In the more physically reasonable generalized Hasegawa-Mima equation, electron response is taken to vanish for $k_{\|}=0$ modes. Further references to that model and aspects of the resulting dynamics were discussed by J. A. Krommes and C.-B. Kim, Phys. Rev. E 62, 8508 (2000).
} 
fundamental concerns of testing gyrokinetic simulations and exploring the generation of two-temperature equilibria are better served by concentrating on the simpler Hasegawa-Mima equation, so we do not consider the Terry-Horton equation further in this work.

\section{APPENDIX B: CALCULATION OF THE FUNDAMENTAL PROBABILITY DENSITY FUNCTION $\mathcal{P}_{0}(\varphi)$}

In this appendix we will use the notation $\varphi$ instead of $\varphi$ to indicate the set of Fourier amplitudes (more specifically, the set of the real and imaginary parts of each $\varphi_{\boldsymbol{k}}$ ). The discussion in Sec. III shows that the quantity $\mathcal{P}_{0}(\boldsymbol{\varphi})$ plays a crucial role in the proposed Monte Carlo method. Again, $\mathcal{P}_{0}(\varphi)$ is the PDF for the Fourier amplitudes of the potentials arising from an ensemble of independent gyrocenters, each of which is distributed uniformly.

In the calculations to follow, we will prefix formula numbers from Abramowitz and Stegun [40] with AS, and prefix ones from Gradshteyn and Ryzhik [41] with GR.

\section{General expression}

To compute $\mathcal{P}_{0}$, one may recall the standard result, written first for a single real random variable $\widetilde{\psi}$, that

$$
P(\psi)=\langle\delta(\psi-\widetilde{\psi})\rangle
$$

Of course, when the $\langle\ldots\rangle$ average is expressed in terms of $P(\psi)$ itself, Eq. (B1) is a tautology. However, when the random properties of $\widetilde{\psi}$ are expressed in terms of another underlying variable $X \equiv \widetilde{x}$ whose density is $P_{X}(x)$, Eq. (B1) is nontrivial:

$$
P(\psi)=\int d x P_{X}(x) \delta(\psi-\widetilde{\psi}(x))
$$

It is often convenient to work with the Fourier transform of this result, i.e., to compute the characteristic function

$$
P_{k}=\int d \psi e^{-i k \psi} P(\psi)=\langle\exp [-i k \widetilde{\psi}(\widetilde{x})]\rangle
$$

This average is analytically tractable if the relationship between $\widetilde{\psi}$ and $\widetilde{x}$ is sufficiently simple.

To apply this procedure to the present problem, we write formally

$$
\mathcal{P}_{0}(\varphi)=\langle\delta(\varphi-\tilde{\varphi})\rangle
$$

where the ensemble average is to be taken over the ensemble of independent, uniformly distributed gyrocen- ters. More explicitly,

$$
\begin{aligned}
\mathcal{P}_{0}(\boldsymbol{\varphi})= & \left\langle\prod_{m=1}^{\mathcal{M}} \delta\left(\varphi_{m}^{(r)}-\widetilde{\varphi}_{m}^{(r)}\right) \delta\left(\varphi_{m}^{(i)}-\widetilde{\varphi}_{m}^{(i)}\right)\right\rangle \\
= & \int \frac{d \boldsymbol{p}_{1}}{(2 \pi)^{2}} \frac{d \boldsymbol{p}_{2}}{(2 \pi)^{2}} \cdots \frac{d \boldsymbol{p}_{\mathcal{M}}}{(2 \pi)^{2}} \\
& \times \prod_{m=1}^{\mathcal{M}} e^{i \boldsymbol{p}_{m} \cdot \boldsymbol{\varphi}_{m}}\left\langle e^{-i \boldsymbol{p}_{m} \cdot \widetilde{\boldsymbol{\varphi}}_{m}}\right\rangle
\end{aligned}
$$

where

$$
\boldsymbol{p}_{m} \doteq\left(\begin{array}{c}
p_{m}^{(r)} \\
p_{m}^{(i)}
\end{array}\right), \quad \boldsymbol{\varphi}_{m} \doteq\left(\begin{array}{c}
\varphi_{m}^{(r)} \\
\varphi_{m}^{(i)}
\end{array}\right)
$$

and $m$ ranges over all $\mathcal{M}$ Fourier modes: $\boldsymbol{\varphi}_{m} \equiv \boldsymbol{\varphi}_{\boldsymbol{k}_{m}}$. If one defines, for any $\boldsymbol{k}$,

$$
a_{\boldsymbol{k}} \doteq\left[\left(1+k^{2}\right) N\right]^{-1}
$$

then the gyrokinetic Poisson equation that relates the potentials to the random gyrocenter positions is, from Eq. (40),

$$
\widetilde{\varphi}_{\boldsymbol{k}}=a_{\boldsymbol{k}} \sum_{j=1}^{N} e^{-i \boldsymbol{k} \cdot \widetilde{\boldsymbol{x}}^{(j)}}
$$

One thus has

$$
\begin{aligned}
e^{-i \boldsymbol{p} \cdot \widetilde{\boldsymbol{\varphi}}}= & \exp \left(-i a_{\boldsymbol{k}} p^{(r)} \sum_{j=1}^{N} \cos \left(\boldsymbol{k} \cdot \widetilde{\boldsymbol{x}}^{(j)}\right)\right) \\
& \times \exp \left(i a_{\boldsymbol{k}} p^{(i)} \sum_{l=1}^{N} \sin \left(\boldsymbol{k} \cdot \widetilde{\boldsymbol{x}}^{(l)}\right)\right)
\end{aligned}
$$

or

$$
e^{-i \boldsymbol{p}_{m} \cdot \widetilde{\boldsymbol{\varphi}}_{m}}=\prod_{j=1}^{N} \mathcal{R}_{m}^{(j)}\left(w_{m}^{(r)}\right) \mathcal{I}_{m}^{(j)}\left(w_{m}^{(i)}\right)
$$

where $\boldsymbol{w} \doteq a \boldsymbol{p}$ and

$$
\begin{aligned}
\mathcal{R}_{m}^{(j)}\left(w_{m}^{(r)}\right) \doteq & \sum_{n_{r}=-\infty}^{\infty} J_{n_{r}}\left(w_{m}^{(r)}\right) e^{-i n_{r} \pi / 2} \\
& \times e^{i n_{r} \boldsymbol{k}_{m} \cdot \widetilde{\boldsymbol{x}}^{(j)}} \\
\mathcal{I}_{m}^{(j)}\left(w_{m}^{(i)}\right) \doteq & \sum_{n_{i}=-\infty}^{\infty} J_{n_{i}}\left(w_{m}^{(i)}\right) e^{i n_{i} \boldsymbol{k}_{m} \cdot \widetilde{\boldsymbol{x}}^{(j)}}
\end{aligned}
$$

Thus the Fourier transform of $\mathcal{P}_{0}(\varphi)$ is

$$
\mathcal{P}_{0}\left(\boldsymbol{p}_{1}, \ldots, \boldsymbol{p}_{m}\right)=\left\langle\prod_{m=1}^{\mathcal{M}} \prod_{j=1}^{N} \mathcal{R}_{m}^{(j)}\left(w_{m}^{(r)}\right) \mathcal{I}_{m}^{(j)}\left(w_{m}^{(i)}\right)\right\rangle .
$$

Note that in general the ensemble average does not commute with the product symbols. 
To simplify Eq. (B12), let us first recall that the gyrocenters are independent. Therefore

$$
\mathcal{P}_{0}\left(\boldsymbol{p}_{1}, \ldots, \boldsymbol{p}_{m}\right)=\prod_{j=1}^{N}\left\langle\prod_{m=1}^{\mathcal{M}} \mathcal{R}_{m}^{(j)}\left(w_{m}^{(r)}\right) \mathcal{I}_{m}^{(j)}\left(w_{m}^{(i)}\right)\right\rangle .
$$

It is not hard to see that for uniformly distributed gyrocenters the Fourier amplitudes are independent. Then

$$
\mathcal{P}_{0}\left(\boldsymbol{p}_{1}, \ldots, \boldsymbol{p}_{m}\right)=\prod_{m=1}^{\mathcal{M}} P_{0}\left(\boldsymbol{p}_{m}\right)
$$

where

$$
\begin{aligned}
P_{0}(\boldsymbol{p}) & \doteq \prod_{j=1}^{N}\left\langle\mathcal{R}^{(j)}\left(w^{(r)}\right) \mathcal{I}^{(j)}\left(w^{(i)}\right)\right\rangle \\
& =\left\langle\mathcal{R}^{(1)}\left(w^{(r)}\right) \mathcal{I}^{(1)}\left(w^{(i)}\right)\right\rangle^{N}
\end{aligned}
$$

since each gyrocenter has identical statistics. One has, for any gyrocenter,

$$
\begin{aligned}
\left\langle\mathcal{R}\left(w^{(r)}\right) \mathcal{I}\left(w^{(i)}\right)\right\rangle & =\sum_{n_{r}, n_{i}} J_{n_{r}}\left(w^{(r)}\right) e^{-i n_{r} \pi / 2} J_{n_{i}}\left(w^{(i)}\right) \\
& \times\left\langle\exp \left[i\left(n_{r}+n_{i}\right) \boldsymbol{k} \cdot \widetilde{\boldsymbol{x}}\right]\right\rangle \quad(\mathrm{B} 16 \mathrm{a}) \\
& =\sum_{n} J_{n}\left(w^{(r)}\right) e^{i n \pi / 2} J_{n}\left(w^{(i)}\right)(\mathrm{B} 16 \mathrm{~b}) \\
& =J_{0}(w),
\end{aligned}
$$

where in the last step we employed Graf's addition theorem (AS 9.1.79). Finally, then,

$$
\mathcal{P}_{0}(\varphi)=\prod_{m=1}^{\mathcal{M}} P_{0}\left(\varphi_{m}\right)
$$

where (now writing $\boldsymbol{\varphi}$ instead of $\boldsymbol{\varphi}_{m}$ for convenience)

$$
\begin{aligned}
P_{0}(\boldsymbol{\varphi}) & \doteq \int \frac{d \boldsymbol{p}}{(2 \pi)^{2}} e^{i \boldsymbol{p} \cdot \boldsymbol{\varphi}} J_{0}^{N}(a p) \\
& =\frac{1}{2 \pi} \int_{0}^{\infty} p d p J_{0}(|\varphi| p) J_{0}^{N}(a p) .
\end{aligned}
$$

Note that $P_{0}(\varphi)$ is the PDF for the two-component vector $\varphi$ of real and imaginary parts; it is normalized such that $\int d \varphi P_{0}(\boldsymbol{\varphi})=\int d \varphi^{(r)} d \varphi^{(i)} P_{0}(\boldsymbol{\varphi})=1$. However, by symmetry the result will depend only on $\varphi=|\varphi|$, so we will frequently quote the magnitude $\operatorname{PDF} P_{0}(\varphi)$, where $P_{0}(\varphi)=2 \pi \varphi P_{0}(\varphi)$. If one changes variables to

$$
\bar{p} \doteq p /\left(1+k^{2}\right)
$$

and defines

$$
\bar{\varphi} \doteq\left(1+k^{2}\right) \varphi
$$

$\left(\bar{\varphi} \equiv n_{i}^{G}\right)$, then one obtains the final result

$$
\begin{array}{r}
\bar{P}_{0}(\bar{\varphi} ; N)=(2 \pi \bar{\varphi}) \bar{P}_{0}(\bar{\varphi} ; N), \\
\bar{P}_{0}(\bar{\varphi} ; N) \doteq \int_{0}^{\infty} \bar{p} d \bar{p} J_{0}(|\bar{\varphi}| \bar{p}) J_{0}^{N}(\bar{p} / N) .
\end{array}
$$

It is useful to note that the result (B21b) may also be written in the interesting form

$$
\bar{P}_{0}(\overline{\boldsymbol{\varphi}} ; N)=\left\langle\delta\left(\overline{\boldsymbol{\varphi}}+\sum_{j=1}^{N} \overline{\boldsymbol{k}}_{j}\right)\right\rangle_{\overline{\boldsymbol{k}}},
$$

where the $\overline{\boldsymbol{k}}$ 's are effective wavevectors (not to be confused with the $\boldsymbol{k}$ labels of the Fourier amplitudes) satisfying

$$
\left|\overline{\boldsymbol{k}}_{j}\right|=N^{-1}
$$

and $\langle\ldots\rangle_{\overline{\boldsymbol{k}}}$ denotes an average over all possible orientations of the $\overline{\boldsymbol{k}}$ 's.

\section{Exact results}

The geometrical interpretation (B22) leads immediately to some interesting exact results. First, one has the important conclusion that the integral (B21b) vanishes identically if $\bar{\varphi}>1$, for in this case it is impossible to form a closed $(N+1)$-sided planar figure (possibly with crossed lines) with $N$ of the sides constrained by Eq. (B23):

$$
\bar{P}_{0}(\bar{\varphi}) \equiv 0 \quad(\bar{\varphi}>1) .
$$

Next, for $N=1$ and $N=2$ formula (B21b) can be computed exactly. For $N=1$ it is clear from the geometrical interpretation that the integral vanishes unless $|\bar{\varphi}|=1$; since the result is independent of orientation one concludes that

$$
\bar{P}_{0}(\bar{\varphi} ; 1)=\delta(\bar{\varphi}-1)
$$

Consistently, this is just the joint PDF for a pair of random variables $\psi_{1} \doteq \cos \theta, \psi_{2} \doteq \sin \theta$ for $\theta$ distributed uniformly on $[0,2 \pi): P\left(\psi_{1}, \psi_{2}\right)=(2 \pi)^{-1} \delta(|\psi|-1)$, where $|\psi| \doteq\left(\psi_{1}^{2}+\psi_{2}^{2}\right)^{1 / 2}$. This is just the situation described by the case $N=1$, where we identify $\theta$ with the position of the single gyrocenter and $\left\{\psi_{1}, \psi_{2}\right\}$ with the real and imaginary parts of the potential.

For $N=2$ the effective wavevectors are constrained to form a triangle of area $A\left(\overline{\boldsymbol{\varphi}}, \overline{\boldsymbol{k}}_{1}, \overline{\boldsymbol{k}}_{2}\right)$, where

$$
A\left(\boldsymbol{k}_{0}, \boldsymbol{k}_{1}, \boldsymbol{k}_{2}\right)=\frac{1}{2}\left|\boldsymbol{k}_{0} \times \boldsymbol{k}_{1}\right| \delta_{\boldsymbol{k}_{0}+\boldsymbol{k}_{1}+\boldsymbol{k}_{2}, 0} .
$$

In the present case one has $k_{1}=k_{2}=\frac{1}{2}$, so from the simple geometry of an isosceles triangle one obtains

$$
A=\frac{1}{4} \bar{\varphi}\left(1-\bar{\varphi}^{2}\right)^{1 / 2}
$$

Since it is well known ${ }^{7}$ that

$$
\left\langle\delta\left(\boldsymbol{k}_{0}+\boldsymbol{k}_{1}+\boldsymbol{k}_{2}\right)\right\rangle_{\boldsymbol{k}_{1}, \boldsymbol{k}_{2}}=\left[(2 \pi)^{2} A\left(\boldsymbol{k}_{0}, \boldsymbol{k}_{1}, \boldsymbol{k}_{2}\right)\right]^{-1},
$$

\footnotetext{
7 The result (B28) is employed frequently in the reduction of wavenumber convolutions arising in the statistical theory of $2 \mathrm{D}$ homogeneous turbulence; see Ref. [8], Appendix A.
} 
one finally obtains

$$
\bar{P}_{0}(\bar{\varphi} ; 2)= \begin{cases}\frac{2}{\pi\left(1-\bar{\varphi}^{2}\right)^{1 / 2}} & (\bar{\varphi} \leq 1) \\ 0 & (\bar{\varphi}>1) .\end{cases}
$$

This result can also be obtained directly from formula (B21b); see GR 6.522.11.

For $N \geq 3$ it does not appear possible to obtain the integral (B21b) in closed form. For modest $N$, numerical evaluation is feasible. It is convenient to drop some numerical factors from Eq. (B21b) and thus to write

$$
P_{0}(\bar{\varphi})=(2 \pi \bar{\varphi})\left(\frac{N}{\pi}\right) I
$$

where

$$
I(\epsilon ; N) \doteq \frac{1}{2} \int_{0}^{\infty} p d p J_{0}(\sqrt{\epsilon} p) J_{0}^{N}(p / \sqrt{N}) .
$$

Here

$$
\epsilon \doteq N \bar{\epsilon}
$$

where

$$
\bar{\epsilon} \doteq \bar{\varphi}^{2}
$$

As we will verify later, this function is normalized such that

$$
I(0 ; \infty)=1
$$

Because of the rapid oscillations of $J_{0}(\sqrt{\epsilon} p)$ for $\epsilon \gg 1$, straightforward adaptive numerical methods based on grid refinement fail. Therefore, following the guidance in the documentation for the NAG Fortran 77 Library [42], we first evaluate the partial integrals $I_{s}$ defined by integrating the integrand of Eq. (B31) between $j_{0, s}$ and $j_{0, s+1}$, where $j_{0, s}$ is the sth zero of $J_{0}(\sqrt{\epsilon} p)$ (except that $\left.j_{0,0}=0\right)$. Then we consider the sequence $\left\{S_{n}\right\}$ of partial sums $S_{n} \doteq \sum_{s=0}^{n} I_{s}$ and accelerate the convergence of that sequence by means of Shanks' transformation. The results for $N=2$ through $N=7$ are shown in Fig. 24.

The results for $N=6$ and $N=7$ suggest the approach to a limiting function (at least for $\bar{\epsilon} \ll 1$ ), which we will show is $e^{-\epsilon}$. To address the case of large $N$ (the usual case in practice) a variety of asymptotic methods may be employed. We turn to those in the next appendix.

\section{APPENDIX C: ASYMPTOTICS OF $P_{0}$}

We now develop in considerable detail various asymptotic analyses of the fundamental PDF $\mathcal{P}_{0}$. In addition to the central importance of $\mathcal{P}_{0}$ to the Monte Carlo procedure, the asymptotic analysis is interesting in its own right, and comparison of a variety of approaches provides important cross checks on the calculations.

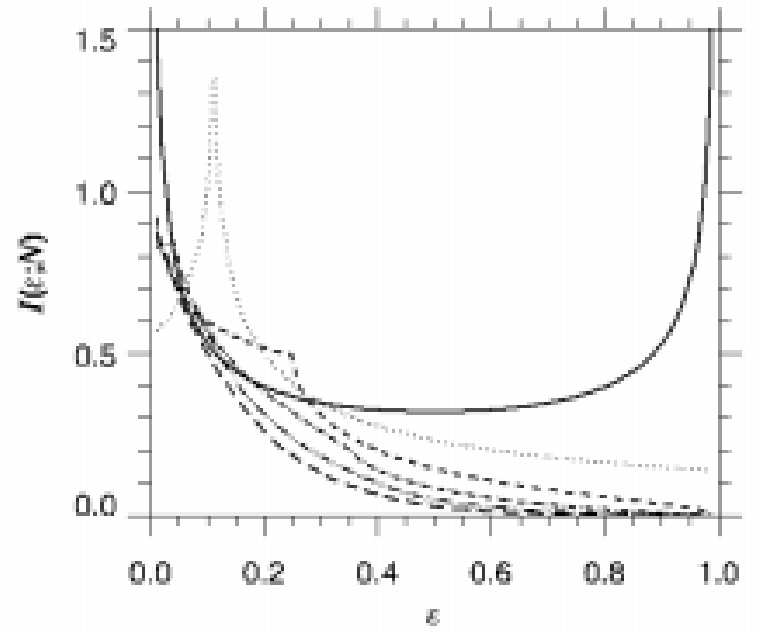

FIG. 24: Numerical evaluation of $\bar{I}(\bar{\epsilon} ; N)$. Solid line, $N=2$; dotted line, $N=3$; short dashed line, $N=4$; dash-dotted line, $N=5$; dash-triple-dotted line, $N=6$; long dashed line, $N=7$. Each curve contains 200 line segments.

\section{The limit $N \rightarrow \infty$}

It is simplest to begin by considering the limit $N \rightarrow \infty$. Note that

$$
\lim _{N \rightarrow \infty} J_{0}^{N}(p / \sqrt{N})=\exp \left(-\frac{1}{4} p^{2}\right) .
$$

(This can be understood as a consequence of the central limit theorem or can be proven directly.) Then

$$
I(\epsilon ; \infty)=\frac{1}{2} \int_{0}^{\infty} p d p J_{0}(\sqrt{\epsilon} p) \exp \left(-\frac{1}{4} p^{2}\right),
$$

which is a standard form (AS 11.4.29):

$$
I(\epsilon ; \infty)=e^{-\epsilon}
$$

The normalization (B34) follows from this as a special case.

The result (C3) is not in obvious agreement with the exact result that $P_{0}=0$ for $\bar{\varphi}>1(\epsilon>N)$. However, upon rewriting Eq. (C3) in terms of $P_{0}=2 \bar{\varphi} N I$, one has

$$
\begin{aligned}
\lim _{N \rightarrow \infty} P_{0}(\bar{\varphi}) & =\lim _{N \rightarrow \infty} 2 \bar{\varphi} N e^{-N \bar{\epsilon}} \\
& =4 \bar{\varphi} \delta(\bar{\epsilon}) \\
& =2 \delta(\bar{\varphi})
\end{aligned}
$$

[consistently normalized as $\left.\int_{0}^{\infty} d \bar{\varphi} P_{0}(\bar{\varphi})=1\right]$.

Since $\epsilon=N \bar{\epsilon}$, the result (C3) suggests plotting $J(\epsilon ; N) \doteq-\ln I / N$. That is done in Fig. 25 for $N=5$ to 10. The form (C3) is seen to be a good approximation for $\bar{\epsilon} \lesssim 0.5$. For $\bar{\epsilon} \lesssim 1$, a noticeable departure from Eq. (C3) is seen; the upward curvature with $\lim _{\bar{\epsilon} \rightarrow 1} J=\infty$ is required in order to satisfy $I=0$ for 


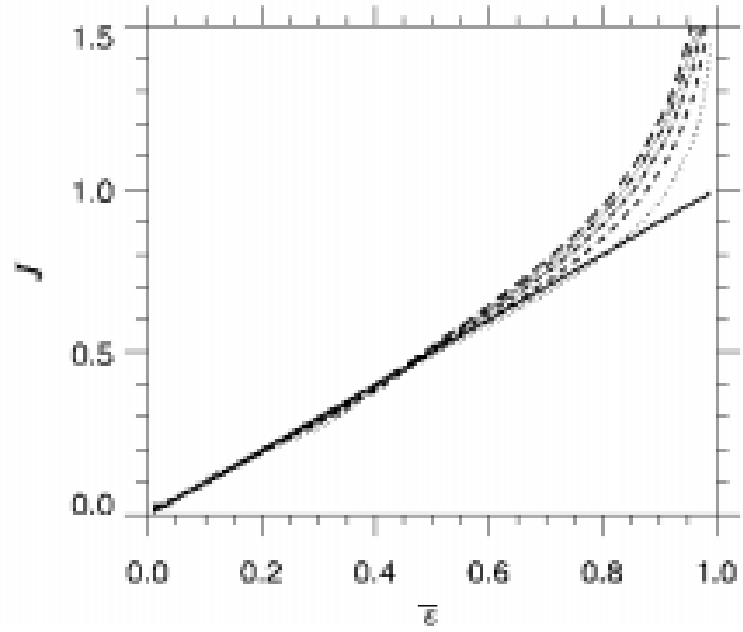

FIG. 25: Numerical evaluation of $J \doteq-\log I / N$. Solid line, reference case $J=\bar{\epsilon}$ [Eq. (C3)]; dotted line, $N=5$; short dashed line, $N=6$; dash-dotted line, $N=7$; dash-tripledotted line, $N=8$; long dashed line, $N=9$.

$\bar{\epsilon}>1$. Except for an overall $N$-dependent height, the curvature near $\bar{\epsilon}=1$ is seen to approach a limiting form. In Sec. C 10 we will use a saddle-point method to reproduce the large $\bar{\epsilon}$ behavior quite well.

\section{Alternate representation of $I(\epsilon ; N)$}

For later use it is convenient to revert to a double integral by recalling that, for any function $F(p)$, where $p \doteq|\boldsymbol{p}|$,

$$
\frac{1}{2 \pi} \int d \boldsymbol{p} e^{i \boldsymbol{p} \cdot \boldsymbol{\varphi}} F(p)=\int_{0}^{\infty} p d p J_{0}(|\varphi| p) F(p)
$$

we identify

$$
\epsilon \doteq|\varphi|^{2}
$$

[Of course, this was from where the form (B31) originally came.] Thus

$$
I(\epsilon ; N)=\frac{1}{4 \pi} \int d \boldsymbol{p} e^{i \boldsymbol{p} \cdot \boldsymbol{\varphi}} J_{0}^{N}(p / \sqrt{N}),
$$

with $I(\epsilon ; \infty)$ following from the use of Eq. (C1).

\section{Cumulant representation}

We now collect some results valid for small $\epsilon$ and finite $N$. First, note that for the evaluation of $I(\epsilon ; \infty)$ one can reproduce Eq. (C3) by replacing

$$
\frac{1}{2} p J_{0}(\sqrt{\epsilon} p) \rightarrow \delta(p-2 \sqrt{\epsilon}) .
$$

This result can be heuristically justified by appealing to the form of Eq. (C7). As we will discuss in much greater detail in Secs. C $9 \mathrm{~b}$ and C 10, the integrand of formula (C7) possesses principal saddle points at height $p=2 \sqrt{\epsilon} \cos \theta$ (exact for $N=\infty$ and approximately true for finite $N$ and small $\epsilon$ ) and $\theta=0$ on the imaginary axes. The role of those saddle points in the value of the final answer is subtle and will be discussed later; certainly their mere existence is not sufficient to rigorously justify Eq. (C8). Nevertheless, the relation $p=2 \sqrt{\epsilon}$ captures the height of the saddle point for small $\epsilon$, and the delta function describes the fact that the integral over the saddle along the contour of steepest descent is $O(1)$. Qualitatively, at least, one might hope that the representation remains useful for finite $N$ as long as $\epsilon$ is sufficiently small. Later we will learn that "sufficiently small" means $\epsilon \ll N$. Assuming this result for the moment, we may estimate the finite- $N$ corrections to Eq. (C3).

If $p=O(\sqrt{\epsilon})$ and $\epsilon \ll N$, then $p=o(\sqrt{N})$. In this limit a cumulant expansion will be rapidly convergent. Define

$$
\widehat{P}(p) \doteq J_{0}^{N}(p / \sqrt{N}) .
$$

By definition, the $l$ th-order cumulants $C_{l}$ are defined by the coefficients in the Taylor expansion of $\ln \widehat{P}$ :

$$
\ln \widehat{P}(p)=\sum_{l=1}^{\infty} \frac{(-i p)^{l}}{l !} C_{l}
$$

or

$$
\widehat{P}(p)=\exp \left(\sum_{l=1}^{\infty} \frac{(-i p)^{l}}{l !} C_{l}\right) .
$$

Because $J_{0}(p / \sqrt{N})$ is real for real $p$, the odd cumulants vanish. Through fourth order, it is straightforward to combine the results

$$
\begin{aligned}
J_{0}(z) & =1-\frac{1}{4} z^{2}+\frac{1}{64} z^{4}+O\left(z^{6}\right), \\
\ln (1+z) & =z-\frac{1}{2} z^{2}+O\left(z^{3}\right),
\end{aligned}
$$

and $\ln J_{0}^{N}=N \ln J_{0}$ to find

$$
\begin{aligned}
C_{2} & =\frac{1}{2}, \\
C_{4} & =-\frac{3}{8} N^{-1}, \\
C_{2 n} & =O\left(N^{-(n-1)}\right),
\end{aligned}
$$

or

$$
\widehat{P}(p)=\exp \left[-\frac{1}{4} p^{2}-\frac{1}{64} N^{-1} p^{4}+O\left(p^{6} / N^{2}\right)\right] .
$$

Upon invoking the delta-function "rule," we estimate that

$$
\begin{aligned}
I(\epsilon ; N) \approx & \int_{0}^{\infty} d p \delta(p-2 \sqrt{\epsilon}) \exp \left[-\frac{1}{4} p^{2}-\frac{1}{64} N^{-1} p^{4}\right. \\
& \left.+O\left(p^{6} / N^{2}\right)\right] \\
= & \exp \left[-\epsilon-\frac{1}{4} N^{-1} \epsilon^{2}+O\left(\epsilon^{3} / N^{2}\right)\right] .
\end{aligned}
$$


The $O\left(\epsilon^{2}\right)$ correction is important for $\epsilon \gtrsim O(\sqrt{N})$. For $\epsilon=O(\sqrt{N})$ the neglected cumulant terms are $O\left(N^{3 / 2} / N^{2}\right)=O\left(N^{-1 / 2}\right)$ and are therefore small for large $N$. They become important for $\epsilon=O(N)$.

A standard form emerges by defining

$$
\begin{aligned}
\bar{\epsilon} & \doteq \epsilon / N=|\bar{\varphi}|^{2}, \\
\bar{I}(\bar{\epsilon} ; N) & \doteq I(\epsilon ; N) .
\end{aligned}
$$

Then our prediction is that

$$
\bar{I}(\bar{\epsilon} ; N) \approx \exp \left[-N\left(\bar{\epsilon}+\frac{1}{4} \bar{\epsilon}^{2}\right)\right] \quad(\bar{\epsilon} \ll 1) .
$$

In fact, Eq. (C16) is not merely qualitatively correct, as the crudeness of the delta-function approximation would suggest; it is quantitatively correct for large $N$. We now proceed to demonstrate this systematically. In Secs. C 4C 6 we consider the regime of small $\bar{\epsilon}$ in detail. Although that material provides motivation, mathematical insights, and consistency checks, it can be skipped on a first reading. A more general asymptotic analysis valid for large $\bar{\epsilon}$ begins in Sec. C 7 .

\section{The truncated cumulant expansion}

We temporarily restrict our attention to the smallamplitude regime $\bar{\epsilon} \ll 1$ and consider the truncated cumulant expansion to be exact:

$$
\begin{aligned}
I(\epsilon ; N) \approx & K(\epsilon ; N) \\
\doteq & \frac{1}{2} \int_{0}^{\infty} p d p J_{0}(\sqrt{\epsilon} p) \\
& \quad \times \exp \left(-\frac{1}{4} p^{2}-\frac{1}{64} N^{-1} p^{4}\right) .
\end{aligned}
$$

Upon changing variables to $t \doteq \frac{1}{4} p^{2}$, one finds the alternate representation

$$
K(\epsilon ; N)=\int_{0}^{\infty} d t J_{0}(2 \sqrt{\epsilon t}) \exp \left(-t-\frac{1}{4} N^{-1} t^{2}\right)
$$

This truncated cumulant expansion is interesting in its own right.

\section{A trick for evaluating $K(\epsilon ; N)$ for $\epsilon=O(\sqrt{N})$}

The form (C18) is amenable to a trick. If one recalls that $\exp \left(-a t^{2}\right)$ behavior results from the Gaussian average of an exponential,

$$
\left(2 \pi \sigma^{2}\right)^{-1 / 2} \int_{-\infty}^{\infty} d \lambda e^{-\lambda^{2} / 2 \sigma^{2}} e^{i t \lambda} \equiv\left\langle e^{i t \lambda}\right\rangle=e^{-\sigma^{2} t^{2} / 2}
$$

one may identify $\sigma^{2}=(2 N)^{-1}$ and write

$$
K(\epsilon ; N)=\left\langle\int_{0}^{\infty} d t J_{0}(2 \sqrt{\epsilon t}) e^{-(1-i \lambda) t}\right\rangle .
$$

The $t$ integral can be performed (AS 11.4.29), and one finds

$$
K(\epsilon ; N)=-\frac{\partial}{\partial \epsilon} \widehat{K}(\epsilon ; N),
$$

where

$$
\begin{aligned}
\widehat{K}(\epsilon ; N) & \doteq\left\langle e^{-\epsilon /(1-i \lambda)}\right\rangle \\
& =e^{-\epsilon}\left\langle\exp \left[-\epsilon\left(\frac{i \lambda-\lambda^{2}}{1+\lambda^{2}}\right)\right]\right\rangle .
\end{aligned}
$$

Evidently the contributions to this integral come predominantly from $\lambda \lesssim O(\sigma)=O\left(N^{-1 / 2}\right)$, so if we consider $\epsilon=O\left(N^{1 / 2}\right)$, then the $\lambda^{2}$ terms in the second exponential are negligible and

$$
\begin{aligned}
\widehat{K}(\epsilon ; N) & \approx e^{-\epsilon}\left\langle e^{-i \epsilon \lambda}\right\rangle \\
& =e^{-\epsilon} e^{-\frac{1}{2} \epsilon^{2} \sigma^{2}} \\
& =\exp \left(-\epsilon-\frac{1}{4} N^{-1} \epsilon^{2}\right)
\end{aligned}
$$

Then from Eq. (C21),

$$
\begin{aligned}
K(\epsilon ; N)= & \left(1+\frac{1}{2} N^{-1} \epsilon\right) \\
& \quad \times \exp \left(-\epsilon-\frac{1}{4} N^{-1} \epsilon^{2}\right) \\
\approx & \exp \left(-\epsilon-\frac{1}{4} N^{-1} \epsilon^{2}\right) .
\end{aligned}
$$

This important result is in exact agreement with the heuristic prediction (C16).

A slight subtlety with this procedure is that if one wants to recover the small- $\epsilon$ behavior correct through terms of $O\left(N^{-1}\right)$, one must retain the $\lambda^{2}$ terms neglected in the previous calculations (although they may be treated as small):

$$
\begin{aligned}
\widehat{K}(\epsilon ; N) & \approx e^{-\epsilon}\left\langle\exp \left[-\epsilon\left(i \lambda-\lambda^{2}\right)\right]\right\rangle \\
& \approx e^{-\epsilon}\left\langle 1-\epsilon\left(i \lambda-\lambda^{2}\right)-\frac{1}{2} \epsilon^{2} \lambda^{2}\right\rangle \\
& =e^{-\epsilon}\left[1+\left(\epsilon-\frac{1}{2} \epsilon^{2}\right) \sigma^{2}\right] \\
K(\epsilon ; N)= & {\left[1-\sigma^{2}\left(1-2 \epsilon+\frac{1}{2} \epsilon^{2}\right)\right] e^{-\epsilon} } \\
= & {\left[1-\frac{1}{2} N^{-1}+\bar{\epsilon}-\frac{1}{4} N \bar{\epsilon}^{2}\right) e^{-N \bar{\epsilon}} } \\
& {\left[\bar{\epsilon}=o\left(N^{-1 / 2}\right)\right] . }
\end{aligned}
$$

\section{Attempts to evaluate $K$ exactly}

We will now describe attempts to evaluate $K$ exactly. Although those will not be wholly successful, they do lead to an asymptotic expansion for the solution in inverse powers of $N$ and to simple results for the small- $\epsilon$ behavior. Although the details are particular to the special form of $K$, for which certain functions can be determined in closed form, and use of the truncated cumulant expansion precludes us from obtaining corrections smaller than 
$O\left(N^{-1}\right)$, the methods are more general and could be applied to $I$ itself if that were deemed important.

We proceed via the Laplace transform

$$
\widehat{K}(z) \doteq \int_{0}^{\infty} d \epsilon e^{-\epsilon z} K(\epsilon)
$$

(We suppress the $N$ dependence temporarily.) It is convenient to use the form (C18). The $\epsilon$ integral is then a standard form (AS 29.3.75):

$$
\begin{aligned}
\widehat{J}(z) & \doteq \int_{0}^{\infty} d \epsilon e^{-\epsilon z} J_{0}(2 \sqrt{\epsilon t}) \\
& =z^{-1} e^{-t / z}
\end{aligned}
$$

Thus

$$
\left.\widehat{K}(z)=z^{-1} \int_{0}^{\infty} d t \exp \left[-\left(1+z^{-1}\right) t-\frac{1}{4} N^{-1} t^{2}\right)\right] .
$$

Define

$$
\beta(z) \doteq \sqrt{N}\left(1+z^{-1}\right)
$$

and recall the definition of the complementary error function:

$$
\operatorname{erfc}(\beta)=\frac{2}{\sqrt{\pi}} \int_{\beta}^{\infty} d \tau e^{-\tau^{2}}
$$

Straightforward algebra leads one to

$$
\widehat{K}(z)=\sqrt{\pi N} z^{-1} e^{\beta^{2}(z)} \operatorname{erfc}(\beta(z)) .
$$

(It is this explicit representation in terms of standard functions that is lacking in the general problem.) The inversion theorem for Laplace transforms then gives

$$
K(\epsilon)=\int_{\mathcal{C}} \frac{d z}{2 \pi i} e^{\epsilon z} \widehat{K}(z),
$$

where $\mathcal{C}$ is a vertical line in the right half of the $z$ plane.

We can use the integral representation (C33) to develop an asymptotic series for $K(\epsilon ; N)$ in inverse powers of $N$. To perform the inversion, it is convenient to integrate over $\beta$ instead of $z$. We first use Cauchy's Theorem to shift $\mathcal{C}$ to the left half-plane, but to the right of $z=-1$. Then

$$
K(\epsilon)=\sqrt{\pi} \int_{\mathcal{C}^{\prime}} \frac{d \beta}{2 \pi i} W(\beta / \sqrt{N}) e^{\beta^{2}} \operatorname{erfc}(\beta),
$$

where

$$
W(\bar{\beta}) \doteq(1-\bar{\beta})^{-1} \exp [-\epsilon /(1-\bar{\beta})] .
$$

If one writes $z=a+i b$, then the contours $\mathcal{C}$ and $\mathcal{C}^{\prime}$ are shown in Figs. 26 and 27 for finite endpoints $b= \pm B$, $-1<a<0$. As $B \rightarrow \infty$ one has an essential singularity of the form $\exp \left[-\epsilon /\left(|a| / B^{2}\right)\right] \rightarrow 0$, so one can close off the contour between points 1 and 5 without changing the value of the integral, thereby obtaining a loop that

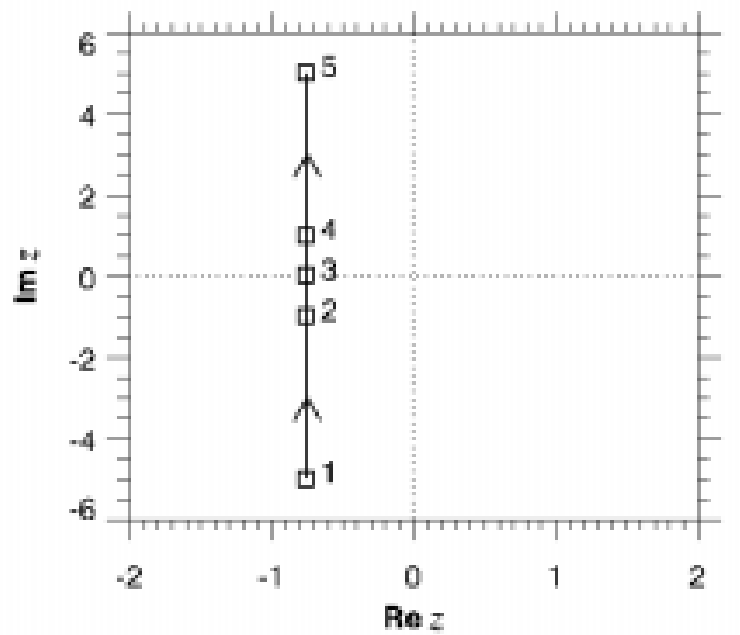

FIG. 26: Integration contour $\mathcal{C}$ for the inverse Laplace transform used in Sec. C6. Points 1-5 are reference values that map to the transformed points in Fig. 27.

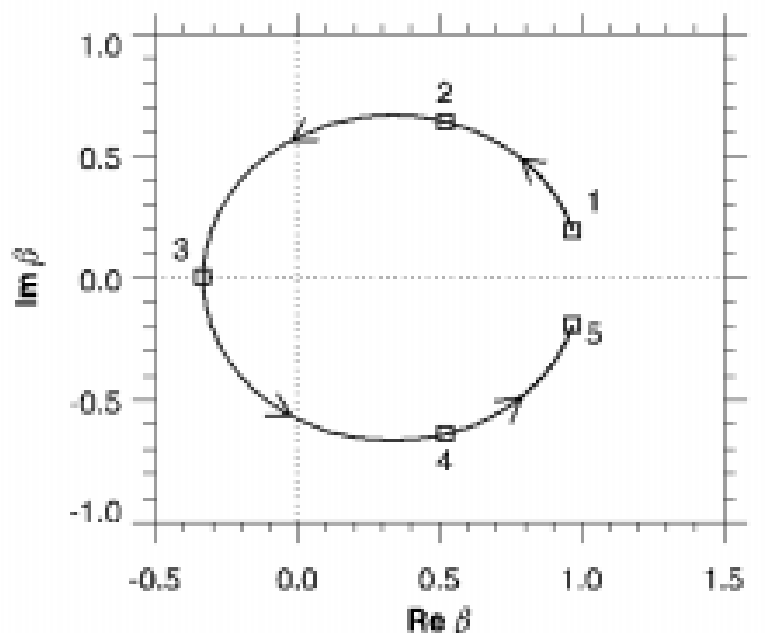

FIG. 27: Transformed integration contour $\mathcal{C}^{\prime}=\beta(\mathcal{C})$. As the endpoints of contour $\mathcal{C}$ (Fig. 26) recede to $\pm i \infty$, points 1 and 5 become coincident.

encircles the origin. Since $\beta$ is large on $\mathcal{C}^{\prime}$, one may use the asymptotic expansion of $e^{\beta^{2}} \operatorname{erfc}(\beta)(\operatorname{AS~7.2.14)~to~}$ find that

$$
\begin{aligned}
K(\epsilon)= & \oint \frac{d \beta}{2 \pi i} W(\beta / \sqrt{N}) \\
& \times \sum_{m=0}^{\infty} \frac{(-1)^{m}(2 m) !}{m ! 2^{2 m} \beta^{2 m+1}} \\
= & \sum_{m=0}^{\infty} \frac{(-1)^{m}}{m !(4 N)^{m}} W^{(2 m)}(0),
\end{aligned}
$$

where the residue theorem was employed in the last step. To simplify the term $W^{(2 m)}(0)$, we use $(1-\bar{\beta})^{-1}=$ 
$\sum_{k=0}^{\infty} \bar{\beta}^{k}$ to arrive at

$$
\begin{aligned}
& W(\bar{\beta})=\exp (\left.-\epsilon \sum_{k=0}^{\infty} \bar{\beta}^{k}\right)\left(\sum_{l=0}^{\infty} \bar{\beta}^{l}\right) \\
&=e^{-\epsilon} \sum_{r=0}^{\infty} \frac{\left(-\epsilon \sum_{k=1}^{\infty} \bar{\beta}^{k}\right)^{r}}{r !} \\
& \times\left(\sum_{l=0}^{\infty} \bar{\beta}^{l}\right) .
\end{aligned}
$$

One has (AS 24.1.2B)

$$
\begin{aligned}
& {\left[\sum_{k=1}^{\infty}\left(\frac{x_{k}}{k}\right) t^{k}\right]^{r}=r ! \sum_{n=r}^{\infty} \frac{t^{n}}{n !} \sum_{\boldsymbol{a}}(n ; \boldsymbol{a})^{*} x_{1}^{a_{1}} \ldots x_{n}^{a_{n}}} \\
& \quad \times \delta\left[\sum_{\bar{n}=1}^{n} \bar{n} a_{\bar{n}}, n\right] \delta\left[\sum_{\bar{n}=1}^{n} a_{\bar{n}}, r\right],
\end{aligned}
$$

where

$$
(n ; \boldsymbol{a})^{*} \doteq \frac{n !}{1^{a_{1}} a_{1} ! 2^{a_{2}} a_{2} ! \ldots n^{a_{n}} a_{n} !},
$$

$a_{i}>0$, and $\delta[a, b]$ denotes the Kronecker delta $\delta_{a, b}$. In the present case one has $x_{k}=k$ and $t=\bar{\beta}$, so one is led to

$$
e^{\epsilon} W(\bar{\beta})=\sum_{r=0}^{\infty}(-\epsilon)^{r} \sum_{l=0}^{\infty} \sum_{n=r}^{\infty} \bar{\beta}^{l+n} \sum_{a} \frac{\delta[\ldots] \delta[\ldots]}{a_{1} ! \ldots a_{n} !} .
$$

Upon differentiating $2 m$ times, one obtains the factor

$$
F \doteq(l+n)(l+n-1) \ldots[l+n-(2 m-1)],
$$

so at $\bar{\beta}=0$ one must have

$$
l+n=2 m, \quad F=(2 m) !,
$$

and $n \leq 2 m$, which corresponds to $l \geq 0$. Thus

$$
e^{\epsilon} W^{(2 m)}(0)=P_{m}(\epsilon),
$$

where

$$
\begin{aligned}
P_{m}(\epsilon) \doteq & (2 m) ! \sum_{r=0}^{2 m}(-\epsilon)^{r} \sum_{n=r}^{2 m} \\
& \times \sum_{a} \frac{\delta\left[\sum \bar{n} a_{\bar{n}}, n\right] \delta\left[\sum a \bar{n}, r\right]}{a_{1} ! \ldots a_{n} !} .
\end{aligned}
$$

Finally, then,

$$
K(\epsilon ; N)=e^{-\epsilon} \sum_{m=0}^{\infty} \frac{(-1)^{m}}{m !(4 N)^{m}} P_{m}(\epsilon) .
$$

As a simple consistency check, observe that if one retains only the $m=0$ term, one again obtains the result (C3) for $K(\epsilon ; \infty)$.
One may exercise the form (C45) in several ways. First, let us consider the series for $K(0 ; N)$. One has

$$
P_{m}(0)=(2 m) ! \sum_{n=0}^{2 m} \sum_{\boldsymbol{a}} \frac{\delta\left[\sum \bar{n} a_{\bar{n}}, n\right] \delta\left[\sum a_{\bar{n}}, 0\right]}{a_{1} ! \ldots a_{n} !} .
$$

However, the constraint $\sum a_{\bar{n}}=0$ implies that every $a$ vanishes, which then means $n=0$ and

$$
P_{m}(0)=(2 m) !,
$$

so

$$
\begin{aligned}
K(0 ; N) & =\sum_{m=0}^{\infty} \frac{(-1)^{m}(2 m) !}{m !(4 N)^{m}} \\
& =1-\frac{1}{2} N^{-1}+\frac{3}{4} N^{-2}-\cdots .
\end{aligned}
$$

This agrees with the alternate derivation

$$
\begin{aligned}
K(0 ; N) & =\lim _{z \rightarrow \infty} z \widehat{K}(z ; N) \\
& =\sqrt{\pi N} e^{N} \operatorname{erfc}(\sqrt{N}),
\end{aligned}
$$

which reduces to Eq. (C48a).

Next, we may compute $K(\epsilon ; N)$ through $O\left(N^{-1}\right)$. It is a straightforward exercise with the form (C44) to determine that

$$
P_{0}(\epsilon)=1, \quad P_{1}(\epsilon)=2-4 \epsilon+\epsilon^{2}
$$

and therefore that

$$
K(\epsilon ; N)=\left[1-\frac{1}{4}\left(2-4 \epsilon+\epsilon^{2}\right) N^{-1}\right] e^{-\epsilon}+O\left(N^{-2}\right) .
$$

This agrees with Eq. (C26b). An alternate approach is to define

$$
K(\epsilon, \alpha) \doteq \int_{0}^{\infty} d t J_{0}(2 \sqrt{\epsilon t}) e^{-\alpha t},
$$

so $K(\epsilon ; \infty)=K(\epsilon, 1)$ and

$$
\begin{aligned}
K(e ; N) \approx & \int_{0}^{\infty} d t J_{0}(2 \sqrt{\epsilon t}) e^{-\alpha t} \\
& \times\left(1-\frac{1}{4} N^{-1} t^{2}\right) \\
= & \left(1-\frac{1}{4 N} \frac{\partial^{2}}{\partial a^{2}}\right) K(\epsilon, \alpha) .
\end{aligned}
$$

This can be reduced straightforwardly to Eq. (C51).

Finally, one may extract from $P_{m}(\epsilon)$ the terms of highest order in $\epsilon$ and sum the entire series (C45). Thus

$$
P_{m}(\epsilon) \rightarrow(2 m) ! \epsilon^{2 m} \sum_{a} \frac{\delta\left[\sum \bar{n} a_{\bar{n}}, 2 m\right] \delta\left[\sum a_{\bar{n}}, 2 m\right]}{a_{1} ! \ldots a_{n} !} .
$$

The constraints are

$$
\sum_{\bar{n}=1}^{2 m} \bar{n} a_{\bar{n}}=2 m, \quad \sum_{\bar{n}=1}^{2 m} a_{\bar{n}}=2 m,
$$


which lead to $\sum_{\bar{n}=2}^{2 m}(\bar{n}-1) a_{\bar{n}}=0$. Therefore one deduces that $a_{\bar{n} \geq 2}=0$ and $a_{1}=2 m$, so $P_{m}(\epsilon) \rightarrow \epsilon^{2 m}$ and

$$
\begin{aligned}
K(\epsilon ; N) & \rightarrow e^{-\epsilon} \sum_{m=0}^{\infty} \frac{(-1)^{m} \epsilon^{2 m}}{m !(4 N)^{m}} \\
& =\exp \left(-\epsilon-\frac{1}{4} N^{-1} \epsilon^{2}\right) .
\end{aligned}
$$

This reproduces the result $(\mathrm{C} 24 \mathrm{~b})$.

By considering the structure of the series for $e^{\epsilon} K$, one can easily deduce that for $\epsilon=O(\sqrt{N})$ the leading-order terms in $\epsilon$ dominate and the previous calculation is correct. For larger $\epsilon$, formerly subdominant terms also contribute. We have been unable to sum the resulting series in closed form, but in any event $K$ is no longer a good approximation to $I$ for such $\epsilon$. Therefore, in the next several sections we return to $I$ and consider approximation techniques appropriate for large $\bar{\epsilon}$ and large $N$.

\section{Standard polar representation for $I$}

We introduce $\bar{\varphi}$ and $q$ by

$$
\varphi \doteq \sqrt{N} \bar{\varphi}, \quad p \doteq \sqrt{N} q
$$

and obtain from Eq. (C7) the standard form

$$
I(\epsilon ; N)=\frac{N}{2 \pi} \operatorname{Re} \int_{-\pi / 2}^{\pi / 2} d \theta \int_{0}^{\infty} q d q \exp [N \Phi(q ; \theta)]
$$

where

$$
\begin{aligned}
\Phi(q ; \theta) & \doteq i \bar{\varphi}_{q}(\theta) q+\ln J_{0}(q) \\
\bar{\varphi}_{q}(\theta) & \doteq \bar{\varphi} \cos \theta
\end{aligned}
$$

(the $q$ subscript on $\bar{\varphi}_{q}$ reminds one that $\bar{\varphi}_{q}$ is the projection of $\boldsymbol{\varphi}$ onto the $\boldsymbol{q}$ vector, not onto a fixed Cartesian $\widehat{\boldsymbol{x}})$. We took advantage of the symmetry in $\theta$ to restrict the integral to $\left(-\frac{1}{2} \pi, \frac{1}{2} \pi\right)$ at the expense of taking the real part. [Because $\bar{\varphi}_{x}(\theta)$ is even, $I$ is also twice the integral from 0 to $\frac{1}{2} \pi$; however, for later use we prefer the symmetrical integration domain.] For $N \rightarrow \infty$, one may replace

$$
\ln J_{0}(q) \rightarrow-\frac{1}{4} q^{2}
$$

\section{The limit $\bar{\epsilon} \geq 1$}

First we will re-establish the remarkable result (B24) that

$$
\bar{I}(\bar{\epsilon} ; N) \equiv 0 \quad(\bar{\epsilon} \geq 1)
$$

Now $\bar{I}$ is proportional to the integral

$$
F(\bar{\varphi} ; \theta)=\int_{0}^{\infty} q d q e^{N \Phi(q, \theta)} .
$$

For real $\theta$ the integrand is analytic everywhere in the finite complex $q$ plane. An application of Cauchy's theorem then leads one to $2 i \operatorname{Im} F=-C$, where $C$ is the integral around a large semicircle in the upper half of the $q$ plane. If the integral vanishes on that semicircle as its radius approaches infinity, one may then deduce that $\operatorname{Im} F(\theta) \equiv 0$ for all $\theta$ between $-\frac{1}{2} \pi$ and $\frac{1}{2} \pi$. Since $F$ is an analytic function of $\theta, F$ itself must vanish. Now for sufficiently large $|q|, J_{0}(q) \sim(2 / \pi q)^{1 / 2} \cos \left(q-\frac{1}{4} \pi\right)$. With $q=x+i y$, one has $|\cos q|=\left(\cos ^{2} x \cosh ^{2} y+\right.$ $\left.\sin ^{2} x \sinh ^{2} y\right)^{1 / 2}$, which can be reduced with the aid of various identities to $|\cos q|=\left(\cosh ^{2} y-\sin ^{2} x\right)^{1 / 2} \leq$ $\cosh |y|$. Convergence is then controlled by

$$
\begin{aligned}
\operatorname{Re}\left[i \bar{\varphi} q+\ln J_{0}(q)\right]= & -\bar{\varphi} y+\ln \left|J_{0}(q)\right| \\
= & -\bar{\varphi} y+\ln \left|\cos \left(q-\frac{1}{4} \pi\right)\right| \\
& \quad+\frac{1}{2} \ln (2 / \pi|q|) \\
& <-\bar{\varphi} y+\ln \cosh y .
\end{aligned}
$$

The least-convergent case is for large $y$, where $\cosh y \sim$ $\frac{1}{2} e^{y} ;$ hence $\operatorname{Re} \varphi<-\bar{\varphi} y+y=(1-\bar{\varphi}) y$. Therefore, for $\bar{\varphi}>1$ convergence is assured and the result (C61) follows. It is easy to see that for sufficiently large $N$ it holds also for $\bar{\varphi}=1$.

\section{Large $N$}

It is noteworthy that the result (C3) is valid for all $\epsilon$ in spite of the rapid oscillations of $J_{0}(\sqrt{\epsilon} p)$ for large $\epsilon$; no asymptotics are involved. This is unfortunate, in a way, because the case of large but finite $N$ is not exactly solvable and some asymptotic methods will be required. It is therefore useful to give an alternate derivation of the result (C3) in order to explain why the rapid oscillations for large $\epsilon$ do not lead to a simplifying asymptotics and to motivate later work. We will show that the result (C3) can be considered to follow from a steepest-descent calculation that is exact for the present case.

We may choose either a Cartesian $\left(p_{x}, p_{y}\right)$ or polar $(p, \theta)$ representation. Each has certain advantages. In general, one expects the polar representation to be superior because it makes explicit the symmetry that the integral depends on only $\varphi \equiv|\varphi|$, not $\varphi_{x}$ and $\varphi_{y}$ separately. However, the $p$ integral runs from 0 to $\infty$, leading to concerns about contributions from the vicinity of the origin. In the Cartesian representation the contours run from $-\infty$ to $\infty$ and the $p_{x}$ and $p_{y}$ integrals behave quite symmetrically; however, that representation obscures the dependence on $\varphi$ alone.

\section{a. Cartesian formulation}

We begin with the Cartesian formulation. Then

$$
I(\epsilon ; \infty)=X\left(\varphi_{x}\right) X\left(\varphi_{y}\right)
$$


where

$$
\begin{aligned}
X\left(\varphi_{x}\right) \doteq & \frac{1}{2 \sqrt{\pi}} \int_{-\infty}^{\infty} d p_{x} e^{i p_{x} \varphi_{x}} \exp \left(-\frac{1}{4} p_{x}^{2}\right) \\
= & \frac{1}{2 \sqrt{\pi}} e^{-\varphi_{x}^{2}} \\
& \quad \times \int_{-\infty}^{\infty} d p_{x} \exp \left[-\frac{1}{4}\left(p_{x}-2 i \varphi_{x}\right)^{2}\right] .
\end{aligned}
$$

Upon introducing $\bar{p}_{x} \doteq p_{x}-2 i \varphi_{x}$, one finds

$$
X\left(\varphi_{x}\right)=\frac{1}{2 \sqrt{\pi}} e^{-\varphi_{x}^{2}} \int_{\overline{\mathcal{C}}} d \bar{p}_{x} \exp \left(-\frac{1}{4} \bar{p}_{x}^{2}\right),
$$

where the contour $\overline{\mathcal{C}}$ is a horizontal line a distance of $2 \varphi_{x}$ below the real axis. However, since the $\bar{p}_{x}$ integral converges within the $90^{\circ}$ cones centered on the real axis, one may use Cauchy's Theorem to deform $\overline{\mathcal{C}}$ to the real axis. The resulting integral, of a Gaussian with variance $\sigma^{2}=2$, is standard; one finds

$$
X\left(\varphi_{x}\right)=e^{-\varphi_{x}^{2}} .
$$

Then

$$
I(\epsilon ; \infty)=e^{-\varphi_{x}^{2}} e^{-\varphi_{y}^{2}}=e^{-|\varphi|^{2}}=e^{-\epsilon} .
$$

The oscillations were transformed away by the contour deformation. That is, the original contour $\mathcal{C}$ may be deformed to the path of steepest descent that passes over the simple saddle centered at $p_{x}=2 i \varphi_{x}$ (see Fig. 28). Although the location of this saddle moves to $i \infty$ as $\varphi_{x} \sim \sqrt{\epsilon} \rightarrow \infty$, the contributions to the integral along the path of steepest descent are sensibly independent of that location, coming from a region of $O(1)$ centered on the saddle.

As is well known, this exact result for integration along a contour of steepest descent over a simple saddle is identical to the result of the standard algorithm that writes

$$
\begin{aligned}
& \int_{\mathcal{C}} d z \exp [N \Phi(z)] \\
& \approx e^{N \Phi\left(z_{0}\right)} \int_{\mathcal{C}} d z \exp \left[\frac{1}{2} N \Phi^{\prime \prime}\left(z_{0}\right)\left(z-z_{0}\right)^{2}\right] \\
& =\left(\frac{2 \pi}{N\left|\Phi^{\prime \prime}\right|}\right)^{1 / 2} e^{N \Phi\left(z_{0}\right)}
\end{aligned}
$$

where $\varphi^{\prime}\left(z_{0}\right)=0$ and we assume for this example that $\Phi^{\prime \prime}\left(z_{0}\right)$ is real and negative. This suggests that in the limit of large but finite $N$ a steepest-descent calculation will still be useful, though no longer exact.

In the limit $N \rightarrow \infty$ one thus observes that the integral factors in a Cartesian representation and may be evaluated by performing two independent contour integrations. Unfortunately, for finite $N$ the presence of higherorder terms $\left[O\left(p^{4}\right)\right]$ in the expansion of $J_{0}^{N}(p / \sqrt{N})$ prevents such a factorization. It is therefore desirable to consider the polar representation in some detail.

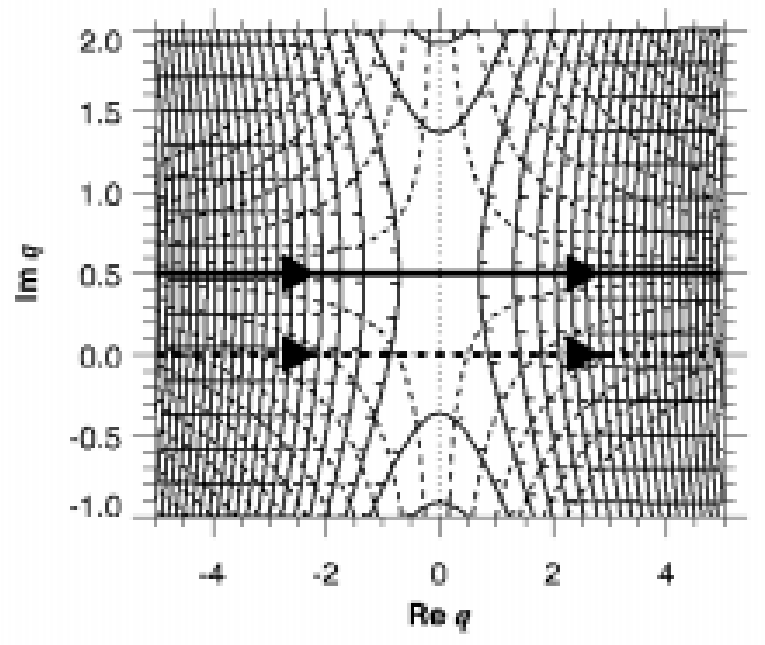

FIG. 28: The simple saddle point for the Cartesian representation with $N=\infty$. Dashed line, original contour; solid line, path of steepest descent. Note that the contour of steepest descent is parallel to the abscissa for a simple quadratic saddle.

\section{b. Polar formulation}

The form of the $q$ integral in Eq. (C58) suggests the use of the method of steepest descent, although we will see that there are complications in the present case because the $q$ integral begins at $q=0$, not $q=-\infty$. Define

$$
I_{ \pm} \doteq \frac{N}{2 \pi} \int_{-\pi / 2}^{\pi / 2} d \theta \int_{0}^{ \pm \infty} q d q e^{N \Phi}
$$

so that $I=\operatorname{Re} I_{+}$. Since the integrand is an analytic function of $q$, one may use Cauchy's theorem to prove that

$$
I_{+}=I_{-}+S,
$$

where $S$ is the contribution from the path $\mathcal{S}$ of steepest descent. A representative contour plot of $\Phi(q)$ is shown in Fig. 29. It reveals the existence of saddle points located near the zeros of $J_{1}(q)$, and of sinks located at the zeros of $J_{0}(q)$. The path of steepest descent is also shown in Fig. 29. Further discussion of the saddle points is given in Sec. C 10.

Unfortunately, by replacing $q \rightarrow-q$ in $I_{-}$and noting that $J_{0}(q)$ is an even function, one can easily prove that $I_{-}=I_{+}^{*}$, so $\operatorname{Re} I_{-}=\operatorname{Re} I_{+}$. Upon taking the real part of Eq. (C71), one therefore proves that $\operatorname{Re} S=0$ and the steepest-descent contribution to $I=\operatorname{Re} I_{+}$vanishes when $q$ is integrated over the entire path $\mathcal{S}$ from $-\infty$ to $\infty$ (and when $\theta$ is integrated over the entire contour running from $-\frac{1}{2} \pi$ to $\frac{1}{2} \pi$ ). Therefore introducing $I_{-}$is not helpful. It is more productive to note that

$$
I_{+}=U+S^{\prime},
$$




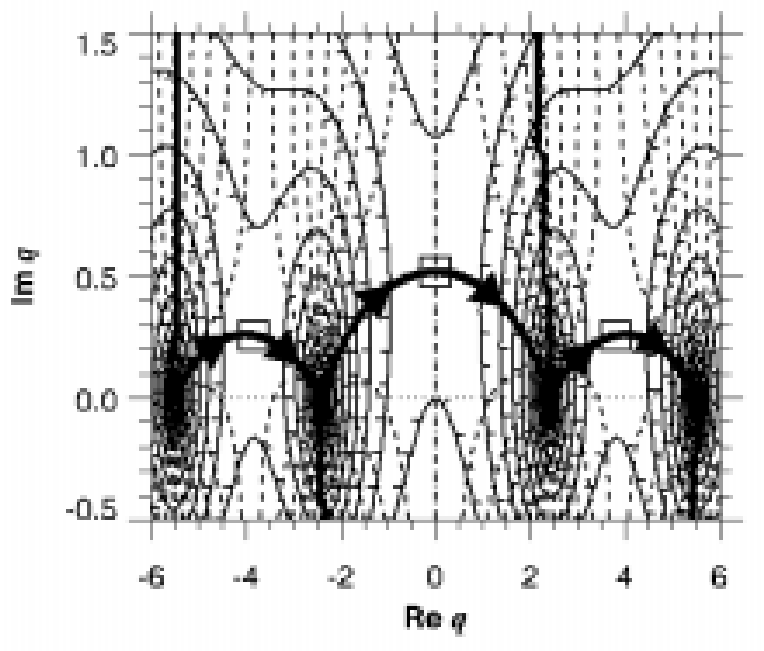

FIG. 29: Contour plot of $\varphi(q)$ for $\bar{\varphi}=0.25$. Saddle points are at the centers of the squares. Solid lines, $\operatorname{Re} \Phi=$ const; dashed lines, $\operatorname{Im} \Phi=$ const. Medium-thickness lines, branch cuts; heavy curve, path of steepest descent.

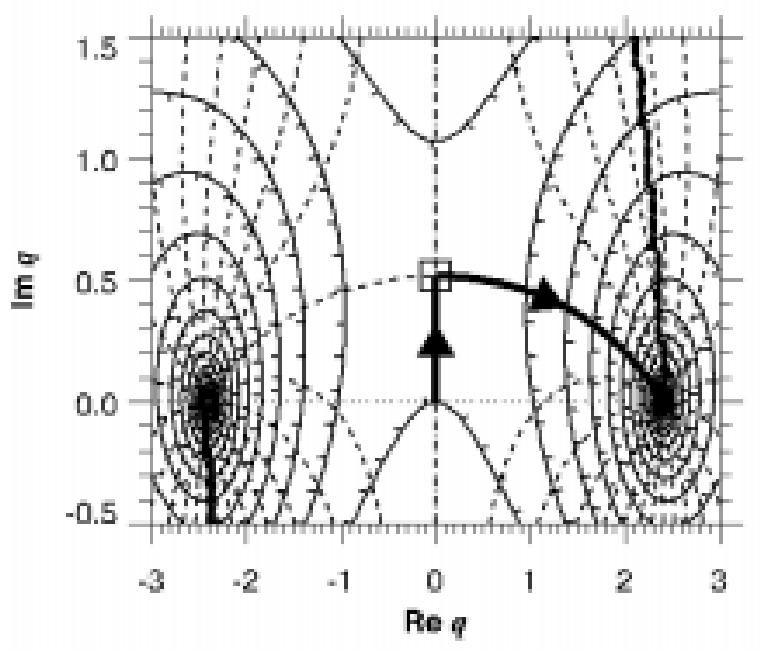

FIG. 30: Alternate integration contour: $\mathcal{U}$ (vertical line) $+\mathcal{S}^{\prime}$ (curved line).

where $U$ is the contribution from the (uphill) path $\mathcal{U}$ along the imaginary axis beginning at $q=0$ and ending at the central saddle point at $q=i \widehat{q}$ ( $\widehat{q}$ being real), and $S^{\prime}$ is the integral over the right-hand half of $\mathcal{S}$ (see Fig. 30). Because the horizontal symmetry has been broken, it is not true that $S^{\prime}$ is purely imaginary; one has

$$
I=\operatorname{Re} U+\operatorname{Re} S^{\prime}
$$

Before attempting a general analysis, it is useful to explicitly verify Eq. (C72) in the special case (C60), for which all integrals can be performed exactly and one can easily understand the sizes of the various contributions.
We thus consider

$$
I_{+}=\frac{N}{2 \pi} \int_{-\pi / 2}^{\pi / 2} d \theta \int_{0}^{\infty} q d q e^{N \Phi},
$$

where

$$
\Phi \doteq i q \bar{\varphi}_{q}-\frac{1}{4} q^{2}
$$

With $q=x+i y$, the path of steepest descent is readily seen to be horizontal, $y=\widehat{q}$ with

$$
\widehat{q}=2 \bar{\varphi}_{q} .
$$

Upon introducing the normalized horizontal distance $p$ from the saddle point by

$$
q=i \widehat{q}+(2 / N)^{1 / 2} p
$$

one has

$$
\begin{aligned}
S^{\prime}= & \frac{1}{\pi} \int_{-\pi / 2}^{\pi / 2} d \theta e^{-\epsilon_{q}} \\
& \times \int_{0}^{\infty} d p\left(p+i \sqrt{2 \epsilon_{q}}\right) e^{-p^{2} / 2} \\
= & S_{r}^{\prime}+i S_{i}^{\prime}
\end{aligned}
$$

where

$$
\begin{aligned}
& S_{r}^{\prime} \doteq \frac{1}{\pi} \int_{-\pi / 2}^{\pi / 2} d \theta e^{-\epsilon_{q}}, \\
& S_{i}^{\prime} \doteq \frac{1}{\sqrt{\pi}} \int_{-\pi / 2}^{\pi / 2} d \theta e^{-\epsilon_{q}} \sqrt{\epsilon_{q}} .
\end{aligned}
$$

Because $\epsilon_{q}=O(N)$ [for $\left.\bar{\varphi}=O(1)\right]$, the imaginary part of $S^{\prime}$ is much larger in magnitude than the real part. Explicitly,

$$
S_{r}^{\prime}=\frac{1}{\pi} \int_{-\pi / 2}^{\pi / 2} d \theta e^{-\epsilon \cos ^{2} \theta}=e^{-\epsilon / 2} I_{0}\left(\frac{1}{2} \epsilon\right)=O\left(\epsilon^{-1 / 2}\right) .
$$

$S_{i}^{\prime}$ is $O(1)$. For both $S_{r}^{\prime}$ and $S_{i}^{\prime}$, note that for large $\epsilon$ the contributions to the integrals come from regions within a distance of order $\sqrt{\epsilon}$ from the end points.

To evaluate $U$, we introduce the normalized vertical distance $p$ from the saddle point by

$$
q=i\left[\widehat{q}+(2 / N)^{1 / 2} p\right],
$$

so that

$$
U=-\frac{1}{\pi} \int_{-\pi / 2}^{\pi / 2} d \theta e^{-\epsilon_{q}} \int_{-\sqrt{2 \epsilon_{q}}}^{0} d p\left(p+\sqrt{2 \epsilon_{q}}\right) e^{p^{2} / 2} .
$$

The $p d p$ integral can readily be done, yielding a contribution

$$
U_{1}=1-S_{r}^{\prime},
$$


where we noted the definition (C79a); the second of these terms cancels the real part of Eq. (C78b). The $\sqrt{\epsilon_{q}}$ term gives a contribution

$$
U_{2}=-\frac{2}{\pi} \int_{0}^{\pi / 2} d \theta e^{-\epsilon \cos ^{2} \theta} \sqrt{2 \epsilon} \cos \theta \int_{0}^{\sqrt{2 \epsilon} \cos \theta} d p e^{p^{2} / 2} .
$$

This can be evaluated exactly by introducing $y \stackrel{\text {. }}{ }$ $\sqrt{2 \epsilon} \sin \theta$. Then

$$
U_{2}=-\frac{2}{\pi} e^{-\epsilon} \int_{0}^{\sqrt{2 \epsilon}} d y e^{y^{2} / 2} \int_{0}^{\sqrt{2 \epsilon-y^{2}}} d p e^{p^{2} / 2} .
$$

The double integral can be interpreted as a 2D Cartesian integral over a quarter circle of radius $\sqrt{2 \epsilon}$, so it is most conveniently done in polar coordinates:

$$
\begin{aligned}
U_{2} & =-\frac{2}{\pi} e^{-\epsilon} \int_{0}^{\pi / 2} d \beta \int_{0}^{\sqrt{2 \epsilon}} \rho d \rho e^{\rho^{2} / 2} \\
& =e^{-\epsilon}-1
\end{aligned}
$$

The -1 cancels against the 1 in Eq. (C83), so

$$
U=e^{-\epsilon}-S_{r}^{\prime}
$$

and, upon adding Eqs. (C78b) and (C87),

$$
I=\operatorname{Re} U+\operatorname{Re} S^{\prime}=e^{-e},
$$

in agreement with Eq. (C68).

Because the dominant term in $S^{\prime}$ is imaginary, so does not contribute to $I$, one might have hoped that $I \approx \operatorname{Re} U$. Unfortunately that is not the case; since according to Eq. (C79a) $S_{r}^{\prime}=O\left(\epsilon^{-1 / 2}\right)$, the second term of Eq. (C87) is much larger than the first, so there is a cancellation of relatively large terms between $S^{\prime}$ and $U$. This poses a problem in the general case (C59), where the position of the saddle point obeys an implicit equation and the $\theta$ integrals that arise cannot be done analytically. Therefore we will consider deformations of the $\theta$ contour and show how to extract the correct answer from Eq. (C79b). Ignore for a moment the constraint that the end points of the $\theta$ integral are pinned to $\pm \frac{1}{2} \pi$. One has explicitly

$$
S_{i}^{\prime}=\left(\frac{\epsilon}{\pi}\right)^{1 / 2} \int_{-\pi / 2}^{\pi / 2} d \theta \cos \theta e^{-\epsilon \cos ^{2} \theta} .
$$

The contour plot of $-\cos ^{2} \theta$ in the complex $\theta$ plane is shown in Fig. 31. Saddle points are found at $\theta= \pm \frac{1}{2} \pi$ and at $\theta=0$. Consider in particular the integral from $q=i \infty$ to $q=-i \infty$, which is along a contour $\mathcal{D}$ of constant phase. With $q=x+i y$ and $\cos \theta=\cos x \cosh y-$ $i \sin x \sinh y$, which reduces to $\cos \theta=\cosh y$ on $\mathcal{D}$, one has

$$
S_{i}^{\prime}=\left(\frac{\epsilon}{\pi}\right)^{1 / 2} \int_{-\infty}^{\infty} d y \cosh y e^{-\epsilon \cosh ^{2} y} .
$$

With $z \doteq \sinh y$ and $\cosh ^{2} y=1+\sinh ^{2} y$, this becomes

$$
S_{i}^{\prime}=\left(\frac{\epsilon}{\pi}\right)^{1 / 2} e^{-\epsilon} \int_{-\infty}^{\infty} d z e^{-\epsilon z^{2}}=e^{-\epsilon} .
$$

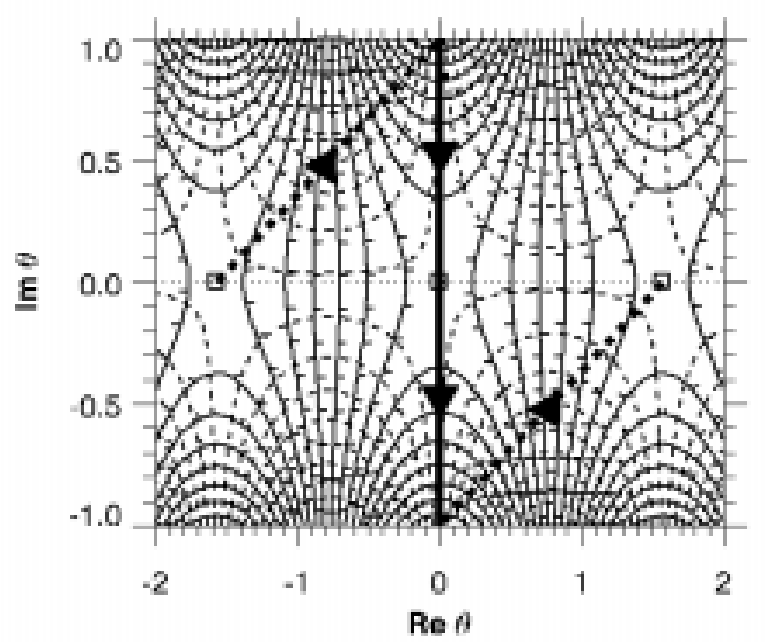

FIG. 31: Contour plot of $-\cos ^{2} \theta$. Dashed lines, contours $\mathcal{C}$; solid line; contour $\mathcal{D}$. Contours $\mathcal{C}$ can be deformed into any curves that connect the indicated endpoints.

Thus one obtains the correct answer by integrating the dominant $q$-saddle-point contribution along the $\theta$ contour $\mathcal{D}$ of steepest descent. Note that although that integral can be done exactly in the present case, it can also be recovered exactly by the standard procedure of Taylor expansion near the saddle point $\theta=0$ and performing a Gaussian integral.

Of course, $S_{i}^{\prime}$ is integrated along the real axis $\mathcal{R}$, not along $\mathcal{D}$. However, since the integrand is analytic, one can write schematically

$$
S_{i}^{\prime}=\int_{\mathcal{R}} d \theta=\int_{\mathcal{C}} d \theta+\int_{\mathcal{D}} d \theta
$$

where $\mathcal{C}$ is an arbitrary contour that connects $\theta=-\frac{1}{2} \pi$ to $\theta=i \infty$ and $\theta=-i \infty$ to $\theta=\frac{1}{2} \pi$; see Fig. 31. Since $S_{i}^{\prime}$ is real by definition, one has $\operatorname{Im} \int_{\mathcal{C}}=-\operatorname{Im} \int_{\mathcal{D}}$. Thus with $\int_{\mathcal{R}} d \theta=\int_{\mathcal{C}} d \theta+\int_{\mathcal{D}} d \theta$, one has

$$
\begin{gathered}
I_{+}=\int_{\mathcal{R}} d \theta \int_{U} d q+\int_{\mathcal{R}} d \theta S^{\prime}, \\
I=\operatorname{Re}(\underbrace{\int_{\mathcal{R}}^{d \theta \int_{U} d q+\int_{\mathcal{R}} d \theta S_{r}^{\prime}}+\underbrace{\int_{\mathcal{C}} d \theta i S_{i}^{\prime}}_{-I})}_{I} \\
+\underbrace{\operatorname{Re} \int_{\mathcal{D}} d \theta i S_{i}^{\prime}}_{I} .
\end{gathered}
$$

We have shown with the underbraces the values of various terms. This procedure of extracting the steepest-descent contribution to the $\theta$ integral can be viewed as adding $0=-I+I$ to the first underbraced terms of Eq. (C93b). However, that interpretation has the deficiency that one could equally well add $\lambda \times 0=-\lambda I+\lambda I$, where $\lambda$ is an arbitrary real number; that would make the numerical 
coefficient of the $\mathcal{D}$ contribution uncertain. That $\lambda=1$ is seen more fundamentally from the decomposition (C93a) (with no real part taken) and by the demonstration that we obtain the correct answer for the special case (C60).

\section{Saddle-point analysis of the limit $\bar{\epsilon} \lesssim 1$}

By appealing to the results for the special $N=\infty$ case treated in the last section, we will in the general case thus approximate

$$
I \approx \frac{1}{2}\left(\frac{N}{2 \pi}\right) \int_{\mathcal{D}} d \theta \int_{\mathcal{S}} q d q e^{N \Phi} .
$$

The factor of $\frac{1}{2}$ takes into account that we are to integrate only over the right-hand half of the $q$ saddle.

For the general structure of the complex $q$ plane, we refer again to Fig. 29. It is easy to verify the existence and orientation of the saddle points for sufficiently small $\bar{\varphi}_{q}$. Stationary points are determined from the solution(s) of

$$
0=\frac{\partial}{\partial q} \Phi(q ; \theta)=i \bar{\varphi}_{q}-\frac{J_{1}(q)}{J_{0}(q)} .
$$

The solutions of this transcendental equation determine functions $\widehat{q}^{(i)} \equiv \widehat{q}^{(i)}(\theta)$, where we label the various roots by $i$; again, the hat signifies dependence on $\theta$. For small $\bar{\varphi}_{x}$, either $J_{1}$ must be relatively small or $J_{0}$ must be relatively large. Since the Bessel functions are bounded according to $\left|J_{\nu}(z)\right| \leq\left|\frac{1}{2} z\right|^{\nu} \exp (|y|) / \Gamma(\nu+1)$ for $\nu \geq-\frac{1}{2}$ (AS 9.1.62) and since for large $z$ the asymptotic forms $J_{\nu}(z)=[2 /(\pi z)]^{1 / 2}\left[\cos \left(z-\frac{1}{2} \nu \pi-\frac{1}{4} \pi\right)+e^{|y|} O\left(|z|^{-1}\right)\right]$ (AS 9.2.1) differ merely by a phase factor, the only possibility is that $J_{1}(q)$ is small, i.e., that $q$ lies near the zeros $j_{1, s}$ of $J_{1}$. Therefore we write

$$
q=j_{1, s}+i \delta \quad(s=0, \pm 1, \pm 2, \ldots)
$$

$\left(j_{1,0}=0\right)$. Then

$$
\begin{aligned}
J_{1}(q) \approx & J_{1}\left(j_{1, s}\right) \\
& \quad+\frac{1}{2}\left[J_{0}\left(j_{1, s}\right)-J_{2}\left(j_{1, s}\right)\right] i \delta \\
= & \frac{1}{2}\left[J_{0}\left(j_{1, s}\right)-J_{2}\left(j_{1, s}\right)\right] i \delta
\end{aligned}
$$

and Eq. (C95) reduces to

$$
\delta=\frac{2 \bar{\varphi}_{q}}{\left[1-J_{2}\left(j_{1, s}\right) / J_{0}\left(j_{1, s}\right)\right]} .
$$

Because the zeros of the Bessel functions interlace, it is always the case that $J_{2}\left(j_{1, s}\right) / J_{0}\left(j_{1, s}\right)<0$, so $\delta$ is always positive. For $s=0$ one has $J_{2}\left(j_{1,0}\right)=J_{2}(0)=0$, so we recover Eq. $(\mathrm{C} 76), q \approx \widehat{q}^{(A)} \approx 2 i \bar{\varphi}_{x}$.

To understand the orientation of these saddle points, we compute

$$
\Phi^{\prime \prime} \equiv \frac{\partial^{2}}{\partial q^{2}} \Phi(q ; \theta)=-R^{\prime}(q)
$$

where

$$
R(q) \doteq J_{1}(q) / J_{0}(q)
$$

With the aid of the identities $J_{0}^{\prime}(z)=-J_{1}(z)$ and $J_{1}^{\prime}(z)=$ $J_{0}(z)-J_{1}(z) / z$, one finds

$$
\begin{aligned}
R^{\prime}(q) & =\frac{J_{0}(q) J_{1}^{\prime}(q)-J_{1}(q) J_{0}^{\prime}(q)}{J_{0}(q)^{2}} \\
& =1-\frac{1}{q} \frac{J_{1}(q)}{J_{0}(q)}+\left(\frac{J_{1}(q)}{J_{0}(q)}\right)^{2} .
\end{aligned}
$$

For the saddles, one may simplify Eq. (C101b) with the aid of Eq. (C95) to

$$
\Phi_{s}^{\prime \prime}=-\left[1-\bar{\varphi}^{2}+\left(\frac{y \bar{\varphi}}{|q|^{2}}\right)-i\left(\frac{x \bar{\varphi}}{|q|^{2}}\right)\right] .
$$

Since $\bar{\varphi}<1$ and $y>0$, one has $\operatorname{Re} \Phi_{s}^{\prime \prime}<0$. For the principal saddle at $\widehat{q}^{(A)}(x=0)$, the path of steepest descent is horizontal. The other saddles are somewhat skewed, but the path of steepest descent is still basically from left to right.

To determine which saddle point dominates, one may formally carry out the saddle-point integrations. One can verify that the principal root $\widehat{q}_{x}^{(A)}$ dominates for small $\bar{\varphi}_{x}$. One can also determine that this root continues to dominate as $\bar{\varphi} \rightarrow 1$.

For finite $\bar{\varphi}$ the principal root must be determined by solution of the transcendental equation (C95). Symmetry guarantees that $\widehat{q}_{x}^{(A)}$ is purely imaginary for all $\bar{\varphi}$, so we introduce the real number $\bar{q}$ by $q=i \bar{q}$; thus one finds the implicit equation for $\bar{q}(\theta)$ to be

$$
I_{1}(\widehat{q}) / I_{0}(\widehat{q})=\bar{\varphi}_{q}(\theta),
$$

where $I_{\nu}$ is the modified Bessel function of the first kind and $\widehat{q}$ is the specific $\bar{q}$ that solves Eq. (C103). [See Fig. 32 for a graphical representation of the solution of Eq. (C103).] Equation (C101b) becomes

$$
R^{\prime}(q) \equiv S(\bar{q})=1-\frac{I_{1}(\bar{q})}{\bar{q} I_{0}(\bar{q})}-\left(\frac{I_{1}(\bar{q})}{I_{0}(\bar{q})}\right)^{2} .
$$

This function has the properties

$$
\begin{aligned}
S(0) & =1 / 2, \\
S(\infty) & =0, \\
S(\bar{q}) & \geq 0 ;
\end{aligned}
$$

it is graphed in Fig. 33. One may rewrite Eq. (C104) in a form more efficient for numerical computation by using Eq. (C103):

$$
S(\widehat{q}(\theta))=1-\bar{\eta}(\widehat{q}(\theta))-\bar{\varphi}_{q}^{2}(\theta),
$$

where

$$
\bar{\eta}(\bar{q}(\theta))=\bar{\varphi}_{q}(\theta) / \bar{q}(\theta)
$$




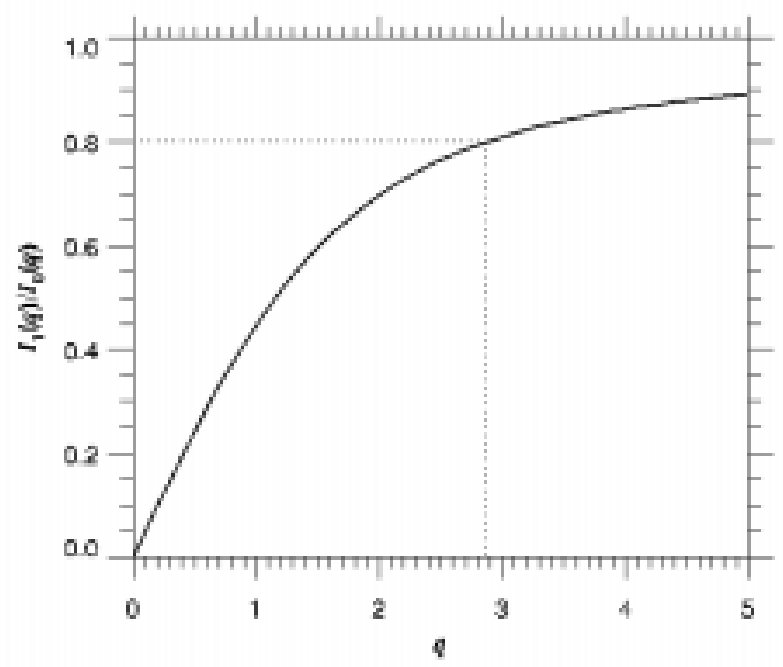

FIG. 32: Illustration of the solution of the implicit equation (C103). Solid curve, $I_{1}(\bar{q}) / I_{0}(\bar{q})$; horizontal dotted line, specified $\bar{\varphi}_{q}$; vertical dotted line, derived $\widehat{q}$. As $\bar{\varphi} \rightarrow 1, \widehat{q} \rightarrow \infty$.

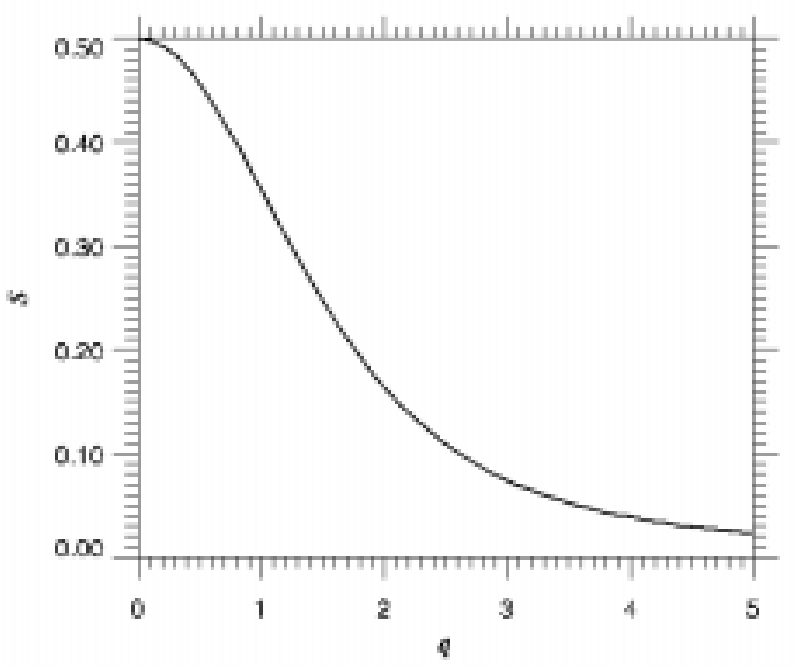

FIG. 33: The function $S(\bar{q})$ [see Eq. (C104)].

We now proceed to analyze the $\theta$ integral. Upon integrating over the principal $q$ saddle using the standard Gaussian approximation, we find

$$
\frac{1}{2} \mathcal{S} \approx \frac{N}{4 \pi} \int_{\mathcal{D}} d \theta i \widehat{q}(\theta) \exp [N \Psi(\theta)]\left(\frac{2 \pi}{N\left|\Phi^{\prime \prime}\right|}\right)^{1 / 2}
$$

where

$$
\Psi(\theta) \doteq-\bar{\varphi}_{q}(\theta) \widehat{q}(\theta)+\ln I_{0}(\widehat{q}(\theta)) .
$$

To find the stationary points, we compute

$$
\begin{aligned}
\Psi^{\prime}(\theta)= & {\left[-\bar{\varphi}_{q}(\theta)+I_{1}(\widehat{q}) / I_{0}(\widehat{q})\right] \widehat{q}(\theta) } \\
& +\bar{\varphi} \widehat{q}(\theta) \sin \theta \\
= & \bar{\varphi} \widehat{q}(\theta) \sin \theta
\end{aligned}
$$

since the term in brackets vanishes by definition of $\widehat{q}$ [see Eq. (C103)]. This result has the same form seen in the special case; we are led again to $\theta=0$ as the principal root. At that point $\bar{\varphi}_{q}=\bar{\varphi}$ and $\Psi^{\prime \prime}(0)=\bar{\varphi} \widehat{q}$, where now $\widehat{q} \equiv \widehat{q}(0)$. Upon completing the $\theta$ integration by integrating vertically downward on $\mathcal{D}$ using the Gaussian approximation, one finally finds

$$
\begin{aligned}
I(\epsilon ; N)= & \frac{1}{2}\left(\frac{1}{\bar{\eta}(\widehat{q}) S(\widehat{q})}\right)^{1 / 2} \\
& \times \exp \left(N\left[-\bar{\varphi} \widehat{q}+\ln I_{0}(\widehat{q})\right]\right) .
\end{aligned}
$$

\section{Summary of the results.}

In summary, for any Fourier amplitude $\varphi_{\boldsymbol{k}} \equiv \varphi$, the natural intensity variable is

$$
\bar{\epsilon} \doteq|\bar{\varphi}|^{2}
$$

[see Eq. (C15a)], where

$$
\bar{\varphi} \doteq\left(1+k^{2}\right) \varphi
$$

[see Eq. (B20)]. The fundamental probability density function $P_{0}(\bar{\varphi})$ is given from Eq. (B30) as $P_{0}(\bar{\varphi})=$ $2 N \bar{\varphi} \bar{I}(\bar{\epsilon} ; N)$. One has the exact result

$$
\bar{I}(\bar{\epsilon} ; N) \equiv 0 \quad(\bar{\epsilon} \geq 1) .
$$

For $\bar{\epsilon}<1$ and $N$ large, one has approximately

$$
\bar{I}(\bar{\epsilon} ; N) \approx\left(\frac{1}{2[\widehat{\eta}(\widehat{q}) S(\widehat{q})]^{1 / 2}}\right) \exp [-N \Psi(\widehat{q})]
$$

(upon introducing a minus sign into the formula to make it look more like a conventional PDF), where

$$
\begin{aligned}
\Psi(\bar{q}) & \doteq \bar{q} \bar{\varphi}-\ln I_{0}(\bar{q}), \\
\bar{\eta}(\bar{q}) & \doteq \bar{\varphi} / \bar{q}, \\
S(\bar{q}) & \doteq 1-\bar{\eta}(\bar{q})-\bar{\varphi}^{2},
\end{aligned}
$$

and $\widehat{q}=\widehat{q}(\bar{\varphi})$ is to be determined by solution of the transcendental equation

$$
I_{1}(\widehat{q}) / I_{0}(\widehat{q})=\bar{\varphi} .
$$

The need to solve Eq. (C117), which is conventionally done by numerical iteration, means that the rigorous numerical evaluation of $\mathcal{P}_{0}$ will be very slow. Fortunately, we generally consider states of intensity sufficiently low that the much simpler approximation $(\mathrm{C} 16)$ is adequate.

Numerical verification of the result (C115) is virtually impossible for realistic $N$ (e.g., $N=1597$ ) because of loss of precision. Nevertheless, even physically very small $N$ may be asymptotically large. In Fig. 34 we compare a direct numerical integration of $J \doteq-N^{-1} \ln \bar{I}$ for $N=17$ with the asymptotic result (C115). The agreement is seen to be virtually perfect; the relative error (for $\bar{I}$ itself, not 


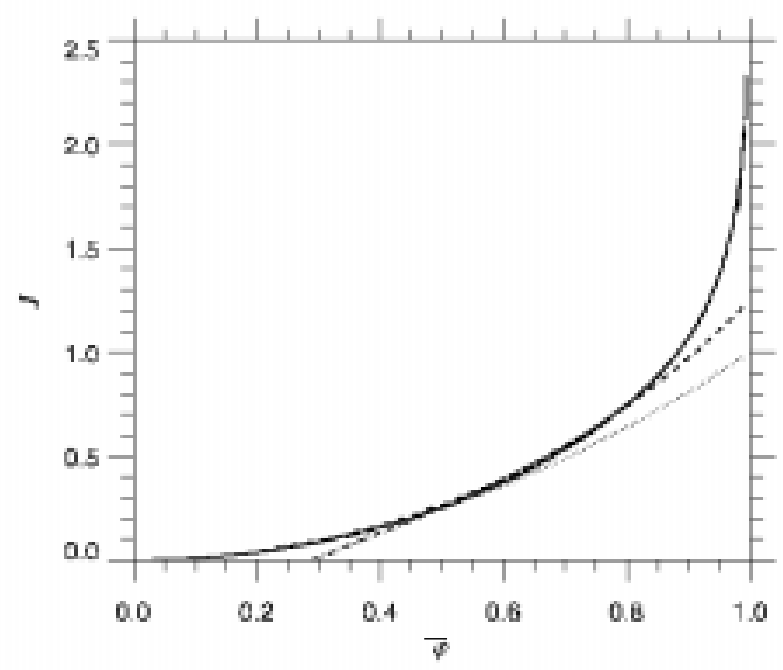

FIG. 34: Comparison of the asymptotic result (C115) with an exact numerical evaluation of $J \doteq-N^{-1} \ln \bar{I}$ for $N=17$. Solid line, exact (numerical) result; long dashed line, (C115) (overlays exact result); dotted line, $y_{1}$ (overlays exact result for $\bar{\varphi} \lesssim 0.6$ ); dashed line, $y_{2}$ (overlays exact result for $\bar{\varphi} \lesssim$ 0.8 ); dash-dotted line, $y_{3}$ (overlays exact result for $\bar{\varphi} \gtrsim 0.45$ ). See the text for the definitions of the approximations $y_{i}$. its logarithm) is less than $0.5 \%$ over the entire domain. Also plotted in these figures are various simpler approximations $y_{i}, i=1,2,3$. The function $y_{1}$ is Eq. (C3), $J \approx \bar{\epsilon} ; y_{2}$ is Eq. (C16), $J \approx \bar{\epsilon}\left(1+\frac{1}{4} \bar{\epsilon}\right)$. The function $y_{3}$ is the result of the asymptotic solution of Eq. (C117) for $x \doteq 1-\bar{\varphi} \ll 1$ :

$$
\begin{gathered}
\bar{q} \approx \\
J \approx\left(\bar{q}+\frac{1}{4}\right) x-\frac{1}{2} \ln (2 \pi \bar{q}) \\
+\frac{1}{2 N} \ln (4 \eta S)
\end{gathered}
$$

This is seen to be an excellent approximation down to about $\bar{\varphi} \approx 0.5$; it is actually used in the Monte Carlo calculations for $\bar{\varphi}$ close to 1 , where the library routine that solves Eq. (C117) has difficulty converging.
[1] D. J. Evans and G. P. Morris, Comput. Phys. Rep. 1, 297 (1984).

[2] W. G. Hoover, Phys. Today 37(1), 44 (1984).

[3] C. K. Birdsall and A. B. Langdon, Plasma Physics via Computer Simulation (McGraw-Hill, New York, 1985).

[4] W. G. Hoover, Computational Statistical Mechanics (Elsevier, New York, 1991).

[5] W. W. Lee, Phys. Fluids 26, 556 (1983).

6] J. A. Krommes, Phys. Plasmas 6, 1477 (1999).

[7] W. W. Lee, Rev. Mod. Phys. (2002), in preparation.

[8] J. A. Krommes, Phys. Rep. 360, 1 (2002).

[9] D. H. E. Dubin, J. A. Krommes, C. R. Oberman, and W. W. Lee, Phys. Fluids 26, 3524 (1983).

[10] R. H. Kraichnan and D. Montgomery, Rep. Prog. Phys. 43, 547 (1980).

[11] A. Hasegawa and K. Mima, Phys. Fluids 21, 87 (1978).

[12] R. H. Kraichnan, Phys. Fluids 10, 1417 (1967).

[13] D. Fyfe and D. Montgomery, Phys. Fluids 22, 246 (1979).

[14] J. Reynders, Ph.D. thesis, Princeton University (1992).

[15] J. A. Krommes, W. W. Lee, and C. Oberman, Phys. Fluids 29, 2421 (1986).

[16] J. A. Krommes, Phys. Rev. Lett. 70, 3067 (1993).

[17] J. A. Krommes, Phys. Fluids B 5, 1066 (1993).

[18] J. Denavit and J. M. Walsh, Comm. Plasma Phys. Control. Fusion 6, 209 (1981).

[19] N. Metropolis, A. Rosenbluth, M. Rosenbluth, A. Teller, and E. Teller, J. Chem. Phys. 21, 108 (1953).

[20] M. E. J. Newman and G. T. Barkema, Monte Carlo Methods in Statistical Physics (Clarendon Press, Oxford, 1999).

[21] R. C. Tolman, The Principles of Statistical Mechanics (Oxford University Press, Oxford, 1938).
[22] P. Terry and W. Horton, Phys. Fluids 25, 491 (1982).

[23] J. C. Bowman, Ph.D. thesis, Princeton University (1992).

[24] R. H. Kraichnan, J. Fluid Mech. 67, 155 (1975).

[25] R. Kubo, Statistical Mechanics (North-Holland, Amsterdam, 1971).

[26] M. H. Kalos and P. A. Whitlock, Monte Carlo Methods, vol. I: Basics (Wiley, New York, 1986).

[27] G. S. Fishman, Monte Carlo: Concepts, Algorithms, and Applications (Springer, New York, 1996).

[28] N. G. van Kampen, Stochastic Processes in Physics and Chemistry (North-Holland, Amsterdam, 1981).

[29] R. D. Rosenkrantz, ed., E. T. Jaynes: Papers on Probability, Statistics, and Statistical Physics (Kluwer Academic Publishers, Dordrecht, 1989).

[30] G. Hu and J. A. Krommes, Phys. Plasmas 1, 863 (1994).

[31] J. A. Krommes and G. Hu, Phys. Plasmas 1, 3211 (1994).

[32] G. Joyce and D. Montgomery, J. Plasma Phys. 10, 107 (1973).

[33] J. B. Taylor and B. McNamara, Phys. Fluids 14, 1492 (1971).

[34] D. A. Schecter, D. H. E. Dubin, K. S. Fine, and C. F. Driscoll, Phys. Fluids 11, 905 (1999).

[35] C. E. Seyler, Jr., Y. Salu, D. Montgomery, and G. Knorr, Phys. Fluids 18, 803 (1975).

[36] C. F. Driscoll, D. Z. Jin, D. A. Schecter, and D. H. E. Dubin, Physica C 369, 21 (2002).

[37] B. Buck and V. A. Macaulay, eds., Maximum Entropy in Action (Clarendon Press, Oxford, 1991).

[38] W. Horton and Y.-H. Ichikawa, Chaos and Structures in Nonlinear Plasmas (World Scientific, Singapore, 1996).

[39] S. Chandrasekhar, Plasma Physics (U. of Chicago Press, Chicago, 1960). 
[40] M. Abramowitz and I. A. Stegun, eds., Handbook of Mathematical Functions (U.S. Government Printing Office, Washington, D.C., 1964).

[41] I. S. Gradshteyn and I. M. Ryzhik, Table of Integrals,
Series, and Products (Academic Press, 1965).

[42] NAG Fortran 77 Library, Mark 19, The Numerical Algorithms Group Ltd, Oxford (1999). 


\section{External Distribution}

Plasma Research Laboratory, Australian National University, Australia

Professor I.R. J ones, Flinders University, Australia

Professor J oão Canalle, Instituto de Fisica DEQ/IF - UERJ , Brazil

Mr. Gerson O. Ludwig, Instituto Nacional de Pesquisas, Brazil

Dr. P.H. Sakanaka, Instituto Fisica, Brazil

The Librarian, Culham Laboratory, England

Library, R61, Rutherford Appleton Laboratory, England

Mrs. S.A. Hutchinson, JET Library, England

Professor M.N. Bussac, Ecole Polytechnique, France

Librarian, Max-Planck-Institut für Plasmaphysik, Germany

J olan Moldvai, Reports Library, MTA KFKI-ATKI, Hungary

Dr. P. Kaw, Institute for Plasma Research, India

Ms. P.J . Pathak, Librarian, Insitute for Plasma Research, India

Ms. Clelia De Palo, Associazione EURATOM-ENEA, I taly

Dr. G. Grosso, Instituto di Fisica del Plasma, Italy

Librarian, Naka Fusion Research Establishment, J AERI, J apan

Library, Plasma Physics Laboratory, Kyoto University, J apan

Research Information Center, National Institute for Fusion Science, J apan

Dr. O. Mitarai, Kyushu Tokai University, J apan

Library, Academia Sinica, Institute of Plasma Physics, People's Republic of China

Shih-Tung Tsai, Institute of Physics, Chinese Academy of Sciences, People's Republic of China

Dr. S. Mirnov, TRINITI, Troitsk, Russian Federation, Russia

Dr. V.S. Strelkov, Kurchatov Institute, Russian Federation, Russia

Professor Peter Lukac, Katedra Fyziky Plazmy MFF UK, Mlynska dolina F-2, Komenskeho Univerzita, SK-842 15 Bratislava, Slovakia

Dr. G.S. Lee, Korea Basic Science Institute, South Korea

Mr. Dennis Bruggink, Fusion Library, University of Wisconsin, USA

Institute for Plasma Research, University of Maryland, USA

Librarian, Fusion Energy Division, Oak Ridge National Laboratory, USA

Librarian, Institute of Fusion Studies, University of Texas, USA

Librarian, Magnetic Fusion Program, Lawrence Livermore National Laboratory, USA

Library, General Atomics, USA

Plasma Physics Group, Fusion Energy Research Program, University of California at San Diego, USA

Plasma Physics Library, Columbia University, USA

Alkesh Punjabi, Center for Fusion Research and Training, Hampton University, USA

Dr. W.M. Stacey, Fusion Research Center, Georgia Institute of Technology, USA

Dr. J ohn Willis, U.S. Department of Energy, Office of Fusion Energy Sciences, USA

Mr. Paul H. Wright, Indianapolis, Indiana, USA 
The Princeton Plasma Physics Laboratory is operated by Princeton University under contract with the U.S. Department of Energy.

\author{
Information Services \\ Princeton Plasma Physics Laboratory \\ P.O. Box 451 \\ Princeton, NJ 08543
}

Phone: 609-243-2750

Fax: 609-243-2751

e-mail: pppl_info@pppl.gov

Internet Address: http://www.pppl.gov 